\title{
INTERPOLATION BY PERIODS IN A PLANAR DOMAIN
}

\author{
M. B. DUBASHINSKIY
}

Dedicated to the memory of Victor Petrovich Havin

\begin{abstract}
Let $\Omega \subset \mathbb{R}^{2}$ be a countably connected domain. With any closed differential form of degree 1 in $\Omega$ with components in $L^{2}(\Omega)$ one associates the sequence of its periods around the holes in $\Omega$, that is around the bounded connected components of $\mathbb{R}^{2} \backslash \Omega$. For which $\Omega$ the collection of such period sequences coincides with $\ell^{2}$ ? We give an answer in terms of metric properties of holes in $\Omega$.
\end{abstract}

\section{INTRODUCTION}

Let $\mathbb{D}$ be the unit disk in the plane $\mathbb{C} \simeq \mathbb{R}^{2}$, and let connected compact sets $B_{1}$, $B_{2}, \cdots \subset \mathbb{D}$ be disjoint. Consider a planar countably connected domain $\Omega:=\mathbb{D} \backslash \bigcup_{j=1}^{\infty} B_{j}$; the sets $B_{1}, B_{2}, \ldots$ are called holes in $\Omega$ (the unbounded connected component of $\mathbb{R}^{2} \backslash \Omega$ will have a special status). Denote by $L_{c}^{2,1}(\Omega)$ the Hilbert space of all closed real differential forms $\omega=\omega_{x} d x+\omega_{y} d y$ of degree 1 defined in $\Omega$ and such that the components $\omega_{x}$ and $\omega_{y}$ are square-integrable over Lebesgue measure in $\Omega$ (one can also deal with vector fields that satisfy analogous conditions). For $\omega \in L_{c}^{2,1}(\Omega)$ and $j=1,2, \ldots$, let $\operatorname{Per}_{j} \omega$ be the period of $\omega$ around the hole $B_{j}$ (see Subsection 1.1 for the details). Next, we define the period operator: put $\operatorname{Per} \omega:=\left\{\operatorname{Per}_{j} \omega\right\}_{j=1}^{\infty}$ for $\omega \in L_{c}^{2,1}(\Omega)$.

We study the following question: for which domains $\Omega$ the set of sequences of the form Per $\omega$ with $\omega \in L_{c}^{2,1}(\Omega)$ coincides with $\ell^{2}$ ? We say that such an $\Omega$ has the complete interpolation property (more precisely, the property of complete interpolation by periods).

The above question is similar to the classical problems on interpolation of a function belonging to some analytic space by its values at prescribed points, see, e.g., [10, 16. Moreover, our problem is equivalent to a similar problem on interpolation of functions in the unweighted Bergman space in $\Omega$ by their complex periods (see Subsection 1.4).

Note that the complete interpolation property of a domain $\Omega$ can be reformulated in the language of homology theory. Namely, the system of curves in $\Omega$ linked to the holes $B_{j}$ should be a Riesz basis (see [2,10]) in the space of $L^{2}$-homologies in $\Omega$ (see the remark at the end of Subsection 2.3).

Note also that the question on interpolation by periods reduces by duality to estimation of functions harmonic in $\Omega$ with locally-constant Dirichlet boundary data (Proposition 2.10). This estimate can be of independent interest.

The main result of this paper is description of the domains $\Omega$ possessing the complete interpolation property in terms of metric characteristics of the mutual layout of sets $B_{j}$, $j \in \mathbb{N}$ (Theorem 5.8).

2010 Mathematics Subject Classification. Primary: 30C85; Secondary: 31A15, 30E05, 30H20, 58A14, 26D15.

Key words and phrases. Infinitely-connected domain, periods of forms, interpolation, Riesz basis, harmonic functions.

Supported by the Russian Science Foundation grant 14-21-00035. 


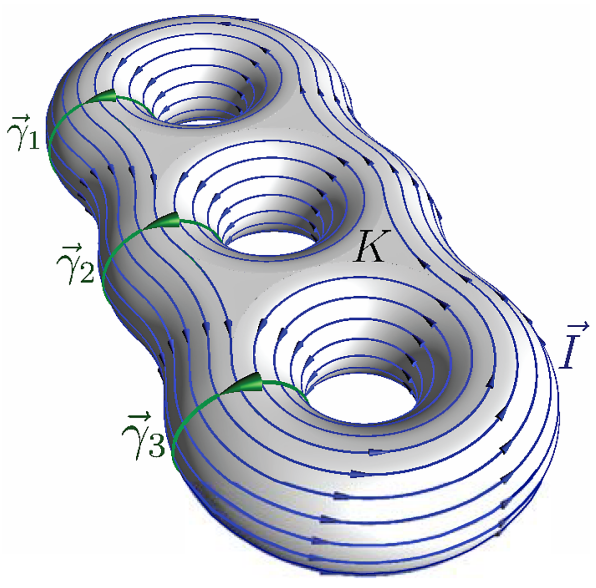

FIGURE 1. Equilibrium current on multiply-connected set.

The most difficult is to derive the first condition of Theorem 5.8 (the uniform local finiteness property) from the complete interpolation property; this is done in Theorem 4.2. Nevertheless, the first three conditions of Theorem 5.8 look naturally from a viewpoint of the classical theory of interpolation in analytic spaces, where, roughly speaking, the corresponding conditions are the Blaschke condition and the separation conditions. The fourth condition of Theorem 5.8 (the capacity connectedness) seems to be most exotic. This condition is the connectedness of a graph that consists of condensers formed by the connected components of $\mathbb{R}^{2} \backslash \Omega$ and having not very small capacity.

Motivation. The interest to the planar problem on interpolation by periods is raised by the following high-dimensional problem on an energy minimizing electric current. In $\mathbb{R}^{3}$, consider a closed domain $K$ bounded by a surface homeomorphic to the sphere with three handles (see Figure 1). Put three closed oriented curves $\gamma_{1}, \gamma_{2}, \gamma_{3}$ supported on the boundary $\partial K$ and bounding oriented surfaces $S_{1}, S_{2}, S_{3}$ (sections) respectively in such a way that the set $K \backslash\left(S_{1} \cup S_{2} \cup S_{3}\right)$ is simply connected. Pick $a_{1}, a_{2}, a_{3} \in \mathbb{R}$. Let us seek an electric current $\vec{I}$ supported on $K$ and such that:

(1) $\operatorname{div} \vec{I}=0$;

(2) $\int_{S_{j}}\langle\vec{I}, \vec{n}\rangle d \mathcal{H}^{2}=a_{j}$ for $j=1,2,3$; here $\vec{n}$ is the unit normal field orienting the surfaces $S_{j}$, and $\mathcal{H}^{2}$ is area measure on these surfaces;

(3) the current $\vec{I}$ gives the minimum to the energy

$$
W(\vec{I}):=\int_{\mathbb{R}^{3}} \int_{\mathbb{R}^{3}} \frac{\langle\vec{I}(x), \vec{I}(y)\rangle}{|x-y|} d x d y .
$$

Such an energy minimizing current $\vec{I}$ is called an equilibrium current. This notion was introduced in [13]. If $W(\vec{I})<+\infty$, then $\vec{I}$ is a distributional vector field (or de Rham current, see [4]) whose components in some coordinate system can be represented as a sum of distributional partial derivatives of $L^{2}$-functions. One can properly define flows of such currents through surfaces $S_{j}$.

If the boundary $\partial K$ is sufficiently smooth, then the equilibrium current $\vec{I}$ can be found by solving the Laplace equation for multivalued function in $\mathbb{R}^{3} \backslash K$. Nevertheless, we wish to establish the theory of equilibrium currents in the same generality in which the classical capacity theory is developed. For this, the problem on equilibrium currents should be stated for arbitrary $K \subset \mathbb{R}^{3}$. The nonregularity of the boundary $\partial K$ can 


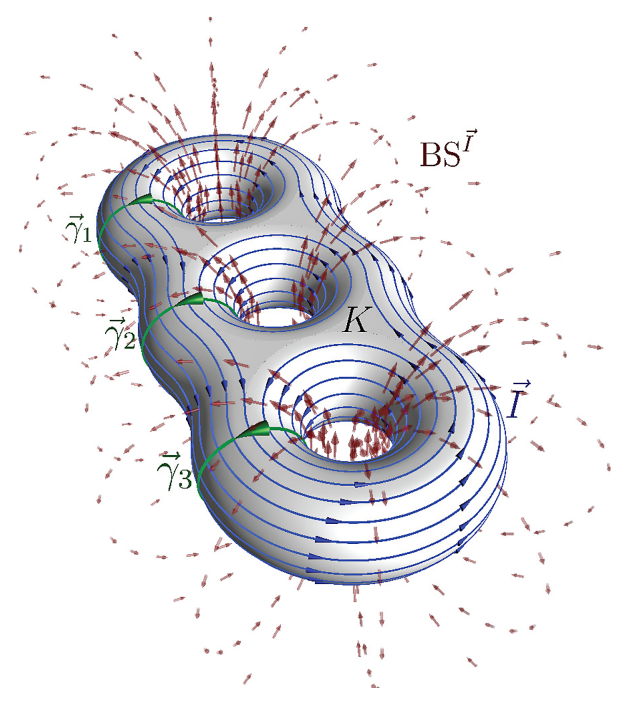

FiguRE 2. Biot-Savart field.

be of several kinds. First, this can be a "differential" nonsmoothness, for example, if there are "cusps" on $\partial K$; a number of publications were devoted to the behavior of solutions of the Laplace operator near such singularities. Another type of nonsmoothness is the topological complexity of $K$. This occurs if $K$ has infinitely many holes, that is if homology spaces of $\mathbb{R}^{3} \backslash K$ are infinite-dimensional. We immediately arrive at the following questions: what should be the appropriate normalizing conditions on the flow of $\vec{I}$ through the sections $S_{j}$ ? to what extent does such a problem depend on the choice of a system of sections? The author does not know anything about this.

One can also ask the following: which compact sets $K \subset \mathbb{R}^{3}$ can support a nonzero current with finite energy? It is easily seen that then $K$ can also support a nonzero scalar charge with finite energy. It can be proved that there exist sets $K \subset \mathbb{R}^{3}$ supporting scalar charges of finite energy but supporting no solenoidal currents with finite energy. One can take an appropriate Cantor-type set for such $K$.

Thus, the solenoidality condition $\operatorname{div} \vec{I}=0$ makes our problem different from the classical question on the sets of positive capacity.

Now we point out the relationship between the problem on an equilibrium current and the problem on interpolation by periods. An electric current $\vec{I}$ generates magnetic Biot-Savart field (see Figure 2) given by formula

$$
\mathrm{BS}^{\vec{I}}=\frac{1}{4 \pi} \operatorname{curl}(\vec{I} \star 1 /|x|) .
$$

Here $\star$ denotes convolution and the operator curl is understood in the sense of distributions. The role of such potentials in approximation theory was studied in [1] and [9].

If $\vec{I}$ is an equilibrium current then the field $\mathrm{BS}^{\vec{I}}$ must vanish in the interior of $K$. Put $\vec{f}=\mathrm{BS}^{\vec{I}}$. One can show that the problem on an equilibrium current stated above is equivalent to the following problem on its magnetic field: to find a field $\vec{f}$ in $\mathbb{R}^{3} \backslash K$ such that

(1) $\operatorname{curl} \vec{f}=0$ in $\mathbb{R}^{3} \backslash K$;

(2) the circulations of $\vec{f}$ over the curves $\gamma_{j}$ have prescribed values $4 \pi a_{j}, j=1,2,3$; 
(3) under the above two conditions the field $\vec{f}$ gives the minimum to the energy norm $\int_{\mathbb{R}^{3} \backslash K}|\vec{f}(x)|^{2} d x$.

Thus, the problem on equilibrium electric current reduces to the problem on interpolation of a vector field (or of a differential form of degree 1) in the exterior domain $\mathbb{R}^{3} \backslash K$ by its periods.

The author does not know anything about such a high-dimensional problem. In this paper we only study its two-dimensional analog. Let us remark that, in accordance with the high-dimensional motivation, we would like to avoid complex variable techniques in the two-dimensional problem. Actually, these methods will not be used indeed, most of the proofs will be obtained by real-valued estimates.

Organization of the paper. In $\$ 1$ we give the main definitions related to the problem of interpolation by periods and state its simplest properties. The complete interpolation property defined above is equivalent to the fact that the following two conditions are satisfied: first, the operator Per: $L_{c}^{2,1}(\Omega) \rightarrow \ell^{2}$ should be bounded (in this case we say that $\Omega$ possesses the Bessel property); second, the operator Per: $L_{c}^{2,1}(\Omega) \rightarrow \ell^{2}$ should be surjective (then we say that $\Omega$ has the interpolation property). The Bessel constant $C_{B}(\Omega)$ is defined as the norm of the operator Per: $L_{c}^{2,1}(\Omega) \rightarrow \ell^{2}$, whereas the interpolation constant $C_{I}(\Omega)$ is the minimal norm of the operator right-inverse to Per. We also use the weak Bessel constant $\widetilde{C}_{B}(\Omega)$, which is the supremum of the norms of the functionals $\operatorname{Per}_{j}: L_{c}^{2,1}(\Omega) \rightarrow \mathbb{R}$ over $j=1,2, \ldots$ The property of complete interpolation by periods means that $C_{I}(\Omega)<+\infty$ and $C_{B}(\Omega)<+\infty$. If $\widetilde{C}_{B}(\Omega)<+\infty$, then we say that $\Omega$ has the weak Bessel property.

The problem on interpolation by periods turns out to be conformally invariant. Also, the Bessel constant, the weak Bessel constant, and the interpolation constant change in a controlled way under application of a quasiconformal diffeomorphism to $\Omega$ (Proposition 1.3).

In 92 we prove the basic results on interpolation by periods. In Subsection 2.2 we find the reproducing kernels of the functionals $\operatorname{Per}_{j}$, i.e., forms $\kappa_{j} \in L_{c}^{2,1}(\Omega)$ such that $\operatorname{Per}_{j} \omega=\left\langle\omega, \kappa_{j}\right\rangle_{L_{c}^{2,1}(\Omega)}$ for any $\omega \in L_{c}^{2,1}(\Omega)$. To find these $\kappa_{j}$, one should solve the Dirichlet problem for the Laplace operator in the domain $\Omega$ with locally-constant boundary data, see Theorem [2.4. It turns out (Proposition 2.6) that $\left\langle\kappa_{j}, \kappa_{j^{\prime}}\right\rangle<0$ for any different $j, j^{\prime} \in \mathbb{N}$. This immediately implies (Theorem 2.16) that if $\widetilde{C}_{B}(\Omega)<+\infty$ then $C_{B}(\Omega)<+\infty$; in other words, the weak Bessel property of the domain $\Omega$ implies its Bessel property. The Bessel property of $\Omega$ is thus equivalent to the estimate $\sup _{j \in \mathbb{N}} \operatorname{Cap}_{2}\left(B_{j}, \mathbb{R}^{2} \backslash\left(\Omega \cup B_{j}\right)\right)<+\infty$; here $\operatorname{Cap}_{2}(\cdot, \cdot)$ is the capacity of a condenser with two plates, see Subsection 2.5. It seems that there exist no simpler metric conditions on the holes $B_{j}$ that describe the Bessel property without additional conditions on interpolation or on the uniform local finiteness property, which we define below.

The complete interpolation property means that the system $\left\{\kappa_{j}\right\}_{j=1}^{\infty}$ is a Riesz basis in its closed linear span. In Subsection 2.4 we interpret this condition taking into account the explicit form of reproducing kernels. For given $a_{1}, a_{2}, \cdots \in \mathbb{R}$, consider a function $u: \mathbb{R}^{2} \rightarrow \mathbb{R}$ for which $\Delta u=0$ in $\Omega, u=a_{j}$ on $B_{j}$ for $j=1,2, \ldots$, and $u=0$ in $\mathbb{D}^{(c)}=\mathbb{R}^{2} \backslash \mathbb{D}$. It turns out (Proposition 2.10) that the complete interpolation property is equivalent to the estimate

$$
C_{I}^{-2}(\Omega) \cdot \sum_{j=1}^{\infty} a_{j}^{2} \leq \int_{\mathbb{R}^{2}}|\nabla u|^{2} d \lambda_{2} \leq C_{B}^{2}(\Omega) \cdot \sum_{j=1}^{\infty} a_{j}^{2}
$$


for any $a_{1}, a_{2}, \cdots \in \mathbb{R}$; here $\lambda_{2}$ is the two-dimensional Lebesgue measure in $\mathbb{R}^{2}$. Thus, estimates on closed forms are reduced to estimates on harmonic functions. Since harmonic functions minimize the Dirichlet integral under given boundary Dirichlet data, it is convenient to work with the class of Sobolev functions locally constant in $\mathbb{R}^{2} \backslash \Omega$ and equal to zero in $\mathbb{D}^{(c)}$. We say that such functions are admissible (for the domain $\Omega$ ), denoting the space of admissible functions by $\mathcal{A} d m(\Omega)$.

Our main goal in 3 is to derive a partial criterion of the complete interpolation property for the domain $\Omega$. Namely, necessary and sufficient conditions will be obtained in the case where one of the following assumptions is satisfied: either

$$
\inf _{j \in \mathbb{N}} \operatorname{diam}_{H}\left(B_{j}\right)>0
$$

$\left(\operatorname{diam}_{H}\right.$ is the hyperbolic diameter in $\left.\mathbb{D}\right)$, or

$(\diamond \diamond) \exists \varepsilon>0: \operatorname{dist}\left(B_{j}, B_{j^{\prime}}\right) \geq \varepsilon \cdot \max \left\{\operatorname{diam} B_{j}, \operatorname{diam} B_{j^{\prime}}\right\}$ for any different $j, j^{\prime} \in \mathbb{N}$.

The last condition is called the strong separation of the holes $B_{j}$. Theorem 3.7 states

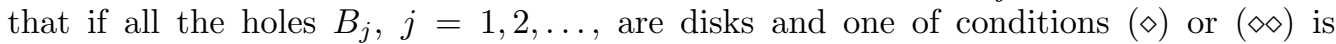
fulfilled, then the complete interpolation property of the domain $\Omega$ is equivalent to the two conditions $(\underline{\nabla})$ and $(\vec{\Delta})$. At the same time, none of conditions $(\vec{\Delta})$ and $(\vec{\nabla})$ is necessary for the complete interpolation property of $\Omega$ (see Example 3.3 in Subsection 3.3 ). Here we use the patch method in order to construct admissible functions. Also, an analog of Carleson measures arises in proofs (Subsection 3.1).

Let $\mathcal{B}\left(0, \frac{1}{2}\right) \subset \mathbb{D}$ be a disk centered at the origin and of radius $\frac{1}{2}$. The examples in Subsection 3.3 lead to the following question: is it possible to construct domains $\Omega$ with $C_{I}(\Omega)$ and $C_{B}(\Omega)$ bounded from above and such that the disk $\mathcal{B}\left(0, \frac{1}{2}\right)$ intersects a very large number of holes $B_{j}$ ? The answer is in the negative. Let us say that a domain $\Omega$ has the uniform local finiteness property if there exists $N=N(\Omega)<+\infty$ such that any disk in the hyperbolic metric in $\mathbb{D}$ of hyperbolic diameter 1 intersects at most $N$ holes $B_{j}$. Theorem 4.2 in $\$ 4$ is the crucial point of our study. It states that the complete interpolation property of $\Omega$ implies its uniform local finiteness property. In the proof, taking only the estimate $\sup _{j \in \mathbb{N}} \operatorname{Cap}_{2}\left(B_{j}, \mathbb{R}^{2} \backslash\left(\Omega \cup B_{j}\right)\right)<+\infty$ into account, we construct a function $u \in \mathcal{A} d m(\Omega)$ for which the values of $u$ on the holes $B_{j}$ are not too small, but the integral $\int_{\mathbb{R}^{2}}|\nabla u|^{2} d \lambda_{2}$ is not very large. Such a function is obtained as a distance to $\mathbb{D}^{(c)}$ in the inner metric generated by the conformal metric $\mathbb{1}_{\Omega}|d z|$. Lower estimates for $\left.u\right|_{B_{j}}$ are derived from capacity estimates by subdividing geodesics in the above-mentioned metric into appropriate arcs (see Lemmas 4.3, 4.4, and 4.5).

Under the uniform local finiteness condition, it is possible to give simple metric criteria for the Bessel property and for the interpolation property of $\Omega$ (separately). So, under the uniform local finiteness condition, the Bessel property turns out to be equivalent to the condition of the weak separation of the holes $B_{j}: \operatorname{dist}\left(B_{j}, B_{j^{\prime}}\right) \geq$ $\varepsilon \cdot \min \left\{\operatorname{diam} B_{j}, \operatorname{diam} B_{j^{\prime}}\right\}$ for any $j, j^{\prime}=1,2, \ldots, j \neq j^{\prime}$, and some $\varepsilon>0$ not depending on $j$ and $j^{\prime}$. This fact is proved by using the equivalence of the Bessel and weak Bessel properties (Theorem 5.1). We give also a metric proof of this criterion, which does not use abstract estimates of reproducing kernels but is based on the existence of sufficiently wide annular regions in $\Omega$. In this proof, a partial order structure on the set of holes arises naturally: roughly speaking, $B_{j} \succ B_{k}$ means that $\operatorname{diam} B_{k} \ll \operatorname{diam} B_{j}$ and $\operatorname{dist}\left(B_{j}, B_{k}\right) \ll \operatorname{diam} B_{j}$. From our viewpoint, the existence of such a structure is the most precise metric expression of the continuity of the operator Per.

The interpolation property of $\Omega$ turns out to be equivalent to the above-mentioned capacity connectedness (see the definitions of the graphs $G(\Omega, S)$ and $g(\Omega, s)$ at the beginning of Subsection 5.2 ) whenever the family of holes in $\Omega$ is uniformly locally finite. 
This is proved in Theorems 5.4 and 5.5 in Subsection 5.2 In the proofs we implement the connectedness of $G(\Omega, S)$ by constructing in $\mathbb{R}^{2}$ planar "roads" that correspond to the edges of $G(\Omega, S)$ and connect holes $B_{j}$. The interpolation property turns out to be nonmonotone with respect to the set of holes in the following sense: this property may fail if we erase some holes in $\Omega$ (see the remark after Theorem 5.5).

Until Subsection 6.2 we assume that every hole $B_{j}$ is a closure of a domain with a sufficiently smooth boundary. In Subsection 6.2, we generalize the criterion of complete interpolation (Theorem 5.8) to the case of arbitrary holes $B_{j}$, assuming only that any $B_{j}$ is a continuum not separating the plane. For this, we approximate such $B_{j}$ 's by sets with smooth boundaries.

Finally, in Subsection 6.3 we formulate several open questions generalizing the planar problem on interpolation by periods. In particular, one of such generalizations delivers a nontrivial quasiconformal invariant of countably-connected Riemann surfaces - the existence of an integral Riesz basis in the $L^{2}-($ co)homology space.

Some technical proofs skipped in the main exposition are presented in the Appendix (Section A).

All our estimates are constructive in the following sense: if we prove the finiteness of some quantity, then our arguments allow us to obtain an explicit estimate on this quantity.

Some notation. The complex plane $\mathbb{C}$ is always identified with $\mathbb{R}^{2}$. The symbol $\mathbb{D}$ denotes the open unit disk in $\mathbb{C}: \mathbb{D}=\{z \in \mathbb{C}:|z|<1\}$. If $E \subset \mathbb{C}$, then $E^{(c)}$ denotes $\mathbb{C} \backslash E$. Thus, $\mathbb{D}^{(c)}=\{z \in \mathbb{C}:|z| \geq 1\}$.

The symbol $\lambda_{d}$ denotes Lebesgue measure in $\mathbb{R}^{d}$, usually $d=2$, sometimes $d=1$. "Almost everywhere" or "a.e." means "almost everywhere with respect to the twodimensional Lebesgue measure $\lambda_{2}$ ". The measure $\mathcal{H}^{1}$ is the one-dimensional Hausdorff measure, i.e., the length measure on various curves.

The Euclidean distance on the plane $\mathbb{C}=\mathbb{R}^{2}$ is denoted by dist, whereas $\operatorname{dist}_{H}$ stands for the distance in the hyperbolic metric $\frac{2|d z|}{(1-|z|)^{2}}$ in the unit disk $\mathbb{D}$. We denote by $\operatorname{diam} E$ and $\operatorname{diam}_{H}(E)$ the Euclidean and hyperbolic diameters of a set $E \subset \mathbb{C}$ or $E \subset \mathbb{D}$, respectively. If $z \in \mathbb{C}$ or $z \in \mathbb{D}$ and $r \geq 0$, then $\mathcal{B}(z, r)$ and $\mathcal{B}_{H}(z, r)$ are the open balls in the Euclidean (respectively, hyperbolic) metric, centered at $z$ and of radius $r ; \overline{\mathcal{B}}(z, r)$ and $\overline{\mathcal{B}}_{H}(z, r)$ are the corresponding closed balls.

The symbol card denotes the number of elements of a finite or a countable set; clos and Int denote, respectively, the closure and interior of a set in a topological space.

If $\Omega \subset \mathbb{R}^{2}$ is an open set and $p \in[1,+\infty]$, then $W^{1, p}(\Omega)$ is the usual Sobolev space of scalar functions $u: \Omega \rightarrow \mathbb{R}$ integrable with exponent $p$ in $\Omega$ and having in $\Omega$ generalized partial derivatives lying in $L^{p}(\Omega)$. The space $W_{\text {loc }}^{1, p}(\Omega)$ is the class of functions belonging to $W^{1, p}(\widetilde{\Omega})$ for any strictly inner open subset $\widetilde{\Omega} \subset \Omega$.

If $X, Y$ are some quantities, then the estimate $X \asymp Y$ means that $C_{1} X \leq Y \leq C_{2} X$ with some absolute constants $C_{1}, C_{2}>0$. In all the other cases of comparability it will be indicated explicitly on what the comparability constants depend.

\section{§1. Statement of the interpolation problem. Simplest properties}

1.1. Periods of a 1-form square-integrable in a regular domain. We fix the class of domains under consideration.

Let $\left\{B_{j}\right\}_{j=1}^{\infty}$ be a countable family of compact subsets of the open unit disk $\mathbb{D} \subset \mathbb{R}^{2} \simeq \mathbb{C}$. We shall assume the following:

(1) the sets $B_{j}$ are pairwise disjoint;

(2) every $B_{j}$ is connected and does not separate the plane; 
(3) every $B_{j}$ is a closure of a domain with smooth boundary (this condition is technical, we shall give it up in Subsection 6.2);

(4) the sets $B_{j}$ accumulate only to the boundary $\partial \mathbb{D}$ of the unit disk $\mathbb{D}$; in other words, if $j=1,2, \ldots$ is fixed, then $\inf _{j^{\prime} \neq j} \operatorname{dist}\left(B_{j^{\prime}}, B_{j}\right)$ is positive (the symbol $\operatorname{dist}\left(E_{1}, E_{2}\right)$ denotes the Euclidean distance between two sets $\left.E_{1}, E_{2} \subset \mathbb{R}^{2}\right)$.

Consider the set $\Omega=\mathbb{D} \backslash\left(\bigcup_{j=1}^{\infty} B_{j}\right)$; this is a countably-connected domain. The sets $B_{j}$, i.e., the bounded connected components of $\Omega^{(c)}$, are called the holes in $\Omega$. Any set $\Omega$ of this sort will be called a regular domain. In the case where all $B_{j}$ are closed disks, we say that $\Omega$ is a regular domain with round holes. Sometimes, we consider domains with finite number of holes. If the holes in such a domain satisfy the above-listed conditions, then we also say that such a domain is regular (investigation of such domains differs from studying of countably-connected domains by an obvious change of notation).

Recall that a (real) differential form of degree 1 (1-form) on some set $\Omega \subset \mathbb{R}^{2}$ is an object $\omega=\omega_{x} d x+\omega_{y} d y$, where $\omega_{x}, \omega_{y}: \Omega \rightarrow \mathbb{R}$ are functions called the components of the form $\omega$. Put $|\omega|:=\sqrt{\omega_{x}^{2}+\omega_{y}^{2}}$. The Hodge star operator $*$ maps a 1 -form $\omega$ to the 1 -form $* \omega=\omega_{x} d y-\omega_{y} d x$; we have $* * \omega=-\omega$.

In what follows we consider forms with Lebesgue measurable components. A smooth 1 -form $\omega$ defined on an open set $\Omega$ said to be closed if $d \omega=0$, where $d$ is the usual differential of the form. If components of $\omega$ are nonsmooth but locally integrable in $\Omega$, then we say that $\omega$ is closed if $d \omega=0$ in the sense of distributions. This means that

$$
\int_{\Omega} \omega \wedge d \eta=0
$$

for any "test" function $\eta \in C_{0}^{\infty}(\Omega)$. A form $\omega$ with components in $L_{\text {loc }}^{1}(\Omega)$ is said to be exact if there exists a function $u \in W_{\text {loc }}^{1,1}(\Omega)$ such that $d u=\omega$ in the sense of distributions. On smooth forms, the classical notions of closeness and exactness coincide with their distributional analogs. Next, a 1-form $\omega$ defined on an open set $\Omega$ is coclosed (or coexact) if the form $* \omega$ is closed (respectively, exact). We do not make use of the codifferential operator $* d *$.

Let $U \subset \mathbb{R}^{2}$ be an open subset, let $E \subset \mathbb{R}^{2}$, and let $\varphi: U \rightarrow E$ be a $C^{1}$-smooth mapping. Denote by $J_{\varphi}(z)$ the Jacobian of the mapping $\varphi$ in $z \in U$, and let $D \varphi(z)$ be its differential with respect to $z ;|D \varphi(z)|$ is the norm of that differential as the norm of a matrix of size $2 \times 2$ with respect to the Euclidean norm in $\mathbb{R}^{2}$.

If a form $\omega$ is defined on $E$, then the form $\varphi^{\sharp} \omega$ on $U$ can be defined by the standard pull-back of $\omega$ obtained by a change of coordinates. If a 1 -form $\widetilde{\omega}=\widetilde{\omega}_{x} d x+\widetilde{\omega}_{y} d y$ is understood as a row-vector with components $\omega_{x}$ and $\omega_{y}$, then $\left(\varphi^{\sharp} \omega\right)(z)=\omega(\varphi(z)) \cdot D \varphi(z)$. If $E$ is open, then the pull-back and the differential commute. In particular, the pull-back of a closed (or exact) form if closed (respectively, exact).

Let

$$
L^{2,1}(\Omega)=\left\{\omega-1 \text {-form in } \Omega,\|\omega\|_{L^{2,1}(\Omega)}^{2}:=\int_{\Omega}|\omega|^{2} d \lambda_{2}<+\infty\right\}
$$

be the set of all differential forms of degree 1 defined in $\Omega$ and square-integrable over the area measure (the symbol $\lambda_{2}$ denotes the two-dimensional Lebesgue measure on the plane). The space $L^{2,1}(\Omega)$ is endowed with the scalar product

$$
\begin{aligned}
\left\langle\omega_{1}, \omega_{2}\right\rangle_{L^{2,1}(\Omega)} & :=\int_{\Omega} \omega_{1} \wedge * \omega_{2}=\int_{\Omega}\left(\omega_{1 x} \omega_{2 x}+\omega_{1 y} \omega_{2 y}\right) d \lambda_{2}, \quad \omega_{1}, \omega_{2} \in L^{2,1}(\Omega), \\
\omega_{1} & =\omega_{1 x} d x+\omega_{1 y} d y, \quad \omega_{2}=\omega_{2 x} d x+\omega_{2 y} d y,
\end{aligned}
$$

turning it into a Hilbert space. 
Let

$$
L_{c}^{2,1}(\Omega)=\left\{\omega \in L^{2,1}(\Omega): d \omega=0\right\}
$$

be the subspace consisting of all closed forms of class $L^{2,1}(\Omega)$; here the equation $d \omega=0$ is understood in the sense of distributions, see (1). The subspace $L_{c}^{2,1}(\Omega)$ is closed in $L^{2,1}(\Omega)$. The scalar product in $L_{c}^{2,1}(\Omega)$ is inherited from $L^{2,1}(\Omega)$.

Let $j=1,2, \ldots$ be an index. Since the sets $B_{j^{\prime}}$ with $j^{\prime} \neq j$ do not accumulate to $B_{j}$, one can find a smooth closed oriented curve $\gamma_{j} \subset \Omega$ winding around $B_{j}$ once in the positive direction and not winding around the other holes $B_{j^{\prime}}$. For forms $\omega \in L_{c}^{2,1}(\Omega)$ smooth in $\Omega$, we define the period functional $\operatorname{Per}_{j}$ by putting

$$
\operatorname{Per}_{j}(\omega):=\int_{\gamma_{j}} \omega
$$

This functional will not change if we replace $\gamma_{j}$ by another curve compactly homological (see [4]) to $\gamma_{j}$ in $\Omega$.

Proposition 1.1 (see also [15]). The functional $\mathrm{Per}_{j}$ has a unique continuous extension from the closed $L^{2}$-forms smooth in $\Omega$ to the entire $L_{c}^{2,1}(\Omega)$.

The density of the smooth closed forms in $L_{c}^{2,1}(\Omega)$ follows, for example, from the Hodge decomposition (see Subsection 2.1). The continuity of the functional $\operatorname{Per}_{j}$ with respect to the $L^{2}$-norm can easily be derived from the fact that the curve $\gamma_{j}$ is contained in $\Omega$ with some neighborhood. The integrals of a closed form over homological loops coincide, while an appropriate average of the family of curves compactly homological to $\gamma_{j}$ in $\Omega$ will be absolutely continuous with respect to $\lambda_{2}$ and will have bounded density with respect to $\lambda_{2}$.

Note that if $\omega \in L_{c}^{2,1}(\Omega)$, then the integral $\int_{\gamma} \omega$ is well defined and coincides with $\operatorname{Per}_{j} \omega$ for 2-quasievery smooth loop $\gamma \subset \Omega$ homological in $\Omega$ to $\gamma_{j}$ (see [15]).

Now we define the period operator Per: $L_{c}^{2,1}(\Omega) \rightarrow \ell^{2}$ by putting

$$
\operatorname{Per} \omega:=\left\{\operatorname{Per}_{j}(\omega)\right\}_{j=1}^{\infty}, \quad \omega \in L_{c}^{2,1}(\Omega),
$$

(in what follows $\ell^{2}$ is the standard space of real square-summable number sequences). In other words, the operator Per maps a closed form to the sequence of its periods around the holes $B_{j}$. When dealing with several regular domains, we shall sometimes need to emphasize the dependence of the operator Per on $\Omega$; then we denote it by $\operatorname{Per}^{(\Omega)}$, and $\operatorname{Per}_{j}^{(\Omega)}$ will stand for its components that are the corresponding period functionals. Mainly, we shall work with one domain and such a notation will not be needed.

As in the case of smooth forms, by extending a primitive over paths, one can show that if $\omega \in L_{c}^{2,1}(\Omega)$ and Per $\omega=0$, then there exists a function $u \in W_{\text {loc }}^{1,2}(\Omega)$ such that $\omega=d u$ in the sense of distributions.

Definition 1.2. Let $\Omega$ be a regular domain.

(1) We say that $\Omega$ possesses the Bessel property if the operator Per: $L_{c}^{2,1}(\Omega) \rightarrow \ell^{2}$ is bounded. The norm of this operator will be called the Bessel constant of the domain $\Omega$ and is denoted by $C_{B}(\Omega)$.

(2) If the quantity $\widetilde{C}_{B}(\Omega):=\sup _{j \in \mathbb{N}}\left\|\operatorname{Per}_{j}\right\|_{\left(L_{c}^{2,1}(\Omega)\right)^{*}}$ is finite, then we say that $\Omega$ possesses the weak Bessel property. The quantity $\widetilde{C}_{B}(\Omega)$ will be called the weak Bessel constant of $\Omega$.

(3) We say that $\Omega$ possesses the interpolation property if for any sequence $a \in \ell^{2}$ there exists a form $\omega \in L_{c}^{2,1}(\Omega)$ such that Per $\omega=a$ and $\|\omega\|_{L_{c}^{2,1}}(\Omega) \leq C \cdot\|a\|_{\ell^{2}}$ with some $C<+\infty$ independent of $a$. The smallest such $C$ will be called the interpolation constant of $\Omega$ and is denoted by $C_{I}(\Omega)$. In other words, the 
interpolation property means that the operator Per: $L_{c}^{2,1}(\Omega) \rightarrow \ell^{2}$ has a bounded right inverse, whereas $C_{I}(\Omega)$ is the smallest norm of a right inverse of Per.

(4) If $\Omega$ has both Bessel and interpolation properties, then we say that $\Omega$ has the complete interpolation property.

Of course, the Bessel property implies the weak Bessel property with $\widetilde{C}_{B}(\Omega) \leq C_{B}(\Omega)$. We shall see below (Theorem 2.16) that the weak Bessel property implies the Bessel property with $C_{B}(\Omega) \leq \sqrt{2} \widetilde{C}_{B}(\Omega)$.

Every functional $\operatorname{Per}_{j}$ is bounded in $L_{c}^{2,1}(\Omega)$, so that Per is a closed operator. So, the interpolation property is equivalent to the surjectivity of the operator Per: $L_{c}^{2,1}(\Omega) \rightarrow \ell^{2}$.

The problem on interpolation by periods is to describe the regular domains $\Omega$ that have the Bessel, interpolation, and complete interpolation properties and also to estimate the constants $C_{B}(\Omega)$ and $C_{I}(\Omega)$ via metric characteristics of the mutual layout of the sets $B_{j}$.

If the reader wants to get immediate constructions of domains with complete interpolation property, then he (or she) can look at Examples 3.3 and 3.3 in Subsection 3.3 . Estimates in those examples are straightforward and use only the definition given above and also the conformal invariance of our problem (Proposition 1.3).

Note that we do not pose the question about interpolation by the normed periods

$$
\operatorname{Per}_{j}(\omega) /\left\|\operatorname{Per}_{j}\right\|_{\left(L_{c}^{2,1}(\Omega)\right)^{*}},
$$

as it is usually done in the classical problems on analytic interpolation. In these problems, normalization of the sequence of values of analytic functions allows one to take the speed of tendency of interpolation nodes to the boundary into account, whereas the reproducing kernels of value functionals can often be found in an explicit form (or, at least, the asymptotics for the norms of these functionals can be calculated). Below we shall see that the norms of the functionals $\mathrm{Per}_{j}$ (or, which is the same, the norms of their reproducing kernels) cannot be found in a completely explicit manner, and also, these norms may turn out to be either arbitrarily small or arbitrarily large - without any relationship to the distance from the holes $B_{j}$ to the unit circle $\partial \mathbb{D}$. One of our goals is to find conditions under which such norms are bounded from above as well as from below.

Now it should be noted that our problem can be stated more generally. First, instead of the disk $\mathbb{D}$ we may take any other domain $V \subset \mathbb{C}$ or even a Riemann surface (while the sets $B_{j}$ would be holes in $V$ ). If $V$ is simply connected, then, by the Riemann uniformization theorem, we may reduce such a problem to the case of a disk with holes (see also Proposition 1.3 on the conformal invariance of our problem); investigation of a uniformizing mapping may, in general, turn out to be difficult (we note that the techniques employed for this are similar to those that we use below). We do not study the problem in such a generality. Nevertheless, in Subsection 3.2 we invoke a conformal mapping of an annulus in $\mathbb{D}$ onto an appropriate cylinder. This allows us to simplify calculations.

Second, we may calculate periods along other curves, not only those winding around the corresponding holes $B_{j}$. Namely, let $\Omega \subset \mathbb{C}$ be an arbitrary domain (one may also consider Riemann surfaces), and let $\gamma_{j}, j=1,2, \ldots$, be some smooth oriented closed curves in $\Omega$. The space $L_{c}^{2,1}(\Omega)$ in this case is defined as above. Every curve $\gamma_{j}$ lies in $\Omega$ together with some neighborhood, and thus the functional $\operatorname{Per}_{j}: L_{c}^{2,1}(\Omega) \rightarrow \mathbb{R}$, $\operatorname{Per}_{j}(\omega)=\int_{\gamma_{j}} \omega, \omega \in L_{c}^{2,1}(\Omega)$, is well defined and continuous. The questions on the Bessel and interpolation properties in this case are stated as above. We shall say that an interpolation problem is posed in $\Omega$ for the periods along the curves $\gamma_{j}$; the constants $C_{B}(\Omega)=C_{B}\left(\Omega ; \gamma_{1}, \gamma_{2}, \ldots\right)$ and $C_{I}(\Omega)=C_{I}\left(\Omega ; \gamma_{1}, \gamma_{2}, \ldots\right)$ are defined as above. This 
problem does not change if we replace every curve $\gamma_{j}$ by another curve equal to $\gamma_{j}$ in the compact homology space $H_{1}^{c}(\Omega)$ (see [4]).

The way described above to choose curves $\gamma_{j}$ corresponding to the holes $B_{j}$ seems to be the most natural from an analytical viewpoint. The curves $\gamma_{j}$ are linearly independent and complete in the space of compact homologies (the last property means that if $\int_{\gamma_{j}} \omega=0$ for all $j=1,2, \ldots$, then the form $\omega \in L_{c}^{2,1}(\Omega)$ is exact). At the same time, if we start with the intrinsic geometry of the domain $\Omega$ (as a Riemann surface), then we cannot make any canonical choice of a complete linearly independent system of compact homologies in $\Omega$. In particular, if we choose curves $\gamma_{j}$ corresponding to the holes $B_{j}$ in $\Omega$ and make an inversion $\varphi$ with the center in one of the holes $B_{j}$, then the domain will turn inside out and the curves $\varphi\left(\gamma_{j}\right)$ will not correspond to the holes in $\varphi(\Omega)$. We shall meet such a situation in examples in Subsection 3.3 (see Example 3.3 and Figure 8 and also Example 4). In most cases, we shall still deal with the problem on interpolation by periods over curves corresponding to the holes $B_{j}$. We shall assume this tacitly whenever the choice of the curves $\gamma_{j}$ is not specified.

1.2. (Quasi)conformal (quasi)invariance of the problem. We recall the definition of a quasiconformal mapping; we restrict ourselves to the smooth case. Let $\Omega, \widetilde{\Omega} \subset \mathbb{R}^{2}$ be domains, let $\varphi: \Omega \rightarrow \widetilde{\Omega}$ be a $C^{\infty}$-smooth diffeomorphism, and let $K \geq 1$ be a number. The mapping $\varphi$ is said to be $K$-quasiconformal if for any $z \in \Omega$ we have

$$
|D \varphi(z)|^{2} \leq K \cdot J_{\varphi}(z) \text {. }
$$

The smallest constant $K$ for which (3) is true is called the distortion coefficient of $\varphi$ and is denoted by $K(\varphi)$. The definition of quasiconformity, in particular, implies that $J_{\varphi}(z) \geq 0$ for all $z \in \Omega$, that is, a quasiconformal mapping preserves orientation. Also, a 1-quasiconformal mapping is conformal. See, e.g., 1] for more information on quasiconformal mappings.

A (quasi)conformal change of variables easily leads to the following statement.

Proposition 1.3. Suppose that an interpolation problem is stated in a domain $\Omega$ for periods along curves $\gamma_{j}, j=1,2, \ldots$ (see the end of Subsection 1.1) and that $\widetilde{\Omega}$ is a domain in $\mathbb{R}^{2}$. Suppose that $\varphi: \Omega \rightarrow \widetilde{\Omega}$ is an orientation preserving diffeomorphism. We pose the interpolation problem in $\widetilde{\Omega}$ for periods along the curves $\varphi\left(\gamma_{j}\right), j=1,2, \ldots$

(1) If $\varphi$ is conformal, then $C_{B}(\widetilde{\Omega})=C_{B}(\Omega)$ and $C_{I}(\widetilde{\Omega})=C_{I}(\Omega)$.

(2) If $\varphi$ is quasiconformal and $K=K(\varphi)$ is its distortion coefficient, then

$$
\begin{aligned}
& \sqrt{K^{-1}} \cdot C_{B}(\Omega) \leq C_{B}(\widetilde{\Omega}) \leq \sqrt{K} \cdot C_{B}(\Omega), \\
& \sqrt{K^{-1}} \cdot C_{I}(\Omega) \leq C_{I}(\widetilde{\Omega}) \leq \sqrt{K} \cdot C_{I}(\Omega), \\
& \sqrt{K^{-1}} \cdot \widetilde{C}_{B}(\Omega) \leq \widetilde{C}_{B}(\widetilde{\Omega}) \leq \sqrt{K} \cdot \widetilde{C}_{B}(\Omega) .
\end{aligned}
$$

In other words, the problem on interpolation by periods is conformally invariant and quasiconformally quasiinvariant.

1.3. Forms that minimize $\|\omega\|_{L_{c}^{2,1}(\Omega)}$ under prescribed periods. It is natural to reformulate the questions on the Bessel and interpolation properties of a regular domain $\Omega$ as follows: what is the minimal $L^{2}$-norm of a closed 1 -form with given periods? can this norm be estimated from below (respectively, from above) in terms of the sum of squares of the periods? We shall need some simple properties of such norm minimizers.

Proposition 1.4. Let $\Omega$ be a regular domain, and let $a=\left\{a_{j}\right\}_{j=1}^{\infty}, a_{j} \in \mathbb{R}, j=1,2, \ldots$, be a sequence of scalars. Suppose that the set $Y=\left\{\omega \in L_{c}^{2,1}(\Omega): \operatorname{Per} \omega=a\right\}$ is not empty. Then there exists a unique element $\omega_{0}(a) \in Y$ with minimal $L_{c}^{2,1}(\Omega)$-norm. 
Proof. The existence of $\omega_{0}(a)$ follows from the closedness of the affine subspace $Y \subset$ $L_{c}^{2,1}(\Omega)$. Uniqueness follows from the fact that a sphere in a Hilbert space contains no segments.

Remark. The Bessel property of a domain $\Omega$ is equivalent to the estimate $\left\|\omega_{0}(a)\right\|_{L_{c}^{2,1}(\Omega)} \geq$ $C_{B}^{-1}(\Omega) \cdot\|a\|_{\ell^{2}}$ for any $a \in \operatorname{Per}\left(L_{c}^{2,1}(\Omega)\right)$. The interpolation property of a domain $\Omega$ is fulfilled if and only if, for any $a \in \ell^{2}$, the form $\omega_{0}(a)$ is well defined and $\left\|\omega_{0}(a)\right\|_{L_{c}^{2,1}(\Omega)} \leq$ $C_{I}(\Omega) \cdot\|a\|_{\ell^{2}}$.

Proposition 1.5. For any real sequence $a \in \operatorname{Per}\left(L_{c}^{2,1}(\Omega)\right)$, the form $\omega_{0}(a)$ defined as in Proposition 1.4 is co-exact. Moreover, whenever $\omega \in L_{c}^{2,1}(\Omega)$ and $\operatorname{Per} \omega=0$, we have $\left\langle\omega_{0}(a), \omega\right\rangle_{L_{c}^{2,1}(\Omega)}=0$.

The proof is given in the Appendix.

We recall the definition of the class of harmonic forms.

Definition 1.6. A form $\omega \in L^{2,1}(\Omega)$ is said to be harmonic if

$$
\left\{\begin{array}{c}
d \omega=0 \\
d * \omega=0
\end{array}\right.
$$

on $\Omega$ (the differential relations are understood in the sense of distributions). The space of all 1-forms from $L^{2,1}(\Omega)$ harmonic in $\Omega$ is denoted by $\operatorname{Harm}^{2,1}(\Omega)$.

The forms $\omega_{0}(a)$ associated with real sequences $a$ as in Proposition 1.4 are closed and co-exact, so that they are harmonic. Since the components of harmonic forms are harmonic functions, any form harmonic in $\Omega$ is smooth in $\Omega$.

1.4. Interpolation by periods in $L_{c}^{2,1}(\Omega)$ and in Bergman space. Let $\Omega$ be a regular domain, and let

$$
\mathscr{A}(\Omega)=\left\{f: \Omega \rightarrow \mathbb{C} \mid f \text { is analytic in } \Omega,\|f\|_{\mathscr{A}(\Omega)}^{2}=\int_{\Omega}|f|^{2} d \lambda_{2}<+\infty\right\}
$$

be the (unweighted) Bergman space in $\Omega$. With any function $f \in \mathscr{A}(\Omega)$ we associate the sequence $\left\{\int_{\gamma_{j}} f(\zeta) d \zeta\right\}_{j=1}^{\infty}$ of its periods along the curves $\gamma_{j}$ chosen as above. The problem on interpolation of functions in the Bergman space in $\Omega$ by their periods is stated by analogy with the problem on forms (but the period operator is viewed as an operator from $\mathscr{A}(\Omega)$ to the space $\ell_{\mathbb{C}}^{2}$ of complex sequences). Let $C_{B, \mathscr{A}}(\Omega)$ and $C_{I, \mathscr{A}}(\Omega)$ be the Bessel and interpolation constants for the period operator in the space $\mathscr{A}(\Omega)$ defined in the same way as in the problem on 1-forms.

Notice that a form $\omega=\omega_{1} d x+\omega_{2} d y$ defined in $\Omega$ is harmonic if and only if the function $f=\omega_{1}-i \omega_{2}$ is analytic in $\Omega$. From this observation and results of Subsection 1.3 it is not hard to derive the following statement.

Proposition 1.7. Let $\Omega$ be a regular domain.

(1) A domain $\Omega$ has the Bessel property for $L_{c}^{2,1}(\Omega)$ if and only if it has the Bessel property for $\mathscr{A}(\Omega)$. Also, $C_{B}(\Omega)=C_{B, \mathscr{A}}(\Omega)$.

(2) A domain $\Omega$ has the interpolation property for $L_{c}^{2,1}(\Omega)$ if and only if it has the interpolation property for $\mathscr{A}(\Omega)$. Also, $C_{I}(\Omega)=C_{I, \mathscr{A}}(\Omega)$.

Thus, our problem is equivalent to the problem in the Bergman space. The multiplier techniques used for the investigation of interpolation problems in spaces of analytic functions seem to fail in the case of periods, as well as the Blaschke product techniques. Thus, in what follows, we shall not make use of analytic functions. 


\section{§2. Preliminary Results}

In Subsection 2.1 we consider the Hodge decomposition of the space $L^{2,1}(\Omega)$. This will allow us to specify the subspace of $L_{c}^{2,1}(\Omega)$ that contains the reproducing kernels of the functionals $\operatorname{Per}_{j}, j=1,2, \ldots$ We shall find these kernels by solving the Dirichlet problem for the Laplace operator in Subsection 2.2. In Subsection 2.3 we apply the Riesz conditions to the system of these reproducing kernels: the interpolation problem will be reduced to estimates for functions in $\stackrel{\circ}{W}^{1,2}(\mathbb{D})$ that are constant on the sets $B_{j}$, $j=1,2, \ldots$ In Subsection 2.5 we use these estimates to deduce some capacity conditions necessary for complete interpolation. In Subsection 2.7 we give metric estimates that link the Euclidean and hyperbolic metrics to condenser capacities. Also in Subsection 2.7 we introduce conditions of weak (or strong) separation of holes; these conditions turn out to be necessary (or sufficient) for the Bessel property of $\Omega$.

2.1. Hodge decomposition. For compact manifolds without boundary, a Hodge decomposition with three components is usually written. We shall need four components of this decomposition. In this subsection all the orthogonal sums and orthogonal complements are understood in the sense of the scalar product in $L^{2,1}(\Omega)$.

Let $\Omega$ be a regular domain (or even a Riemann manifold). The set $\operatorname{Harm}^{2,1}(\Omega)$ of harmonic forms of class $L^{2,1}(\Omega)$ was introduced at the end of Subsection 1.3. It is well known that

$$
\begin{aligned}
L^{2,1}(\Omega)= & \operatorname{clos}_{L^{2,1}(\Omega)}\left\{d u: u \in C_{0}^{\infty}(\Omega)\right\} \\
& \oplus \operatorname{clos}_{L^{2,1}(\Omega)}\left\{* d u: u \in C_{0}^{\infty}(\Omega)\right\} \oplus \operatorname{Harm}^{2,1}(\Omega) \\
= & \mathcal{F}_{1}(\Omega) \oplus \mathcal{F}_{2}(\Omega) \oplus \operatorname{Harm}^{2,1}(\Omega) .
\end{aligned}
$$

Indeed, the Stokes theorem immediately gives the orthogonality of the first two terms of this decomposition. Next, if a form $\omega$ is orthogonal to any form in the first two components, then $\omega$ is closed and co-closed in the sense of distributions and, thus, is harmonic (and smooth) in $\Omega$.

Proposition 2.1. If $\omega \in L^{2,1}(\Omega)$ and $d \omega=0$, then $\omega \perp \mathcal{F}_{2}(\Omega)$ in $L^{2,1}(\Omega)$. In other words,

$$
L_{c}^{2,1}(\Omega)=\mathcal{F}_{1}(\Omega) \oplus \operatorname{Harm}^{2,1}(\Omega) .
$$

Proof. The equation $\int_{\Omega}\langle\omega, * d u\rangle d \lambda_{2}=0$ for all $u \in C_{0}^{\infty}(\Omega)$ is exactly the distributional form of the identity $d \omega=0$.

Now we refine the decomposition (44). We put

$$
\mathcal{F}_{3}(\Omega)=\left\{\omega \in \operatorname{Harm}^{2,1}(\Omega): \omega \text { is exact in } \Omega\right\}
$$

and $\mathcal{F}_{4}(\Omega)=\operatorname{Harm}^{2,1}(\Omega) \ominus \mathcal{F}_{3}(\Omega)$. Then

$$
\operatorname{Harm}^{2,1}(\Omega)=\mathcal{F}_{3}(\Omega) \oplus \mathcal{F}_{4}(\Omega)
$$

and finally

$$
L^{2,1}(\Omega)=\mathcal{F}_{1}(\Omega) \oplus \mathcal{F}_{2} \oplus \mathcal{F}_{3}(\Omega) \oplus \mathcal{F}_{4}(\Omega) .
$$

Proposition 2.2. Denote by $\mathcal{F}_{5}(\Omega)$ the set of all exact forms of class $L^{2,1}(\Omega)$. Then

$$
\mathcal{F}_{4}(\Omega)=\operatorname{Harm}^{2,1}(\Omega) \cap\left(L^{2,1}(\Omega) \ominus \mathcal{F}_{5}(\Omega)\right) .
$$

In other words, the forms in $\mathcal{F}_{4}(\Omega)$ are orthogonal not only to the exact harmonic forms but also to all square-integrable exact forms. 
The proof is given in the Appendix.

In the case where $\Omega$ is a compact manifold with sufficiently smooth boundary, the forms $\omega \in \mathcal{F}_{4}(\Omega)$ can be described in terms of their boundary data (namely, the normal component of $\omega$ on $\partial \Omega$ must vanish). Moreover, in this case the space $\mathcal{F}_{4}(\Omega)$ is finitedimensional (see, e.g., [17]). If, to the contrary, the boundary $\partial \Omega$ of a domain $\Omega \subset \mathbb{R}^{2}$ is nonsmooth, then the vanishing of normal components of forms makes no sense; moreover, the space $\mathcal{F}_{4}(\Omega)$ may turn out to be infinite-dimensional. This will occur for infinitely connected regular domains.

Proposition 2.1 and the explicit form of the spaces $\mathcal{F}_{1}(\Omega)$ and $\mathcal{F}_{3}(\Omega)$ easily imply the next claim.

Proposition 2.3. Let $\omega \in L_{c}^{2,1}(\Omega)$ be a form, and $\omega_{4}$ the projection of $\omega$ to $\mathcal{F}_{4}(\Omega)$ in $L^{2,1}(\Omega)$. Then $\operatorname{Per} \omega=\operatorname{Per} \omega_{4}$. In particular, the operator Per vanishes on the space $\mathcal{F}_{1}(\Omega) \oplus \mathcal{F}_{3}(\Omega)$.

Remark. If $\omega \in \mathcal{F}_{4}(\Omega), \omega \neq 0$, then $\operatorname{Per}_{j}(\omega) \neq 0$ for some $j=1,2, \ldots$ Indeed, otherwise $\omega$ would be exact, whereas all the exact harmonic forms fall in $\mathcal{F}_{3}(\Omega)$ but not in $\mathcal{F}_{4}(\Omega)$.

2.2. Period reproducing kernels. Let $\Omega$ be a regular domain, $j=1,2, \ldots$ We are going to find forms $\kappa_{j} \in L_{c}^{2,1}(\Omega)$ such that

$$
\left\langle\kappa_{j}, \omega\right\rangle_{L_{c}^{2,1}}(\Omega)=\operatorname{Per}_{j}(\omega)
$$

for any $\omega \in L_{c}^{2,1}(\Omega)$. Such a form $\kappa_{j}$ is called the reproducing kernel of the functional Per $_{j}$ (in the space $L_{c}^{2,1}(\Omega)$ ). In the case of a domain (or even a Riemann surface) with smooth boundary, these kernels were known before (see, e.g., [12]). Proposition 2.3 implies that $\kappa_{j} \in \mathcal{F}_{4}(\Omega)$ (because the functional $\operatorname{Per}_{j}$ vanishes on $\mathcal{F}_{1}(\Omega)$ and on $\mathcal{F}_{3}(\Omega)$ ).

In what follows, $\stackrel{\circ}{W}^{1,2}(\mathbb{D})$ is the Sobolev space of functions $u: \mathbb{D} \rightarrow \mathbb{R}$ that have squareintegrable partial distributional derivatives in $\mathbb{D}$ of order 1 and such that $u=0$ on $\partial \mathbb{D}$ in the sense of the boundary values operator. The space $\mathscr{W}^{1,2}(\mathbb{D})$ is endowed with the Dirichlet norm:

$$
\|u\|_{\mathscr{W}^{1,2}(\mathbb{D})}:=\left(\int_{\mathbb{D}}|\nabla u|^{2} d \lambda_{2}\right)^{1 / 2}, \quad u \in \stackrel{\circ}{W}^{1,2}(\mathbb{D}) .
$$

Any function in $\stackrel{\circ}{W}^{1,2}(\mathbb{D})$ is assumed to be extended by zero to $\mathbb{D}^{(c)}$ up to a Sobolev class function on the entire plane.

Theorem 2.4. Let $\Omega$ be a regular domain and $j=1,2, \ldots$ an index.

(1) There exists a function $\mathfrak{v}_{j}=\mathfrak{v}_{j}(\Omega) \in W^{1,2}(\mathbb{D})$ such that

$$
\begin{cases}\Delta \mathfrak{v}_{j}=0 & \text { in } \Omega ; \\ \mathfrak{v}_{j}=1 & \text { almost everywhere on } B_{j} ; \\ \mathfrak{v}_{j}=0 & \text { almost everywhere on } B_{j^{\prime}}, j^{\prime} \neq j ; \\ \mathfrak{v}_{j}=0 & \text { on } \partial \mathbb{D} \text { in the sense of the boundary values operator. }\end{cases}
$$

The function $\mathfrak{v}_{j}$ minimizes the Dirichlet integral $\int_{\mathbb{D}}|\nabla v|^{2} d \lambda_{2}$ over all functions $v \in \stackrel{\circ}{W}^{1,2}(\mathbb{D})$ with the same values on $\Omega^{(c)}$.

(2) If $v \in W^{1,2}(\mathbb{C}), v=0$ almost everywhere in $\Omega^{(c)}$, and $\Delta v=0$ in $\Omega$, then $v=0$.

(3) The function $\mathfrak{v}_{j}$ satisfying (7) is unique.

(4) The reproducing kernel of the functional $\operatorname{Per}_{j}$ on $L_{c}^{2,1}(\Omega)$ is the form $\kappa_{j}=-\left(* d \mathfrak{v}_{j}\right)$.

The proof is given in the Appendix.

Note that if the holes $B_{j}$ are, for example, slits, then we cannot state the Dirichlet problem by equating functions to 0 or 1 almost everywhere on the holes. In this case 
the boundary problem should be stated in the class of precised functions. Then equality to 0 or 1 will be up to zero capacity, that is, quasieverywhere. We then need to require the positivity of the capacities of all the holes $B_{j^{\prime}}$. This will be the case if any $B_{j^{\prime}}$ is connected and consists of more than one point.

Our problem can also be stated in terms of vector fields (1-forms are naturally associated with vector fields). Then the periods of forms correspond to the circulations of fields over given curves. In the language of vector fields, the reproducing kernel $-\left(* d \mathfrak{v}_{j}\right)$ corresponds to the field $\left(\nabla \mathfrak{v}_{j}\right)^{\perp}$, which is the gradient of the function $\mathfrak{v}_{j}$ turned over $\pi / 2$ counterclockwise.

Remark. By the remark after Proposition 2.3 the system $\left\{\kappa_{j}\right\}_{j=1}^{\infty}$ will be complete in the space $\mathcal{F}_{4}(\Omega)$.

In the proof of Theorem 2.4 we use the following inequality (we shall need it also in some other situations).

Theorem 2.5. There exists a constant $\mathfrak{c}>0$ such that

$$
\int_{\mathbb{D}} \frac{u^{2}(z) d \lambda_{2}(z)}{(1-|z|)^{2}} \leq \mathfrak{c} \cdot \int_{\mathbb{D}}|\nabla u|^{2} d \lambda_{2}
$$

for any function $u \in \stackrel{\circ}{W}^{1,2}(\mathbb{D})$. In other words, the embedding $\stackrel{\circ}{W}^{1,2}(\mathbb{D}) \hookrightarrow L^{2}\left(\mu_{0}\right)$, where $\mu_{0}=\frac{\lambda_{2}}{(1-|z|)^{2}}$, is continuous.

Indeed, it suffices to estimate $\int_{|z| \geq 1 / 2} \frac{u^{2}(z) d \lambda_{2}(z)}{(1-|z|)^{2}}$. But this is easy to do by applying the well-known Hardy inequality

$$
\int_{0}^{\infty}\left(\frac{1}{x} \int_{0}^{x} f(y) d y\right)^{2} d x \leq 4 \int_{0}^{\infty} f^{2}(y) d y, \quad f \in L^{2}[0,+\infty)
$$

(see, e.g., 6] ) to the function $f(r)=\frac{\partial}{\partial r} u\left((1-r) e^{i \theta}\right)$ on the segment $\left[0, \frac{1}{2}\right]$ and integrating the resulting estimate over $\theta \in[0,2 \pi)$.

Note, by the way, that the full mass of the measure $\mu_{0}$ is infinite, in particular, the estimate in Theorem 2.5 fails for $u \equiv 1$ (even if we replace the right-hand side of this inequality by the whole Sobolev norm). In other words, the zero boundary data of $u$ on $\partial \mathbb{D}$ is essential for the validity of this estimate.

The following surprising observation will easily lead us to the result of Theorem 2.16 (see below).

Proposition 2.6. Suppose that $\Omega$ is a regular domain and $j, j^{\prime}=1,2, \ldots, j \neq j^{\prime}$. Then $\left\langle\kappa_{j}, \kappa_{j^{\prime}}\right\rangle_{L_{c}^{2,1}(\Omega)}<0$ where $\kappa_{j}, \kappa_{j^{\prime}}$ are the period reproducing kernels found in Theorem 2.4 .

The proof is given in the Appendix.

2.3. Riesz property. So, the period reproducing kernels $\kappa_{j} \in \mathcal{F}_{4}(\Omega), j=1,2, \ldots$, are found. We are going to apply the theory of Riesz bases to these kernels. We start with the following statement.

Proposition 2.7. The system $\left\{\kappa_{j}\right\}_{j=1}^{\infty}$ has a biorthogonally adjoint system in $\mathcal{F}_{4}(\Omega)$, which is a sequence of forms $\mathfrak{w}_{j} \in \mathcal{F}_{4}(\Omega), j=1,2, \ldots$, such that $\left\langle\mathfrak{w}_{j}, \kappa_{j}\right\rangle_{L_{c}^{2,1}}(\Omega)=1$ and $\left\langle\mathfrak{w}_{j}, \kappa_{j^{\prime}}\right\rangle_{L_{c}^{2,1}}(\Omega)=0$ for $j \neq j^{\prime}$.

Proof. Pick $z_{j}=x_{j}+i y_{j} \in \operatorname{Int} B_{j}$. Then the form

$$
\omega_{j}(x+i y):=\frac{\left(x-x_{j}\right) d y-\left(y-y_{j}\right) d x}{2 \pi\left(\left(x-x_{j}\right)^{2}+\left(y-y_{j}\right)^{2}\right)}, \quad x+i y \in \Omega,
$$

belongs to $L_{c}^{2,1}(\Omega)$, and $\operatorname{Per}_{j} \omega_{j}=1, \operatorname{Per}_{j} \omega_{j^{\prime}}=0$ for $j^{\prime} \neq j$. Now for $\mathfrak{w}_{j}$ we may take the projection of $\omega_{j}$ to $\mathcal{F}_{4}(\Omega)$. 
In the notation of [2], the Bessel property of $\Omega$ means the Bessel property of the system $\left\{\mathfrak{w}_{j}\right\}_{j=1}^{\infty} \subset \mathcal{F}_{4}(\Omega)$, whereas the interpolation property of $\Omega$ means the Hilbert property of that system. Applying the results of [2], it is not hard to prove the following claim.

Proposition 2.8. Let $\Omega$ be a regular domain, and let $\mathfrak{v}_{j}, j=1,2, \ldots$, be the functions obtained by solving the Dirichlet problem (77).

(1) The Bessel property of $\Omega$ is equivalent to the following condition: for any sequence $\left\{a_{j}\right\}_{j=1}^{\infty} \in \ell^{2}$ the series $\sum_{j=1}^{\infty} a_{j} \mathfrak{v}_{j}$ converges strongly in $\stackrel{\circ}{W}^{1,2}(\mathbb{D})$ and

$$
\left\|\sum_{j=1}^{\infty} a_{j} \mathfrak{v}_{j}\right\|_{\stackrel{\circ}{1,2}(\mathbb{D})^{2}}^{2} \leq C_{B}^{2}(\Omega) \cdot \sum_{j=1}^{\infty} a_{j}^{2} .
$$

(2) The interpolation property of $\Omega$ is equivalent to the estimate

$$
\left\|\sum_{j=1}^{\infty} a_{j} \mathfrak{v}_{j}\right\|_{\stackrel{W}{ }^{1,2}(\mathbb{D})}^{2} \geq C_{I}^{-2}(\Omega) \cdot \sum_{j=1}^{\infty} a_{j}^{2}
$$

for any finitely supported sequence $\left\{a_{j}\right\}_{j=1}^{\infty}$. In the case of the Bessel property, the series $\sum_{j=1}^{\infty} a_{j} \mathfrak{v}_{j}$ converges strongly in $\stackrel{\circ}{W}^{1,2}(\mathbb{D})$ for any $\left\{a_{j}\right\}_{j=1}^{\infty} \in \ell^{2}$, and estimate (8) stays true for all such $\left\{a_{j}\right\}_{j=1}^{\infty}$.

Thus, the complete interpolation property of $\Omega$ is equivalent to the following: for any $a=\left\{a_{j}\right\}_{j=1}^{\infty} \in \ell^{2}$, the series $u=\sum_{j=1}^{\infty} a_{j} \mathfrak{v}_{j}$ converges strongly in $\stackrel{\circ}{W}^{1,2}(\mathbb{D})$ and its sum $u$ admits the estimate

$$
C_{I}^{-1}(\Omega) \cdot\|a\|_{\ell^{2}} \leq\|u\|_{\dot{W}^{1,2}(\mathbb{D})} \leq C_{B}(\Omega) \cdot\|a\|_{\ell^{2}} .
$$

Remark. The system $\left\{\kappa_{j}\right\}_{j=1}^{\infty}$ belongs to $\mathcal{F}_{4}(\Omega)$ and, in accordance with the remark at the end of Subsection 2.1, is complete in $\mathcal{F}_{4}(\Omega)$. Thus, estimate (9) means that in the case of complete interpolation the system $\left\{\kappa_{j}\right\}_{j=1}^{\infty}$ must be a Riesz basis (see [2, 10]) in $\mathcal{F}_{4}(\Omega)$.

By Proposition 2.2, the term $\mathcal{F}_{4}(\Omega)$ in the Hodge decomposition (5) can be identified with the space $H_{L^{2}}^{1}(\Omega)$ of Hilbert cohomologies (or $L^{2}$-cohomologies) in $\Omega$. We define this space as follows:

$$
H_{L^{2}}^{1}(\Omega):=\left\{\omega \in L^{2,1}(\Omega): d \omega=0\right\} /\left\{\omega \in L^{2,1}(\Omega): \omega=d u \text { for some } u \in W_{\text {loc }}^{1,2}(\Omega)\right\} .
$$

We also define the space $H_{1, L^{2}}(\Omega)$ of Hilbert homologies as the dual to $H_{L^{2}}^{1}(\Omega)$. Although $H_{1, L^{2}}(\Omega)$ is canonically isomorphic to $H_{L^{2}}^{1}(\Omega)$, from the viewpoint of homology theory these two spaces should be treated differently.

For $j=1,2, \ldots$, the curve $\gamma_{j}$ linked to the hole $B_{j}$ in the domain $\Omega$ delivers a continuous functional on $H_{L^{2}}^{1}(\Omega)$ and thus can be regarded as an element of $H_{1, L^{2}}(\Omega)$. Thus, $\Omega$ has complete interpolation property if and only if the curves $\gamma_{j}, j=1,2, \ldots$, form a Riesz basis in the space $H_{1, L^{2}}(\Omega)$.

2.4. Admissible functions. Finite linear combinations of kernels $\kappa_{j}$ are locally constant on $\Omega^{(c)}$. We introduce the following function class.

Definition 2.9. We say that a function $u \in \stackrel{\circ}{W}^{1,2}(\mathbb{D})$ is admissible for $\Omega$ if, for any $j=1,2, \ldots$, the function $u$ is constant on $B_{j}$ almost everywhere. The class of all functions admissible for $\Omega$ is denoted by $\mathcal{A} d m(\Omega)$.

The set $\mathcal{A} d m(\Omega)$ endowed with the norm $\|u\|_{W^{1,2}(\mathbb{D})}$ is a Hilbert space, and the functionals $\left.u \mapsto u\right|_{B_{j}}, j=1,2, \ldots$, are continuous on $\mathcal{A d m}(\Omega)$. 
Let $\left\{a_{j}\right\}_{j=1}^{\infty}$ be some sequence of real scalars. Among all $u \in \mathcal{A} d m(\Omega)$ with prescribed values $\left.u\right|_{B_{j}} \equiv a_{j}$, the minimizer of the norm $\|u\|_{W^{1,2}(\mathbb{D})}$ is a function harmonic in $\Omega$ and equal to $a_{j}$ on $B_{j}$. For finitely supported sequences $\left\{a_{j}\right\}_{j=1}^{\infty}$ this function is precisely $\sum_{j=1}^{\infty} a_{j} \mathfrak{v}_{j}$. The following proposition will be our main tool to study interpolation.

Proposition 2.10. Let $\Omega$ be a regular domain.

(1) A domain $\Omega$ has the Bessel property if and only if for any $a=\left\{a_{j}\right\}_{j=1}^{\infty} \in \ell^{2}$ there exists a function $u \in \mathcal{A} d m(\Omega)$ such that $\left.u\right|_{B_{j}}=a_{j}$ and $\|u\|_{W^{1,2}(\mathbb{D})}^{2} \leq C_{B}^{2}(\Omega)$. $\|a\|_{\ell^{2}}^{2}$. It suffices to check this condition only for finitely supported sequences $\left\{a_{j}\right\}_{j=1}^{\infty}$.

(2) A domain $\Omega$ possesses the interpolation property if and only if for any function $u \in \mathcal{A d m}(\Omega)$ we have

$$
\|u\|_{W^{1,2}(\mathbb{D})}^{2} \geq C_{I}^{-2}(\Omega) \cdot \sum_{j=1}^{\infty}\left(\left.u\right|_{B_{j}}\right)^{2} .
$$

Remark. An admissible function $u$ can be understood as a 0 -form whose integrals over any two 0 -simplexes in $\Omega^{(c)}$ homological in $\Omega^{(c)}$ coincide. This links the estimates in Proposition 2.10 to the Alexander-Pontryagin duality known from homology theory.

Proof of Proposition 2.10. The only nontrivial assertion 1 here is that estimate (8) for finite linear combinations of reproducing kernels implies inequality (10) for an arbitrary function $u \in \mathcal{A} d m(\Omega)$. (All the other statements are easily obtained by using the fact that harmonic functions minimize the Dirichlet integral if boundary values are given, and also by the uniqueness of a solution of the Dirichlet problem with zero boundary data, see Theorem 2.4.)

We may assume that the function $u$ for which we want to prove inequality (10) is harmonic in $\Omega$. Indeed, let $u \in \mathcal{A} d m(\Omega)$. The usual variational argument shows that, first, there exists a function $u_{h} \in \stackrel{\circ}{W}^{1,2}(\mathbb{D})$ for which $u_{h}=u$ almost everywhere in $\mathbb{D} \backslash \Omega$ and $\Delta u_{h}=0$ in $\Omega$, and, second, $\left\|u_{h}\right\|_{W^{1,2}(\mathbb{D})} \leq\|u\|_{\mathscr{W}^{1,2}(\mathbb{D})}$. But then estimate (10) for $u$ will follow from this estimate for $u_{h}$.

So, assume that $\Delta u=0$ in $\Omega$. The function $u$ is continuous on any compact subset in $\mathbb{D}$ and its partial derivatives of all orders are continuous in $\Omega$ up to all $\partial B_{j}, j=1,2, \ldots$. This follows from the smoothness of the boundaries $\partial B_{j}$ (see [7]). Define $u$ to be zero outside of $\mathbb{D}$ and assume that $\nabla u=0$ everywhere in $\mathbb{C} \backslash \Omega$. This will allow us to avoid problems with measurability in the construction below.

On $\mathbb{C}$, consider the inner metric generated by the metric $|\nabla u||d z|$ (cf. the proof of Theorem 4.2 below). Namely, for any Lipschitz curve $\Gamma:[0, T] \rightarrow \mathbb{C}(T \in \mathbb{R})$, the integral

$$
L(\Gamma):=\int_{\Gamma}|\nabla u| d \mathcal{H}^{1}=\int_{0}^{T}|\nabla u(\Gamma(t))| \cdot\left|\Gamma^{\prime}(t)\right| d t \in[0,+\infty]
$$

is well defined (the expression under the last integral is measurable because the function $|\nabla u(\Gamma)|$ vanishes outside of the open set $\Gamma^{-1}(\Omega) \subset[0, T]$ and is continuous on this set). For $z_{1}, z_{2} \in \mathbb{C}$, put $\rho\left(z_{1}, z_{2}\right):=\inf L(\Gamma)$, where the infimum is taken over all Lipschitz curves in $\mathbb{C}$ joining $z_{1}$ and $z_{2}$. Then $\rho$ is a degenerate metric in $\mathbb{C}$ in which the holes $B_{j}$ collapse to points (any two points $z_{1}, z_{2} \in \Omega^{(c)}$ lying in the same hole $B_{j}$ can be joined by a Lipschitz curve $\Gamma \subset B_{j}$, so that we have $L(\Gamma)=0$ for such a curve); the exterior $\mathbb{D}^{(c)}$ of the unit disk also collapses to a point. The triangle inequality for $\rho$ is nevertheless fulfilled.

\footnotetext{
${ }^{1}$ The reader may be amazed by the length of the following proof of this fact which, at a glance, seems to be obvious. Nevertheless, the author could not find a simpler and more abstract argument.
} 
Pick some $\nu \in \mathbb{N}$. Put

$$
u_{\nu}(z):=\operatorname{dist}_{\rho}\left(z, \mathbb{D}^{(c)} \cup \bigcup_{j=\nu}^{\infty} B_{j}\right), \quad z \in \mathbb{C} .
$$

In other words, $u_{\nu}(z)=\inf L(\Gamma)$, where the infimum is taken over all Lipschitz curves joining $z$ with a point in $\mathbb{D}^{(c)}$ or with a point in one of the sets $B_{j}, j=\nu, \nu+1, \ldots$ If $z_{1}$, $z_{2} \in \Omega$, then we may join these points by a segment $\left[z_{1}, z_{2}\right]$ of a line and conclude that

$$
\left|u_{\nu}\left(z_{1}\right)-u_{\nu}\left(z_{2}\right)\right| \leq \rho\left(z_{1}, z_{2}\right) \leq L\left(\left[z_{1}, z_{2}\right]\right) \leq\left|z_{1}-z_{2}\right| \cdot \sup _{\left[z_{1}, z_{2}\right]}|\nabla u(z)| .
$$

This inequality and the local boundedness of $\nabla u$ in $\mathbb{D}$ imply that the function $u_{\nu}$ is locally Lipschitz in $\mathbb{D}$. If $z_{1}$ belongs to $\Omega$ or to one of the sets $\operatorname{Int} B_{j}, j=1,2, \ldots$, then the gradient $\nabla u$ is continuous in $z_{1}$; this and (11) imply that

$$
\limsup _{z_{2} \rightarrow z_{1}} \frac{\left|u_{\nu}\left(z_{1}\right)-u_{\nu}\left(z_{2}\right)\right|}{\left|z_{1}-z_{2}\right|} \leq\left|\nabla u\left(z_{1}\right)\right| \text {. }
$$

Thus,

$$
\left|\nabla u_{\nu}(z)\right| \leq|\nabla u(z)|
$$

for almost all $z \in \mathbb{D}$. In particular, $u_{\nu}$ is constant on any $B_{j}, j=1,2, \ldots$ Also, (12) implies that $u_{\nu} \in W^{1,2}(\mathbb{D})$ (because $|\nabla u| \in L^{2}(\mathbb{D})$ ).

Next, pick a number $r_{0} \in(0,1)$. For $\theta \in[0,2 \pi]$, we join the point $r_{0} e^{i \theta}$ with $e^{i \theta}$ by a line segment. Then we have

$$
u_{\nu}\left(r_{0} e^{i \theta}\right) \leq \int_{\left[r_{0} e^{i \theta}, e^{i \theta}\right]}|\nabla u| d \mathcal{H}^{1}=\int_{r_{0}}^{1}\left|\nabla u\left(r e^{i \theta}\right)\right| d r .
$$

Integrating over $\theta$ and taking in account that $d \lambda_{2}\left(r e^{i \theta}\right)=r d r d \theta \geq r_{0} d r d \theta$, we get

$$
\int_{\partial \mathcal{B}\left(0, r_{0}\right)} u_{\nu} d \mathcal{H}^{1} \leq \int_{|z| \in\left[r_{0}, 1\right]}|\nabla u(z)| d \lambda_{2}(z) .
$$

The quantity on the right-hand side tends to zero as $r_{0} \rightarrow 1$. It follows that the trace of $u_{\nu}$ on $\partial \mathbb{D}$ (in the sense of the boundary values operator) is equal to zero.

So, $u_{\nu} \in \mathcal{A} d m(\Omega)$, while the sequence $\left\{\left.u_{\nu}\right|_{B_{j}}\right\}_{j=1}^{\infty}$ is finitely supported (because the function $u_{\nu}$ by its definition vanishes on $B_{\nu} \cup B_{\nu+1} \cup \ldots$ ). Let us prove that

$$
C_{I}^{2}(\Omega) \cdot\|u\|_{W^{1,2}(\mathbb{D})}^{2} \geq C_{I}^{2}(\Omega) \cdot\left\|u_{\nu}\right\|_{W^{\circ, 2}(\mathbb{D})}^{2} \geq \sum_{j=1}^{\infty}\left(\left.u_{\nu}\right|_{B_{j}}\right)^{2} .
$$

Indeed, the first inequality follows from estimate (12), which we have already proved; to prove the second inequality, consider the function $\widetilde{u}_{\nu} \in \mathcal{A} d m(\Omega)$ that is equal to $u_{\nu}$ on any set $B_{j}$ and minimizes the norm $\left\|\tilde{u}_{\nu}\right\|_{W^{1,2}(\mathbb{D})}$. Then $\Delta \tilde{u}_{\nu}=0$ in $\Omega$ by the usual variational argument, $\widetilde{u}_{\nu}=u_{\nu}$ on $B_{j}, j=1,2, \ldots$, and $\widetilde{u}_{\nu} \in \mathcal{A} d m(\Omega)$. By Theorem 2.4. the function satisfying these conditions is unique. But the finite linear combination $\widetilde{u}_{\nu}^{\prime}=\sum_{j=1}^{\infty}\left(\left.u_{\nu}\right|_{B_{j}}\right) \cdot \mathfrak{v}_{j}$ also satisfies all conditions mentioned above, so that $\widetilde{u}_{\nu}=\widetilde{u}_{\nu}^{\prime}$. Application of (8) to $\widetilde{u}_{\nu}^{\prime}$ yields

$$
\left\|u_{\nu}\right\|_{W^{1,2}(\mathbb{D})}^{2} \geq\left\|\widetilde{u}_{\nu}\right\|_{W^{1,2}(\mathbb{D})}^{2} \geq C_{I}^{-2}(\Omega) \cdot \sum_{j=1}^{\infty}\left(\left.u_{\nu}\right|_{B_{j}}\right)^{2} .
$$

Inequality (13) is established. Now we are going to show that the values $\left.u_{\nu}\right|_{B_{j}}$ are not very small compared to $\left.u\right|_{B_{j}}$.

Fix a number $n \in \mathbb{N}$ and pick some $\nu>n$; the choice of $\nu$ will be specified below. Suppose that $T \in \mathbb{R}$ and $\Gamma:[0, T] \rightarrow \mathbb{C}$ is a Lipschitz curve joining some point $z_{0} \in B_{n}$ 
with a point on $\partial \mathbb{D}$ or with a point on one of the sets $B_{j}, j=\nu, \nu+1, \ldots$ We need to estimate $L(\Gamma)$. We may assume that $\Gamma([0, T)) \subset \mathbb{D}$. Put $\delta=1-|\Gamma(T)|$. If our curve ends on $\partial \mathbb{D}$, then $\delta=0$. If not, then we may take $\nu$ so large that $\delta$ will be as small as we want, because in this case $\Gamma$ ends at one of the holes $B_{\nu}, B_{\nu+1}, \ldots$, while the holes accumulate only to $\partial \mathbb{D}$. In particular, we may pick $\nu$ so large that $\delta<\operatorname{dist}\left(B_{n}, \partial \mathbb{D}\right)$. For any $t<T$, the arc $\Gamma([0, t])$ lies strictly inside $\mathbb{D}$, therefore $u$ is piecewise-smooth on this arc. Then we may apply the Newton-Leibniz formula to get

$$
u(\Gamma(t))-u(\Gamma(0))=\int_{\Gamma([0, t])}(\nabla u)_{\tau} d \mathcal{H}^{1},
$$

where $(\nabla u)_{\tau}$ is the tangent component of the gradient along $\Gamma$. Thus, for any $t<T$ we get the estimate

$$
L(\Gamma) \geq\left|\left(\left.u\right|_{B_{n}}\right)-u(\Gamma(t))\right| .
$$

Put $a=\inf \{|u(z)|: z \in \Gamma([0, T])\}$. Let $\zeta$ denote the point on $\partial \mathbb{D}$ closest to $\Gamma(T)$. If $r \in\left(\delta, \operatorname{dist}\left(B_{n}, \partial \mathbb{D}\right)\right)$, then the $\operatorname{arc} \beta_{r}=\partial \mathcal{B}(\zeta, r) \cap \mathbb{D}$ lying in $\mathbb{D}$ separates $\Gamma(T)$ from $B_{n}$, and thus, intersects $\Gamma$. For almost every $r \in\left(\delta, \operatorname{dist}\left(B_{n}, \partial \mathbb{D}\right)\right)$ the function $u$ is absolutely continuous on $\beta_{r}$, takes zero values at the endpoints of this arc, and $|u(z)| \geq a$ at some point $z \in \beta_{r}$. Thus,

$$
\int_{\beta_{r}}|\nabla u|^{2} d \mathcal{H}^{1} \geq \frac{a}{\pi r}
$$

for almost every $r$. Integration over $r$ gives

$$
\int_{\mathbb{D}}|\nabla u|^{2} d \lambda_{2} \geq \frac{a}{\pi} \cdot\left(\log \operatorname{dist}\left(B_{n}, \partial \mathbb{D}\right)-\log \delta\right) .
$$

If $n$ is fixed, we can make $\delta$ as small as we need by picking $\nu>n$ sufficiently large, and the last estimate shows that in this case the number $a$ will also become small (independently of the choice of $\Gamma$ ). Estimate (14) now implies that $L(\Gamma) \geq\left|\left(\left.u\right|_{B_{j}}\right)\right|-a$. Since $\left.u_{\nu}\right|_{B_{n}}=$ $\inf L(\Gamma)$ over $\Gamma$ joining $B_{n}$ with $\partial \mathbb{D}$ or with one of the holes $B_{\nu}, B_{\nu+1}, \ldots$, we conclude that $\liminf _{\nu \rightarrow \infty}\left|\left(\left.u_{\nu}\right|_{B_{n}}\right)\right| \geq\left|\left(\left.u\right|_{B_{n}}\right)\right|$.

Now fix some $m \in \mathbb{N}$. Inequality (13) implies, in particular, that

$$
\|u\|_{W^{1,2}(\mathbb{D})}^{2} \geq C_{I}^{-2}(\Omega) \cdot \sum_{n=1}^{m}\left(\left.u_{\nu}\right|_{B_{n}}\right)^{2} .
$$

Passing to $\liminf$ as $\nu \rightarrow \infty$, we conclude that

$$
\|u\|_{W^{1,2}(\mathbb{D})}^{2} \geq C_{I}^{-2}(\Omega) \cdot \sum_{n=1}^{m}\left(\left.u\right|_{B_{n}}\right)^{2} .
$$

Now it remains to pass to the limit as $m \rightarrow \infty$, obtaining the desired inequality

$$
\|u\|_{W^{1,2}(\mathbb{D})}^{2} \geq C_{I}^{-2}(\Omega) \cdot \sum_{n=1}^{\infty}\left(\left.u\right|_{B_{n}}\right)^{2}
$$

for any function $u \in \mathcal{A} d m(\Omega)$ such that $\Delta u=0$ in $\Omega$, and, thus, for arbitrary $u \in$ $\mathcal{A} d m(\Omega)$. The proof is finished.

Corollary 2.11. Let $\Omega_{1}, \Omega_{2}$ be regular domains, and let $\left\{B_{j}^{(1)}\right\}_{j=1}^{\infty},\left\{B_{j}^{(2)}\right\}_{j=1}^{\infty}$ be the bounded connected components of their complements to $\mathbb{R}^{2}$. Suppose that $B_{j}^{(1)} \subset B_{j}^{(2)}$ for any $j=1,2, \ldots$ Then $C_{B}\left(\Omega_{2}\right) \geq C_{B}\left(\Omega_{1}\right)$ and $C_{I}\left(\Omega_{2}\right) \leq C_{I}\left(\Omega_{1}\right)$.

Proof. We may use the proposition proved above, comparing the classes of functions admissible for $\Omega_{1}$ and $\Omega_{2}$. However, we can get a straightforward proof relying only on the definitions of the Bessel and interpolation constants. 
2.5. Capacity conditions. Now we relate our problem to $L^{2}$-capacity. A condenser is a pair $\left(E_{1}, E_{2}\right)$ of two sets on the plane; the sets $E_{1}$ and $E_{2}$ are called plates of this condenser. The following definition of capacity is a rude one, but it suffices for our purposes.

Definition 2.12. Let $E_{1}, E_{2} \subset \mathbb{C}$ be arbitrary sets. The capacity of the condenser formed by the plates $E_{1}$ and $E_{2}$ is the quantity

$$
\begin{array}{r}
\operatorname{Cap}_{2}\left(E_{1}, E_{2}\right):=\inf \left\{\int_{\mathbb{C}}|\nabla u|^{2} d \lambda_{2}: u \in W_{\text {loc }}^{1,2}(\mathbb{C}), u \geq 1 \text { a.e. on } E_{1},\right. \\
\left.u \leq 0 \text { a.e. on } E_{2}\right\} .
\end{array}
$$

It is not hard to prove the following statement.

Proposition 2.13. Let $E_{1}, E_{1}^{\prime}, E_{2}, E_{2}^{\prime}, E_{3} \subset \mathbb{C}$ be arbitrary sets.

(1) The infimum in (15) will not change if, in addition, we require that the functions involved be such that $u(z) \in[0,1]$ for almost every $z \in \mathbb{C}$.

(2) Capacity is symmetric, that is, $\operatorname{Cap}_{2}\left(E_{1}, E_{2}\right)=\operatorname{Cap}_{2}\left(E_{2}, E_{1}\right)$. If $E_{1} \subset E_{1}^{\prime}$, $E_{2} \subset E_{2}^{\prime}$, then $\operatorname{Cap}_{2}\left(E_{1}, E_{2}\right) \leq \operatorname{Cap}_{2}\left(E_{1}^{\prime}, E_{2}^{\prime}\right)$.

(3) Capacity is semiadditive: $\operatorname{Cap}_{2}\left(E_{1}, E_{2} \cup E_{3}\right) \leq \operatorname{Cap}_{2}\left(E_{1}, E_{2}\right)+\operatorname{Cap}_{2}\left(E_{1}, E_{3}\right)$.

Making a conformal change of variables and cutting the singularity of a Möbius transformation, we easily prove the following statement.

Proposition 2.14. Suppose that $E_{1}, E_{2} \subset \mathbb{D}, z_{0} \in \mathbb{D}, \theta \in \mathbb{R}$. Let $\varphi(z)=e^{i \theta} \cdot \frac{z-z_{0}}{1-z \bar{z}_{0}}$, $z \in \mathbb{D}$, be a conformal automorphism of $\mathbb{D}$. Then

$$
\operatorname{Cap}_{2}\left(E_{1}, E_{2}\right)=\operatorname{Cap}_{2}\left(\varphi\left(E_{1}\right), \varphi\left(E_{2}\right)\right) .
$$

Moreover, $\operatorname{Cap}_{2}\left(E, \mathbb{D}^{(c)}\right)=\operatorname{Cap}_{2}\left(\varphi(E), \mathbb{D}^{(c)}\right)$ for any $E \subset \mathbb{D}$.

For any $j=1,2, \ldots$, the function $\mathfrak{v}_{j}$ found in Theorem 2.4 satisfies equations $\left.\mathfrak{v}_{j}\right|_{B_{j}}=1$, $\left.\mathfrak{v}_{j}\right|_{B_{j^{\prime}}}=0, j^{\prime} \neq j$, and minimizes the Dirichlet integral $\int_{\mathbb{C}}|\nabla v|^{2} d \lambda_{2}$ under these conditions (by the construction of that function in the proof of Theorem 2.4). This implies the relation

$$
\left\|\operatorname{Per}_{j}\right\|_{\left(L_{c}^{2,1}(\Omega)\right)^{*}}=\left\|\kappa_{j}\right\|_{L_{c}^{2,1}}(\Omega)=\left\|\mathfrak{v}_{j}\right\|_{W^{1,2}(\mathbb{D})}=\operatorname{Cap}_{2}\left(B_{j}, \Omega^{(c)} \backslash B_{j}\right)^{1 / 2} .
$$

(We denote by $\Omega^{(c)}$ the set $\mathbb{C} \backslash \Omega=\mathbb{D}^{(c)} \cup \bigcup_{j=1}^{\infty} B_{j}$, where $\mathbb{D}^{(c)}=\{z \in \mathbb{C}:|z| \geq 1\}$.) This immediately leads us to the following necessary conditions for the Bessel and interpolation properties.

Proposition 2.15. Let $\Omega$ be regular domain.

(1) We have $\widetilde{C}_{B}^{2}(\Omega)=\sup _{j \in \mathbb{N}} \operatorname{Cap}_{2}\left(B_{j}, \Omega^{(c)} \backslash B_{j}\right)$.

(2) If $\Omega$ possesses the interpolation property, then $\operatorname{Cap}_{2}\left(B_{j}, \Omega^{(c)} \backslash B_{j}\right) \geq C_{I}^{-2}(\Omega)$ for any $j=1,2, \ldots$.

One can easily give examples showing that the interpolation property of $\Omega$ may fail even if the quantities $\operatorname{Cap}_{2}\left(B_{j}, \Omega^{(c)} \backslash B_{j}\right), j=1,2, \ldots$, are bounded from above and separated away from zero uniformly. Namely, let $\delta>0$ be sufficiently small, and put $\Omega_{\delta}=\mathbb{D} \backslash(\overline{\mathcal{B}}(-2 \delta, \delta) \cup \overline{\mathcal{B}}(2 \delta, \delta))$. By Theorem 5.4 proved below, $C_{I}\left(\Omega_{\delta}\right) \stackrel{\delta \rightarrow 0}{\longrightarrow}+\infty$, while the capacities $\operatorname{Cap}_{2}\left(\overline{\mathcal{B}}( \pm 2 \delta, \delta), \mathbb{D}^{(c)} \cup \overline{\mathcal{B}}(\mp 2 \delta, \delta)\right)$ are bounded from above and from below (see Proposition 2.19 and Corollary 2.23 below). Acting with conformal shifts as in Example 3.3 in Subsection 3.3 (see below), from a sequence of such domains with $\delta$ tending to zero one can construct a domain with infinite interpolation constant for which the capacities of the form $\mathrm{Cap}_{2}\left(B_{j}, \Omega^{(c)} \backslash B_{j}\right)$ still satisfy upper and lower estimates.

Nevertheless, the Bessel property and the weak Bessel property turn out to be equivalent. 


\subsection{The weak Bessel property implies the Bessel property.}

Theorem 2.16. If a regular domain $\Omega$ has the weak Bessel property, then it has the Bessel property, moreover, $C_{B}(\Omega) \leq \sqrt{2} \widetilde{C}_{B}(\Omega)$.

Proof. We show that $\left\|\sum_{j=1}^{\infty} a_{j} \kappa_{j}\right\|_{L_{c}^{2,1}(\Omega)} \leq \sqrt{2} \widetilde{C}_{B}(\Omega) \cdot\left\|\left\{a_{j}\right\}_{j=1}^{\infty}\right\|_{\ell^{2}}$ for any finitely supported sequence $\left\{a_{j}\right\}_{j=1}^{\infty}$ (the reproducing kernels $\kappa_{j}$ were found in Theorem 2.4). By Proposition 2.8, this suffices to prove our statement. First, we get an estimate for nonnegative sequences $\left\{a_{j}\right\}_{j=1}^{\infty}$. To this end, we observe that, by Proposition 2.6, we have $\left\langle\kappa_{j}, \kappa_{k}\right\rangle_{L_{c}^{2,1}(\Omega)}<0$ for $j \neq k, j, k=1,2, \ldots$ Hence,

$$
\left\|\sum_{j=1}^{\infty} a_{j} \kappa_{j}\right\|_{L_{c}^{2,1}(\Omega)}^{2}=\sum_{j=1}^{\infty} a_{j}^{2}\left\|\kappa_{j}\right\|_{L_{c}^{2,1}(\Omega)}^{2}+2 \sum_{j>k} a_{j} a_{k}\left\langle\kappa_{j}, \kappa_{k}\right\rangle_{L_{c}^{2,1}(\Omega)} \leq \widetilde{C}_{B}^{2}(\Omega) \cdot\left\|\left\{a_{j}\right\}_{j=1}^{\infty}\right\|_{\ell^{2}}^{2},
$$

because all $a_{j}$ have the same sign and $\left\|\kappa_{j}\right\|_{L_{c}^{2,1}(\Omega)} \leq \widetilde{C}_{B}(\Omega)$ for all $j=1,2, \ldots$ Now the desired estimate for arbitrary, not necessarily nonnegative sequences $\left\{a_{j}\right\}_{j=1}^{\infty}$ follows from the estimate obtained above by subdivision of such a sequence into positive and negative parts.

Remark. Theorem 2.16 can be proved by invoking admissible functions and using Proposition 2.10. Namely, for a finitely supported sequence $\left\{a_{j}\right\}_{j=1}^{\infty}$ of nonnegative numbers put $u(z):=\max _{j \in \mathbb{N}} a_{j} \mathfrak{v}_{j}(z), z \in \mathbb{D}$, where the $\mathfrak{v}_{j}$ are the functions constructed in Theorem 2.4. It is easily seen that $\left.u\right|_{B_{j}}=a_{j}$ for any $j=1,2, \ldots$ and $\|u\|_{W^{1,2}(\mathbb{D})} \leq$ $\widetilde{C}_{B}(\Omega) \cdot\left\|\left\{a_{j}\right\}_{j=1}^{\infty}\right\|_{\ell^{2}}$ (because $\widetilde{C}_{B}(\Omega)=\sup _{j \in \mathbb{N}}\left\|\mathfrak{v}_{j}\right\|_{W^{1,2}(\mathbb{D})}$ ). Such a function $u$ meets all the conditions of the first assertion in Proposition 2.10. Now, for arbitrary (not necessarily nonnegative) sequences $\left\{a_{j}\right\}_{j=1}^{\infty}$, the construction can again be done by subdividing such a sequence into the nonnegative and nonpositive parts.

Remark. Suppose that $H$ is an abstract Hilbert space and that a countable system $\left\{\xi_{j}\right\}_{j=1}^{\infty}$ in $H$ is understood as a system of reproducing kernels in the interpolation problem for the operator $T: H \rightarrow \ell^{2}, T x=\left\{\left\langle x, \xi_{j}\right\rangle_{H}\right\}_{j=1}^{\infty}, x \in H$. Is it true that the weak Bessel property in such a problem implies the Bessel property? Of course, no. Is it true that the interpolation and weak Bessel properties imply the Bessel property? The answer is also in the negative. More precisely, there exists a sequence of vectors $\xi_{1}, \xi_{2}, \ldots$ in the Hilbert space $\ell^{2}$ such that:

(1) $\sup _{j \in \mathbb{N}}\left\|\xi_{j}\right\|_{\ell^{2}}<+\infty$;

(2) $\left\|\sum_{j=1}^{\infty} a_{j} \xi_{j}\right\|_{\ell^{2}} \geq C_{1} \cdot\left\|\left\{a_{j}\right\}_{j=1}^{\infty}\right\|_{\ell^{2}}$ for any finitely supported sequence $\left\{a_{j}\right\}_{j=1}^{\infty}$ with some absolute constant $C_{1}>0$;

(3) a reverse estimate $\left\|\sum_{j=1}^{\infty} a_{j} \xi_{j}\right\|_{\ell^{2}} \leq C_{2} \cdot\left\|\left\{a_{j}\right\}_{j=1}^{\infty}\right\|_{\ell^{2}}$ for finitely supported sequences $\left\{a_{j}\right\}_{j=1}^{\infty}$ fails for any $C_{2}<+\infty$.

Construction of such a sequence deviates from our main exposition and is not given in this paper.

2.7. Hyperbolic metric and metric capacity estimates. Our problem is conformally invariant. Thus, we may expect that the necessary and sufficient conditions will be conformally invariant. Let us recall some notions of hyperbolic geometry (see also [5]).

The hyperbolic metric in the disk is the metric $\frac{2|d z|}{1-|z|^{2}}$. The geodesics in this metric are the arcs of circles (or segments of lines) orthogonal to $\partial \mathbb{D}$. The Möbius automorphisms

$$
z \mapsto e^{i \theta} \cdot \frac{z-z_{0}}{1-z \bar{z}_{0}}, \quad \theta \in \mathbb{R}, \quad z_{0} \in \mathbb{D}
$$


of the disk are isometries in the hyperbolic metric. The following formula for the hyperbolic distance $\operatorname{dist}_{H}$ is well known:

$$
\operatorname{dist}_{H}\left(z_{1}, z_{2}\right)=\operatorname{arctanh}\left|\frac{z_{1}-z_{2}}{1-z_{1} \bar{z}_{2}}\right|, \quad z_{1}, z_{2} \in \mathbb{D} .
$$

Denote by $\mathcal{B}_{H}(z, r)$ the open ball in the hyperbolic metric of radius $r \geq 0$ and centered at a point $z \in \mathbb{D}$. In particular, from formula (17) it follows that $\mathcal{B}_{H}(0,1)=\mathcal{B}(0, \tanh 1)$.

We shall use the hyperbolic diameters of the sets $B_{j}$, which are their diameters in the hyperbolic metric. Denote these quantities by $\operatorname{diam}_{H}\left(B_{j}\right)$ (the $\operatorname{symbol} \operatorname{diam}\left(B_{j}\right)$ stands for the usual Euclidean diameter of $B_{j}$ ).

We point out the relationship between the hyperbolic diameters of sets and their Euclidean metric properties. Let $E$ be a compact set in $\mathbb{D}$, and let $b=\frac{\operatorname{diam} E}{\operatorname{dist}(E, \partial \mathbb{D})}$. It is easy to prove that

$$
2 b \geq \operatorname{diam}_{H}(E) \geq \operatorname{arctanh}\left(\frac{b}{4+b}\right)=\frac{1}{2} \log \left(\frac{b}{2}+1\right) .
$$

This immediately provides the following statements.

Proposition 2.17. Let $\Omega$ be a regular domain.

(1) $\inf _{j \in \mathbb{N}} \operatorname{diam}_{H}\left(B_{j}\right)>0$ if and only if $\inf _{j \in \mathbb{N}} \frac{\operatorname{diam} E}{\operatorname{dist}(E, \partial \mathbb{D})}>0$; any of these two infima can be estimated from below in terms of the other.

(2) $\sup _{j \in \mathbb{N}} \operatorname{diam}_{H}\left(B_{j}\right)<+\infty$ if and only if $\sup _{j \in \mathbb{N}} \frac{\operatorname{diam} E}{\operatorname{dist}(E, \partial \mathbb{D})}<+\infty$; any of these two suprema can be estimated from above in terms of the other.

We make essential use of the following separation conditions for the holes $B_{j}$.

Definition 2.18. Pick $\varepsilon>0$. We say that the holes $B_{j}$ are $\varepsilon$-strongly separated if for any two distinct indices $j$ and $j^{\prime}$ we have

$$
\operatorname{dist}\left(B_{j}, B_{j^{\prime}}\right) \geq \varepsilon \cdot \max \left\{\operatorname{diam} B_{j}, \operatorname{diam} B_{j^{\prime}}\right\} .
$$

If for any two distinct indices $j$ and $j^{\prime}$ we have

$$
\operatorname{dist}\left(B_{j}, B_{j^{\prime}}\right) \geq \varepsilon \cdot \min \left\{\operatorname{diam} B_{j}, \operatorname{diam} B_{j^{\prime}}\right\},
$$

then we say that the holes $B_{j}$ are $\varepsilon$-weakly separated.

We say that the holes are strongly (or weakly) separated if they are $\varepsilon$-strongly (respectively, $\varepsilon$-weakly) separated for some $\varepsilon>0$.

In the case of round holes, the strong separation condition is equivalent to the property that the disks $(1+\varepsilon) B_{j}$ (i.e., the disks with the same centers and $(1+\varepsilon)$ times enlarged radii) are pairwise disjoint for some $\varepsilon>0$. If the numbers $\operatorname{diam}_{H}\left(B_{j}\right)$ for all $j=1,2, \ldots$ do not exceed some given value, then the separation conditions can be reformulated in a Möbius invariant form, that is via the hyperbolic metric. However, it is more convenient for us to work with Euclidean distances.

We shall repeatedly use the following estimate.

Proposition 2.19. Suppose that any of two sets $E_{1}, E_{2} \subset \mathbb{C}$ is either the closure of a domain with $C^{\infty}$-smooth boundary or $\mathbb{D}^{(c)}, E_{1} \cap E_{2}=\varnothing$. Then

$$
\frac{\min \left\{\operatorname{diam} E_{1}, \operatorname{diam} E_{2}\right\}}{\operatorname{dist}\left(E_{1}, E_{2}\right)} \leq \frac{\exp \left(24 \pi \cdot \operatorname{Cap}_{2}\left(E_{1}, E_{2}\right)\right)-1}{\pi} .
$$

The proof is given in the Appendix.

Propositions 2.15, 2.17, and 2.19 immediately imply the next claim.

Proposition 2.20. If a regular domain $\Omega$ possesses the weak Bessel property, then: 
(1) the quantity $\sup _{j \in \mathbb{N}} \operatorname{diam}_{H}\left(B_{j}\right)$ is finite and can be estimated from above via only $\widetilde{C}_{B}(\Omega)$;

(2) the holes $B_{j}$ are $\varepsilon$-weakly separated for some $\varepsilon>0$ depending only on $\widetilde{C}_{B}(\Omega)$.

If the quantities $\operatorname{diam}_{H}\left(B_{j}\right)$ are separated from zero, then we can say more.

Proposition 2.21. Let $\Omega$ be a regular domain possessing the weak Bessel property. If $\operatorname{diam}_{H}\left(B_{j}\right) \geq r$ for all $j=1,2, \ldots$ with some constant $r>0$, then the sets $B_{j}$ are $\varepsilon$-strongly separated with some $\varepsilon>0$ depending only on $r$ and $\widetilde{C}_{B}(\Omega)$.

The proof is given in the Appendix.

Further, we estimate the capacity from above.

Proposition 2.22. Suppose $E \subset \mathbb{C}$ is a bounded set, $\Lambda=\operatorname{diam} E>0, \varepsilon>0$, and let $U$ be the $(\Lambda \varepsilon)$-neighborhood of $E, U^{(c)}=\mathbb{C} \backslash U$. Then $\operatorname{Cap}_{2}\left(E, U^{(c)}\right) \leq \frac{\pi(1+2 \varepsilon)^{2}}{\varepsilon^{2}}$.

The proof is given in the Appendix.

Corollary 2.23. If sets $E_{1}, E_{2} \subset \mathbb{C}$ are bounded and

$$
\operatorname{dist}\left(E_{1}, E_{2}\right) \geq \varepsilon \cdot \min \left\{\operatorname{diam} E_{1}, \operatorname{diam} E_{2}\right\}
$$

for some $\varepsilon>0$, then $\operatorname{Cap}_{2}\left(E_{1}, E_{2}\right) \leq \frac{\pi(1+2 \varepsilon)^{2}}{\varepsilon^{2}}$.

Remark. In accordance with Proposition 2.19 and Corollary 2.23, a condenser formed by connected plates $E_{1}, E_{2}$ looks, from the viewpoint of the upper or lower boundedness of its capacity, like a condenser formed by disks of diameters diam $E_{1}$ and diam $E_{2}$ placed at the distance $\operatorname{dist}\left(E_{1}, E_{2}\right)$ from each other.

Finally, we have the following statement.

Proposition 2.24. Let $\varepsilon$ be a positive number and $\Omega$ a regular domain. If the holes $B_{j}$ are $\varepsilon$-strongly separated and $\sup _{j \in \mathbb{N}} \operatorname{diam}_{H}\left(B_{j}\right)<+\infty$, then $\Omega$ has the Bessel property, and the Bessel constant $C_{B}(\Omega)$ is bounded from above by a quantity depending only on $\varepsilon$ and $\sup _{j \in \mathbb{N}} \operatorname{diam}_{H}\left(B_{j}\right)$.

The proof is given in the Appendix.

\section{§3. Partial COMPlete interpolation CRiterion}

In this section we derive a criterion for complete interpolation in a regular domain $\Omega$ in the case where one of the following conditions is fulfilled: either $\inf _{j \in \mathbb{N}} \operatorname{diam}_{H}\left(B_{j}\right)>0$, or the sets $B_{j}$ are strongly separated. If one of these conditions is satisfied, then complete interpolation is equivalent to the two of them. However, we shall construct an example showing that none of these conditions is necessary for complete interpolation.

The main result of this section, Theorem 3.7, is overlapped by Theorem 5.8, Nevertheless, the patch method and an analog of Carleson measures appearing in this section can be of independent interest.

3.1. A sufficient condition for interpolation. Our goal in this subsection is to prove that if the hyperbolic diameters of holes are bounded from below, then the domain $\Omega$ has the interpolation property. This suffices if the holes are disks. If holes have arbitrary form, then we shall also impose the separation condition.

Let $\Omega$ be a regular domain. We introduce a (positive) measure in $\mathbb{D}$ by putting

$$
\mu_{\Omega}:=\sum_{j=1}^{\infty} \frac{\mathbb{1}_{B_{j}} \cdot \lambda_{2}}{\lambda_{2}\left(B_{j}\right)} .
$$


Next, we shall say that a positive measure $\mu$ in unit disk $\mathbb{D}$ possesses the (MC) property if for any function $u \in \stackrel{\circ}{W}^{1,2}(\mathbb{D})$ we have

$$
\int_{\mathbb{D}}|\nabla u|^{2} d \lambda_{2} \geq c \cdot \int_{\mathbb{D}} u^{2} d \mu
$$

with some $c>0$ independent of $u$. The abbreviation (MC) stands for "Maz'ya-Carleson". The choice of this term is related to the fact that sharp results on embeddings of Sobolev spaces into $L^{p}(\mu)$, where $\mu$ is some measure in $\mathbb{R}^{n}$, can be found in the well-known monograph [8] by V. G. Maz'ya. On the other hand, in classical problems on analytic interpolation, Carleson measures arise in a similar context (see [10]).

We recall that, by Theorem 2.5, the measure $\mu_{0}=\frac{\lambda_{2}}{(1-|z|)^{2}}$ possesses the (MC) property.

Lemma 3.1. Let $\Omega$ be a regular domain. If the measure $\mu_{\Omega}$ given by (18) satisfies the (MC) condition (estimate (19)), then $\Omega$ has the interpolation property, whereas $C_{I}(\Omega)$ can be estimated from above in terms of the constant occurring in the (MC) condition for the measure $\mu_{\Omega}$.

Proof. Observe that $\mu_{\Omega}\left(B_{j}\right)=1$ because the sets $B_{j}$ are disjoint. Thus, by the second condition in Proposition 2.10, the interpolation property of $\Omega$ can be reformulated in the following way:

$$
\int_{\mathbb{D}}|\nabla u|^{2} d \lambda_{2} \geq C_{I}^{-2}(\Omega) \cdot \int_{\mathbb{D}} u^{2} d \mu_{\Omega}
$$

for any admissible function $u \in \mathcal{A} d m(\Omega)$. But if the measure $\mu_{\Omega}$ has the (MC) property, then such an estimate is true even for all $u \in \stackrel{\circ}{W}^{1,2}(\mathbb{D})$.

Now we prove a sufficient interpolation condition for domains with round holes.

Theorem 3.2. Suppose that the holes $B_{j}$ are disks and that the numbers $\operatorname{diam}_{H}\left(B_{j}\right)$ are bounded from the below. Then the domain $\Omega$ has the interpolation property and $C_{I}(\Omega)$ does not exceed some constant depending only on $\inf _{j \in \mathbb{N}} \operatorname{diam}_{H}\left(B_{j}\right)$.

Proof. By Proposition 2.17, $c \cdot \operatorname{diam} B_{j} \geq \operatorname{dist}\left(B_{j}, \partial \mathbb{D}\right)$ with some $c$ depending only on $\inf _{j \in \mathbb{N}} \operatorname{diam}_{H}\left(B_{j}\right)$. Hence, $1-|z| \leq(c+1) \cdot \operatorname{diam} B_{j}$ for each $z \in B_{j}$. We have

$$
\frac{\pi}{4 \lambda_{2}\left(B_{j}\right)}=\frac{1}{\operatorname{diam}^{2} B_{j}} \leq \frac{(c+1)^{2}}{(1-|z|)^{2}}, \quad z \in B_{j},
$$

By the definition of the measures $\mu_{0}$ and $\mu_{\Omega}$, this implies that

$$
\mu_{\Omega} \leq \frac{4(c+1)^{2}}{\pi} \cdot \mu_{0}
$$

Now the (MC) property of the measure $\mu_{\Omega}$ is provided by this property of the measure $\mu_{0}$, and the interpolation property of $\Omega$ is immediate from Lemma 3.1 Moreover, $C_{I}(\Omega)$ can be estimated from above by a constant depending only on $\inf _{j \in \mathbb{N}} \operatorname{diam}_{H}\left(B_{j}\right)$.

In Subsection 3.3. we shall give Example 3.3 to show that the interpolation property (even together with the Bessel property) does not imply the (MC) property of the measure $\mu_{\Omega}$ (even for domains with round holes). In other words, in general, estimate (19) for functions $u$ that are constant on any set $B_{j}$ does not imply such an estimate for arbitrary $u \in \stackrel{\circ}{W}^{1,2}(\mathbb{D})$.

If holes $B_{j}$ are not disks, then estimate (20) in the proof of the last theorem may fail. We give an example showing that for domains with holes of arbitrary form the assertion of Theorem 3.2 is not true indeed. For this, we pick a sufficiently large number $n \in \mathbb{N}$ and cut from $\mathbb{D}$ line segments of the form $\left[\frac{1}{3} \cdot e^{2 \pi i k / n}, \frac{1}{2} \cdot e^{2 \pi i k / n}\right]$ with $k=0,1, \ldots, n-1$. Next, let 
the holes $B_{k}$ with these $k$ be closed $\varepsilon$-neighborhoods of these segments, where $\varepsilon=\varepsilon_{n}>0$ is so small that such neighborhoods are pairwise disjoint. Put $\Omega_{n}=\mathbb{D} \backslash \bigcup_{k=0}^{n-1} B_{k}$. The quantities $\operatorname{diam}_{H} B_{k}$ are separated away from zero by a constant not depending on $n$ (and on $\varepsilon$ ). On the other hand, it is easy to choose a function $u \in \mathscr{W}^{1,2}(\mathbb{D})$ that equals 1 on $\mathcal{B}(0,3 / 4)$. For such a function we have $\sum_{k=0}^{n-1}\left(\left.u\right|_{B_{k}}\right)^{2} \geq n$. By the second assertion of Proposition 2.10, this means that $C_{I}(\Omega) \geq \sqrt{n} \cdot c$ with an absolute constant $c>0$. Acting by conformal shifts like in Example 3.3 in Subsection 3.3 (see below), it is possible to construct a domain $\Omega$ such that the holes in $\Omega$ have hyperbolic diameters bounded from below, but $C_{I}(\Omega)=+\infty$.

Now we prove that the domain $\Omega$ still has the interpolation property if we add the holes separation condition to the estimate $\inf _{j \in \mathbb{N}} \operatorname{diam}_{H}\left(B_{j}\right)>0$. We are mainly interested in the complete interpolation property of $\Omega$; the estimate $\sup _{j \in \mathbb{N}} \operatorname{diam}_{H}\left(B_{j}\right)<+\infty$ and the weak separation of the holes are necessary for that. In the proof of Proposition 2.21, it was established that the estimates $\inf _{j \in \mathbb{N}} \operatorname{diam}_{H}\left(B_{j}\right)>0$ and $\sup _{j \in \mathbb{N}} \operatorname{diam}_{H}\left(B_{j}\right)<+\infty$ together with the weak separation of the holes $B_{j}$ imply their strong separation. This condition is what we add to the estimate

$$
\inf _{j \in \mathbb{N}} \operatorname{diam}_{H}\left(B_{j}\right)>0 .
$$

Theorem 3.3. Suppose that the domain $\Omega$ is regular, the holes $B_{j}$ are $\varepsilon$-strongly separated with some $\varepsilon>0$, and $\inf _{j \in \mathbb{N}} \operatorname{diam}_{H}\left(B_{j}\right)>0$. Then $\Omega$ possesses the interpolation property and the constant $C_{I}(\Omega)$ can be estimated from above in terms of $\varepsilon$ and $\inf _{j \in \mathbb{N}} \operatorname{diam}_{H}\left(B_{j}\right)$.

Proof. We shall not prove the (MC) property of the measure $\mu_{\Omega}$ though it seems to be possible. Instead, we approximate the holes $B_{j}$ by appropriate disks $R_{j}$ and work with these disks like in the preceding theorem.

Let $j=1,2, \ldots$ Put $\Lambda_{j}=\operatorname{diam} B_{j}$ (Euclidean), and denote by $U_{j}$ the $\left(\varepsilon \Lambda_{j} / 2\right)$-neighborhood of the set $B_{j}$. By the hypothesis, the sets $U_{j}$ are pairwise disjoint. We may assume that $\varepsilon<1$. Pick arbitrary $z_{j} \in B_{j}, j=1,2, \ldots$ Put $R_{j}:=\mathcal{B}\left(z_{j}, \varepsilon \Lambda_{j} / 2\right)$ and, for $t \in\left[0, \varepsilon \Lambda_{j} / 2\right]$, define a circumference $\beta_{t}=\partial \mathcal{B}\left(z_{j}, t\right)$ concentric to $R_{j}$. Notice that $\beta_{t} \cap B_{j} \neq \varnothing$ because $z_{j} \in B_{j}$, $\operatorname{diam} B_{j}>\varepsilon \Lambda_{j}$, and $B_{j}$ is connected.

Pick $u \in \mathcal{A} d m(\Omega)$. We are going to check the conditions of the second assertion of Proposition 2.10. Denote by $a_{j}$ the constant value $\left.u\right|_{B_{j}}$. Define the function $u$ to be zero outside of $\mathbb{D}$; now $u$ is defined on any $R_{j}$ even if $R_{j} \not \subset \mathbb{D}$.

Now, we correct the function $u$ on a set of zero measure so that $u$ be absolutely continuous on the circumferences $\beta_{t}$ with almost all radii $t \in\left[0, \varepsilon \Lambda_{j} / 2\right]$. Suppose that $t$ is one of such radii. The function $u$ takes the value $a_{j}$ on $\beta_{t}$. Hence, for any point $z \in \beta_{t}$ we have

$$
\left|a_{j}\right| \leq|u(z)|+\int_{\beta_{t}}|\nabla u| d \mathcal{H}^{1} \leq|u(z)|+\sqrt{2 \pi t}\left(\int_{\beta_{t}}|\nabla u|^{2} d \mathcal{H}^{1}\right)^{1 / 2},
$$

whence

$$
a_{j}^{2} \leq 2 u^{2}(z)+4 \pi t \int_{\beta_{t}}|\nabla u|^{2} d \mathcal{H}^{1} .
$$

Integration in $z \in \beta_{t}$ over $\mathcal{H}^{1}$ gives

$$
2 \pi t a_{j}^{2} \leq 2 \int_{\beta_{t}} u^{2}(z) d \mathcal{H}^{1}+8 \pi^{2} t^{2} \int_{\beta_{t}}|\nabla u|^{2} d \mathcal{H}^{1} .
$$

Now we integrate this inequality with respect to $t \in\left[0, \varepsilon \Lambda_{j} / 2\right]$; since $t \leq \varepsilon \Lambda_{j} / 2$, we get

$$
\frac{\pi \varepsilon^{2} \Lambda_{j}^{2} a_{j}^{2}}{4} \leq 2 \int_{R_{j}} u^{2} d \lambda_{2}+2 \pi^{2} \varepsilon^{2} \Lambda_{j}^{2} \int_{R_{j}}|\nabla u|^{2} d \lambda_{2} .
$$


Finally, we obtain the estimate

$$
a_{j}^{2} \leq \frac{2}{\lambda_{2}\left(R_{j}\right)} \int_{R_{j}} u^{2} d \lambda_{2}+8 \pi \int_{R_{j}}|\nabla u|^{2} d \lambda_{2} .
$$

The disks $R_{j}$ are pairwise disjoint, being situated in disjoint sets $U_{j}$. Summation of the last inequalities over $j$ yields

$$
\sum_{j=1}^{\infty} a_{j}^{2} \leq 2 \int_{\mathbb{C}} u^{2} d \mu+8 \pi \cdot\|u\|_{W^{1,2}(\mathbb{D})}^{2}=2 \int_{\mathbb{D}} u^{2} d \mu+8 \pi \cdot\|u\|_{W^{1,2}(\mathbb{D})}^{2},
$$

where $\mu=\sum_{j=1}^{\infty} \frac{\mathbb{1}_{R_{j}} \cdot \lambda_{2}}{\lambda_{2}\left(R_{j}\right)}$ is a measure on $\mathbb{C}$; in the last equation we shrank the integration domain from $\mathbb{C}$ to $\mathbb{D}$ because $u=0$ in $\mathbb{D}^{(c)}$ almost everywhere. (Recall that in our construction the disks $R_{j}$ may fail to lie in $\mathbb{D}$.)

The hypothesis of the theorem implies the existence of a constant $c_{1}<+\infty$ (depending only on $\left.\inf _{j \in \mathbb{N}} \operatorname{diam}_{H}\left(B_{j}\right)\right)$ such that

$$
\frac{\operatorname{dist}\left(B_{j}, \partial \mathbb{D}\right)}{\Lambda_{j}} \leq c_{1}
$$

for all $j=1,2, \ldots$ Then $\operatorname{dist}\left(z_{j}, \partial \mathbb{D}\right) \leq \operatorname{diam} B_{j}+\operatorname{dist}\left(B_{j}, \partial \mathbb{D}\right) \leq\left(1+c_{1}\right) \Lambda_{j}$. For any point $z \in R_{j} \cap \mathbb{D}$ we have $1-|z| \leq\left(1-\left|z_{j}\right|\right)+\left|z-z_{j}\right| \leq\left(1+c_{1}+\varepsilon / 2\right) \Lambda_{j}$, whence

$$
\frac{1}{(1-|z|)^{2}} \geq \frac{1}{\left(\left(1+c_{1}+\varepsilon / 2\right) \Lambda_{j}\right)^{2}}=\frac{\pi \varepsilon^{2}}{4\left(1+c_{1}+\varepsilon / 2\right)^{2}} \cdot \frac{1}{\lambda_{2}\left(R_{j}\right)} .
$$

Thus, $\mathbb{1}_{\mathbb{D}} \cdot \mu \leq \frac{4\left(1+c_{1}+2 \varepsilon\right)^{2}}{\pi \varepsilon^{2}} \cdot \mu_{0}$. Now, the (MC) property of the measure $\mu_{0}$ (Theorem 2.5) implies that $\int_{\mathbb{D}} u^{2} d \mu \leq C \cdot\|u\|_{W^{1,2}(\mathbb{D})}^{2}$, where the constant $C>0$ depends only on the constants in the statement of the theorem. From this and (21) we finally conclude that

$$
\sum_{j=1}^{\infty} a_{j}^{2} \leq(8 \pi+2 C) \cdot\|u\|_{W^{1,2}(\mathbb{D})}^{2} .
$$

Here the function $u \in \mathcal{A} d m(\Omega)$ is arbitrary and $a_{j}=\left.u\right|_{B_{j}}$. So, we have checked the assumptions of the second assertion of Proposition 2.10, Theorem 3.3 is proved.

3.2. Lower estimate for $\operatorname{diam}_{H}\left(B_{j}\right)$. In the previous subsection we proved that the condition $\inf \left\{\operatorname{diam}_{H}\left(B_{j}\right): j=1,2, \ldots\right\}>0$ is sufficient for interpolation (in the case of holes of an arbitrary form we should impose the strong separation condition). Now we are going to prove that if the holes are strongly separated, then the interpolation property implies that $\inf \left\{\operatorname{diam}_{H}\left(B_{j}\right): j=1,2, \ldots\right\}>0$. The proof is given only for domains with round holes. Our argument involves the patch method.

Theorem 3.4. Let $\Omega$ be a domain with round holes. Suppose that the $B_{j}$ are $\varepsilon$-strongly separated for some $\varepsilon>0$ and, moreover, that $\sup _{j \in \mathbb{N}} \operatorname{diam}_{H}\left(B_{j}\right)<+\infty$.

If $\Omega$ possesses the interpolation property, then $\inf _{j \in \mathbb{N}} \operatorname{diam}_{H}\left(B_{j}\right)$ is positive and can be estimated from below only in terms of $\varepsilon, \sup _{j \in \mathbb{N}} \operatorname{diam}_{H}\left(B_{j}\right)$, and $C_{I}(\Omega)$.

Proof. If $B \subset \mathbb{C}$ is an open or closed disk centered at some point $z \in \mathbb{C}$ and of radius $r>0$ and if $a>0$, then we denote by $a B$ respectively open or closed disk centered at $z$ and of radius $a r$.

By Proposition 2.17 there exists $\varepsilon_{0}>0$ depending only on $\sup _{j \in \mathbb{N}} \operatorname{diam}_{H}\left(B_{j}\right)$ and such that $\operatorname{dist}\left(B_{j}, \partial \mathbb{D}\right) \geq \varepsilon_{0} \cdot \operatorname{diam} B_{j}$ for all $j=1,2, \ldots$ Reducing $\varepsilon_{0}$ (if necessary), we may assume that $\varepsilon_{0}<\varepsilon / 2$. By the choice of $\varepsilon_{0}$, the disks $\left(1+\varepsilon_{0}\right) B_{j}$ are pairwise disjoint and lie in $\mathbb{D}$ for all $j=1,2, \ldots$. 
We must prove that the hyperbolic diameter of any hole is bounded from below. Let us prove this for one fixed hole, assuming that this disk is $B_{1}$. By the use of a conformal automorphism of the disk $\mathbb{D}$, we reduce the question to the case where $B_{1}=\overline{\mathcal{B}}(0, r)$. (It is not hard to show that the strong separation property is preserved under the Möbuis transformations provided $\sup _{j \in \mathbb{N}} \operatorname{diam}_{H}\left(B_{j}\right)<+\infty$.) Then we need to prove that the radius $r$ is bounded from below.

As a heuristic consideration, assume that there is one hole in the domain. Then the solution of the Dirichlet problem (7) is the function

$$
u_{1}(z)=\left\{\begin{array}{cl}
\frac{\log |z|}{\log r}, & |z|>r ; \\
1, & |z| \leq r
\end{array} \quad(z \in \mathbb{D})\right.
$$

Calculation of the Dirichlet integral of this function shows that the norm $\left\|u_{1}\right\|_{W^{1,2}(\mathbb{D})}$ is small if $r$ is small, that is, the function $u_{1}$ does not satisfy the second condition of Proposition 2.10. If there are other holes in $\Omega$, then $u_{1}$ will still be not admissible for $\Omega$. The idea of the patch method in our proof is to correct the function $u_{1}$ on the sets $\left(1+\varepsilon_{0}\right) B_{j}$ for all $j=1,2, \ldots$ so that it become constant on $B_{j}$; such a function will be admissible for $\Omega$. Thus, the function $u_{1}$ will be perturbed by certain corrections, and we shall need upper estimates for the Dirichlet integral of the sum of these perturbations.

It is convenient to use cylindrical coordinates in order to compute the corrections in question. Let $\mathrm{Cyl}_{\mathrm{r}}$ denote the cylinder of height $|\log r|$ built over the circumference of radius 1 . We introduce coordinates on $\mathrm{Cyl}_{\mathrm{r}}$ : the angle $\theta$ along the circle and the height $y \in(0,|\log r|)$. The coordinates $(\theta, y)$ determine a family of local charts on $\mathrm{Cyl}_{\mathrm{r}}$, which endows $\mathrm{Cyl}_{\mathrm{r}}$ with a conformal structure.

We map the annulus $\mathbb{D} \backslash B_{1}$ onto $\mathrm{Cyl}_{\mathrm{r}}$ by putting

$$
\varphi(z):=(\arg z,-\log |z|), \quad z \in \mathbb{D} \backslash B_{1} .
$$

Then $\varphi$ is a conformal mapping. In particular, it preserves the property to be a harmonic function. Define a function $\widetilde{u}_{1}: \mathrm{Cyl}_{\mathrm{r}} \rightarrow \mathbb{R}$ as $\widetilde{u}_{1}=u_{1} \circ \varphi^{-1}$, this is the push-forward of $u_{1}$ to the cylinder. Notice that $u_{1}(\theta, y)=y /|\log r|$.

The images $\varphi\left(B_{j}\right)$ are sets in $\mathrm{Cyl}_{\mathrm{r}}$ with smooth boundaries. When doing estimates on the cylinder, it is convenient to assume that these images are squares $Q_{j}^{\prime}$ on the cylinder such that their doublings $2 Q_{j}^{\prime}$ are pairwise disjoint (see Figure 6). The images of disks will not, of course, have right angles, but we shall achieve that by shrinking holes on the cylinder. A good separation of the squares $Q_{j}^{\prime}$ will be attained by applying to $\Omega$ a quasiconformal mapping with controlled distortion coefficient and then using Proposition 1.3 on quasiconformal quasiinvariance. The class of quasiconformal mappings is less rigid than that of conformal mappings and allows us to do such technical transitions. A quasiconformal diffeomorphism will be applied in every set $\left(1+\varepsilon_{0}\right) B_{j}$.

We pick a sufficiently large absolute constant $c_{0} \in[1,+\infty$ ) (depending neither on $\varepsilon_{0}$ nor on $C_{I}(\Omega)$ ); the choice of this constant will be clear after the calculation of the asymptotics of elementary functions in the proof of Lemma 3.6.

Lemma 3.5. Suppose that the disks $\left(1+\varepsilon_{0}\right) B_{j}$ are pairwise disjoint and lie in $\mathbb{D}$ for all $j=1,2, \ldots$, and that $c_{0} \geq 1$ is some number. Then there exists a quasiconformal diffeomorphism $\psi: \mathbb{D} \rightarrow \mathbb{D}$ mapping the disks $B_{j}$ to the disks $\psi\left(B_{j}\right)$ and such that $c_{0}^{2} \psi\left(B_{j}\right) \subset B_{j}$ for all $j=1,2, \ldots$ The diffeomorphism $\psi$ can be chosen so that its distortion coefficient $K(\psi)$ be bounded from above by a quantity depending only on $\varepsilon_{0}$ and $c_{0}$.

In other words, the holes in the domain $\psi(\Omega)$ will be disks strongly separated with a sufficiently large separation constant. 
Proof. For $z \in \mathbb{D} \backslash \bigcup_{j=1}^{\infty}\left(1+\varepsilon_{0}\right) B_{j}$, put $\psi(z)=z$. Let $B_{j}=\overline{\mathcal{B}}\left(z_{j}, r_{j}\right)$. In the disk $\left(1+\varepsilon_{0}\right) B_{j}$ we define $\psi$ radially:

$$
\psi\left(z_{j}+R e^{i \theta}\right):=z_{j}+f_{j}(R) e^{i \theta}, \quad \theta \in[0,2 \pi], \quad R \in\left[0,\left(1+\varepsilon_{0}\right) r_{j}\right],
$$

where $f_{j}$ is a smooth monotone increasing bijection of $\left[0,\left(1+\varepsilon_{0}\right) r_{j}\right]$ onto $\left[0,\left(1+\varepsilon_{0}\right) r_{j}\right]$ and $f_{j}\left(r_{j}\right)=r_{j} c_{0}^{-2}$ (recall that $c_{0} \geq 1$ by assumption); we may also assume that $f$ is linear on $\left[0, r_{j}\right]$. In order to have smoothness of $\psi$ on $\partial\left(\left(1+\varepsilon_{0}\right) B_{j}\right)$, we should also require that $f_{j}^{(l)} v\left(\left(1+\varepsilon_{0}\right) r_{j}\right)=0$ for all $l=1,2, \ldots$

Our construction is scale-invariant: namely, we may assume that $f_{j}(t)=r_{j} f_{0}\left(t / r_{j}\right)$ for some fixed function $f_{0}:\left[0,1+\varepsilon_{0}\right] \rightarrow\left[0,1+\varepsilon_{0}\right]$ depending only on $\varepsilon_{0}$ and $c_{0}$ but not on $r_{j}$. Since scalings do not change the distortion coefficient, $K\left(\left.\psi\right|_{\left(1+\varepsilon_{0}\right) B_{j}}\right)$ does not depend on $r_{j}$ but only on the choice of $f_{0}$. By construction, $\psi$ will be a diffeomorphism of $\left(1+\varepsilon_{0}\right) B_{j}$ onto itself up to the boundary $\partial\left(1+\varepsilon_{0}\right) B_{j}$, where $\psi$ is identical. This implies that $\psi: \mathbb{D} \rightarrow \mathbb{D}$ is a quasiconformal diffeomorphism and its distortion coefficient $K(\psi)$ can be estimated from above in terms of $\varepsilon$ and $c_{0}$ only. For any $j=1,2, \ldots$, the set $\psi\left(B_{j}\right)$ is a disk, whereas the concentric disk $c_{0}^{2} \psi\left(B_{j}\right)$ coincides with $B_{j}$ by construction (because $\left.f_{j}\left(r_{j}\right)=r_{j} c_{0}^{-2}\right)$. The lemma is proved.

Let $\psi$ be the diffeomorphism constructed above. By Proposition 1.3. $C_{I}(\psi(\Omega)) \leq$ $\sqrt{K(\psi)} \cdot C_{I}(\Omega)$, where the distortion coefficient $K(\psi)$ depends only on $\varepsilon_{0}$ (that is, on the constants $\varepsilon$ and $\sup _{j \in \mathbb{N}} \operatorname{diam}_{H}\left(B_{j}\right)$ from the hypothesis of the theorem) and on the absolute constant $c_{0}$. Moreover, the hyperbolic diameters of the holes in $\psi(\Omega)$ do not exceed those of the corresponding holes in $\Omega$. Hence, we may prove our theorem under the assumption that the disks $c_{0}^{2} B_{j}$ are pairwise disjoint and lie in $\mathbb{D}$ - otherwise we may pass to the domain $\psi(\Omega)$.

Now we continue the proof of the theorem under the above-mentioned assumption on the good separation of the holes. Recall that we want to get a lower estimate for the radius $r$ of the hole $B_{1}=\overline{\mathcal{B}}(0, r)$, and that the conformal mapping $\varphi: \mathbb{D} \backslash B_{1} \rightarrow \mathrm{Cyl}_{\mathrm{r}}$ is defined by (22).

Let $\Lambda \in[0, \min (\pi,|\log r| / 2)), \theta_{0} \in[0,2 \pi], y_{0} \in(\Lambda,|\log r|-\Lambda)$. A square on the cylinder $\mathrm{Cyl}_{\mathrm{r}}$ (centered at $\left(\theta_{0}, y_{0}\right)$ and of side $2 \Lambda$ ) is the set $Q=\left\{(\theta, y):\left|\theta-\theta_{0}\right| \leq \Lambda\right.$, $\left.\left|y-y_{j}\right| \leq \Lambda\right\}$. If, moreover, $\Lambda<\min (\pi / 2,|\log r| / 4)$ and $y_{0} \in(2 \Lambda,|\log r|-2 \Lambda)$, then the doubling of such a square $Q$ is the set $2 Q:=\left\{(\theta, y):\left|\theta-\theta_{0}\right| \leq 2 \Lambda,\left|y-y_{j}\right| \leq 2 \Lambda\right\}$.

Lemma 3.6. Suppose that an absolute constant $c_{0} \geq 1$ is sufficiently large and that the round holes $B_{j}$ in a regular domain $\Omega$ are such that the sets $c_{0}^{2} B_{j}, j=1,2, \ldots$, are pairwise disjoint and lie in $\mathbb{D}$. Then for $j=2,3, \ldots$ there exist squares $Q_{j}^{\prime}$ on the cylinder $\mathrm{Cyl}_{\mathrm{r}}$ such that

$$
\varphi\left(B_{j}\right) \subset Q_{j}^{\prime} \subset 2 Q_{j}^{\prime} \subset \varphi\left(c_{0}^{2} B_{j}\right) .
$$

Proof. Consider the disk $B_{j}=\overline{\mathcal{B}}\left(z_{j}, r_{j}\right), j \neq 1$. We have $B_{j} \subset c_{0} B_{j} \subset c_{0}^{2} B_{j}$, while the disk $c_{0}^{2} B_{j}$ does not contain the origin - because $0 \in B_{1}$ and the disks $c_{0}^{2} B_{j}$ are disjoint. Let us do the planimetric construction given in Figure 3 and relying on the fact that $0 \notin c_{0} B_{j}$.

Since $0 \notin B_{j}$, there exist two lines passing through 0 and touching the circle $\partial B_{j}$; also, there exist two circles centered at the origin and touching $\partial B_{j}$. Denote the intersection point of these tangent lines and circles by $A_{1}, A_{2}, A_{3}$, and $A_{4}$, as in Figure 3 . Let $Q_{j}$ be the curved quadrilateral circumscribed around the circle $\partial B_{j}$ and having vertices $A_{1}, A_{2}, A_{3}, A_{4}$ and bounded by the arcs $\overline{A_{1} A_{2}}, \overline{A_{3} A_{4}}$ and the segments $A_{2} A_{3}, A_{4} A_{1}$. Then $\varphi\left(Q_{j}\right)$ is a rectangle on $\mathrm{Cyl}_{\mathrm{r}}$.

Denote by $F_{1}$ the point of touching of the line $0 A_{2} A_{3}$ and the circle $\partial B_{j}$ and by $F_{3}$ and $F_{2}$ the point of touching of the arcs $\overline{A_{1} A_{2}}$ and $\overline{A_{3} A_{4}}$ with $\partial B_{j}$, respectively. 


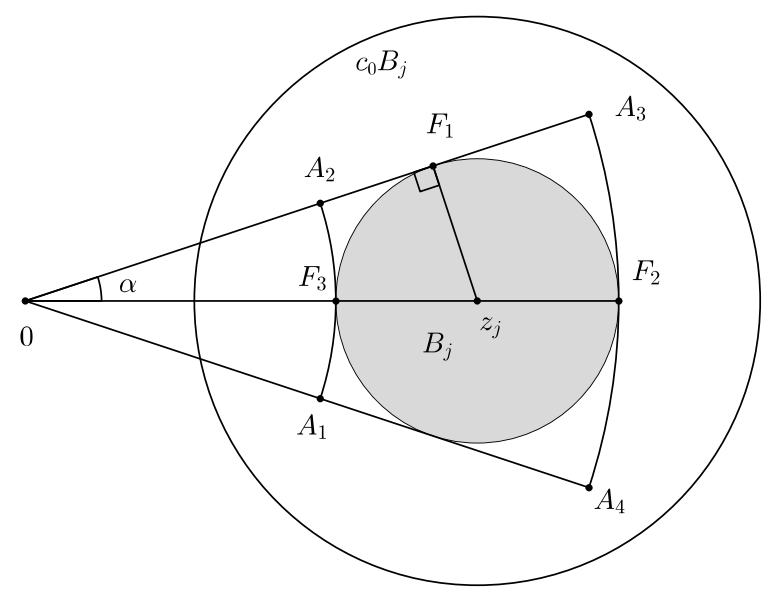

Figure 3. Neighborhood of the hole $B_{j}$.

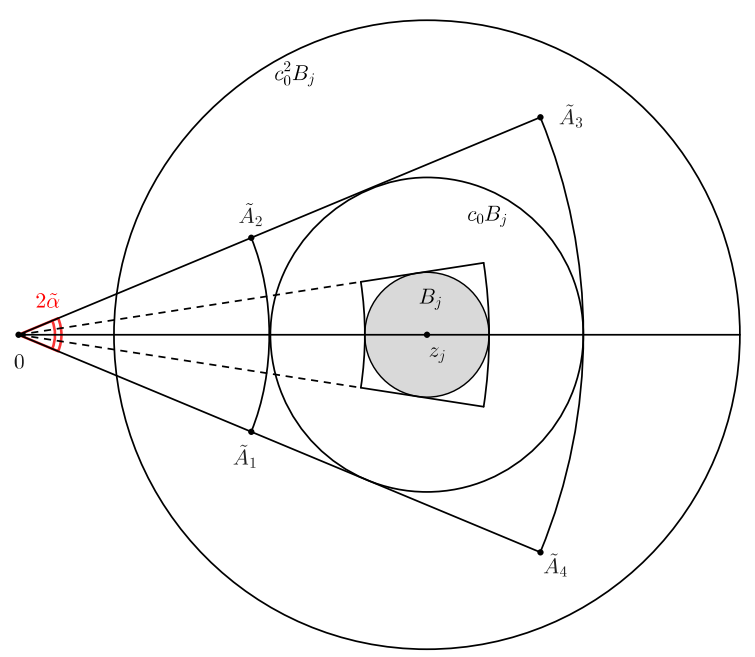

Figure 4. Two neighborhoods of the hole $B_{j}$.

Let $x=\left|z_{j}\right|$. Our draft is scale-invariant, so that all the ratios and all the angles on this draft depend only on $r_{j} / x$. At the same time, $r_{j} / x \leq c_{0}^{-2}$ (because $0 \notin c_{0}^{2} B_{j}$ ). We prove that $Q_{j} \subset c_{0} B_{j}$ if $c_{0}$ is sufficiently large. Applying a rotation if necessary, we may assume that the ray $0 F_{3} z_{j} F_{2}$ is the semiaxis $[0,+\infty)$.

Observe that the angle $\alpha:=\angle A_{2} 0 F_{3}$ is equal to $\arcsin \left(r_{j} / x\right)$. Let $z \in Q_{j}$. Then $|\arg z| \leq \alpha,|z| \in\left[x-r_{j}, x+r_{j}\right]$. Hence, the length of the arc of the circle centered at the origin and joining $z$ with a point on the real axis (more precisely, on the segment $\left[F_{2} F_{3}\right]$ ) does not exceed $\left(x+r_{j}\right) \arcsin \left(r_{j} / x\right)$ and thus is at most

$$
\frac{\pi}{2} \cdot \frac{r_{j}}{x} \cdot\left(x+r_{j}\right) \leq \pi r_{j},
$$

because $x \geq r_{j}$. All the points on the segment $F_{2} F_{3}$ lie in $B_{j}$. Thus, $\left|z-z_{j}\right| \leq(\pi+1) r_{j}$ for any $z \in Q_{j}$. Hence, we have proved that $Q_{j} \subset c_{0} B_{j}$ if $c_{0}>\pi+1$. 


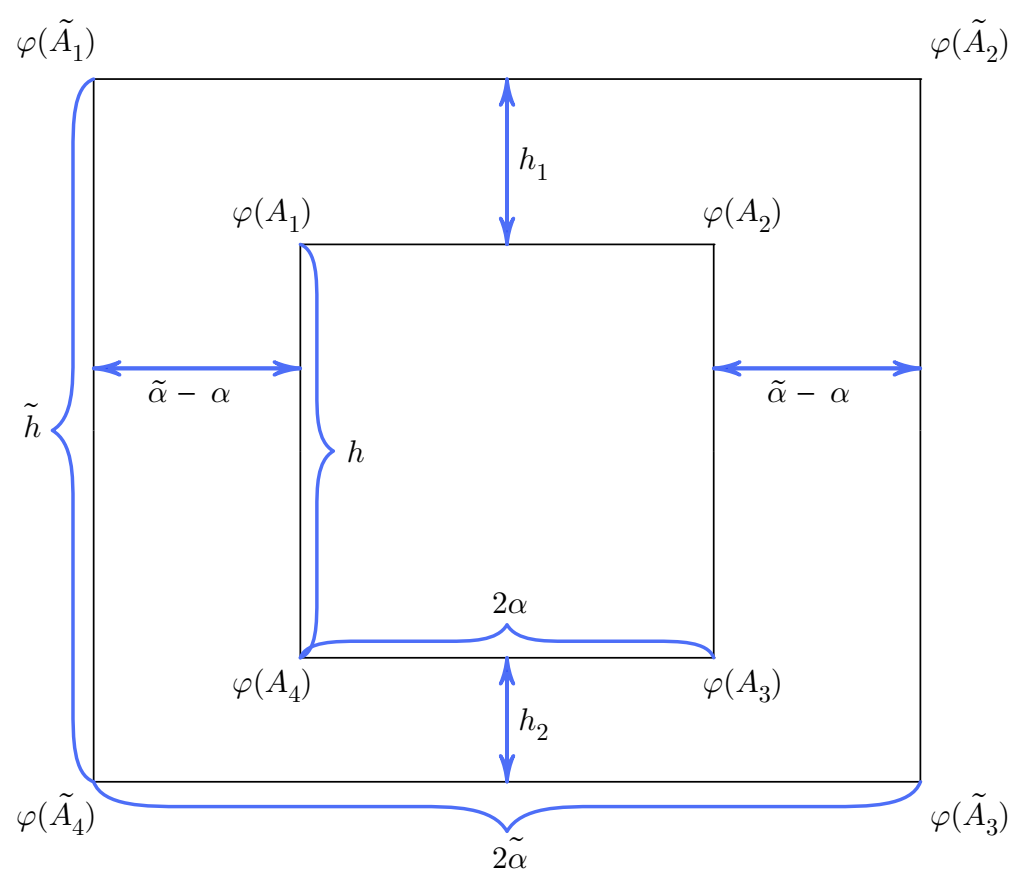

Figure 5. Images of neighborhoods of the hole $B_{j}$.

Now observe that the disk $c_{0}^{2} B_{j}$ does not contain the origin. Thus, we can perform a similar construction for the disk $c_{0} B_{j}$ by circumscribing around it a curved quadrilateral $\widetilde{Q}_{j}$ such that $\varphi\left(\widetilde{Q}_{j}\right)$ is a rectangle on $\mathrm{Cyl}_{\mathrm{r}}$. The construction is given in Figure 4, Namely, this time we consider two lines tangent to $\partial\left(c_{0} B_{j}\right)$ and passing through 0 and also two circles centered at the origin and also tangent to $\partial\left(c_{0} B_{j}\right)$. These four curves bound a curved quadrilateral $\widetilde{Q}_{j}$ formed by the arcs $\widetilde{\widetilde{A}_{1} \widetilde{A}_{2}}$ and $\widetilde{\widetilde{A}_{3} \widetilde{A}_{4}}$ and also by the segments $\widetilde{A}_{2} \widetilde{A}_{3}$ and $\widetilde{A}_{1} \widetilde{A}_{4}$. By $\widetilde{\alpha}$ we denote the half of the angle under which the disk $c_{0} B_{j}$ is seen from the point 0 . Notice that $\widetilde{\alpha}=\arcsin \left(c_{0} r_{j} / x\right)$, like in the previous construction. As above, we prove that $\widetilde{Q}_{j} \subset c_{0}^{2} B_{j}$ if $c_{0}$ is sufficiently large.

Under the mapping $\varphi$, the quadrilaterals $Q_{j}$ and $\widetilde{Q}_{j}$ pass to rectangles on the cylinder (that is, rectangles in one of the charts $(\theta, y)$ ), and the geometric parameters of their mutual position are the quantities $\alpha, \widetilde{\alpha}$ already introduced and also $h, \widetilde{h}, h_{1}, h_{2}$ pointed out in Figure 5. All these parameters depend only on $r_{j} / x$. Moreover, if $c_{0}>2$, then for $x>c_{0}^{2} r_{j}$ we have the following asymptotic estimates (the constants of comparability are absolute and do not depend on $c_{0}$ for $c_{0}>2$ ):

(1) $2 \alpha=2 \arcsin \left(r_{j} / x\right) \asymp r_{j} / x$ and $h=\log \left(\frac{x+r_{j}}{x-r_{j}}\right) \asymp r_{j} / x$;

(2) $\tilde{\alpha}-\alpha=\arcsin \left(c_{0} r_{j} / x\right)-\arcsin \left(r_{j} / x\right) \asymp\left(c_{0}-1\right) r_{j} / x$;

(3) $h_{1}=\log \left(x-r_{j}\right)-\log \left(x-c_{0} r_{j}\right) \asymp\left(c_{0}-1\right) r_{j} / x$;

(4) $h_{2}=\log \left(x+c_{0} r_{j}\right)-\log \left(x+r_{j}\right) \asymp\left(c_{0}-1\right) r_{j} / x$.

If the factor $c_{0}-1$ in the last three estimates is sufficiently large (on account of the comparability constants in all four estimates), then the distances from $\varphi\left(Q_{j}\right)$ to $\partial \varphi\left(\widetilde{Q}_{j}\right)$ are sufficiently large with respect to the lengths of the sides of the rectangle $\varphi\left(Q_{j}\right)$. In this case for the role of $Q_{j}^{\prime}$ we may take any square with side $\max \{2 \alpha, h\}$ containing $\varphi\left(Q_{j}\right)$. The lemma is proved. 


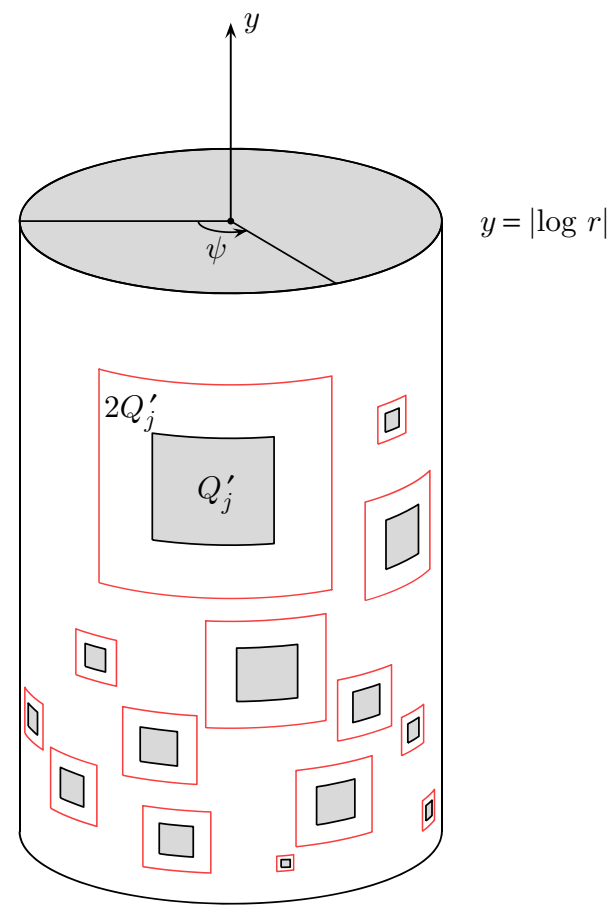

Figure 6. Cylinder with square holes.

Now we continue the proof of Theorem 3.4. We shall correct the function $\widetilde{u}_{1}=u_{1} \circ \varphi^{-1}$ on the cylinder by changing its values on the squares $2 Q_{j}^{\prime}$ constructed in Lemma 3.6. Namely, for every $j=2,3, \ldots$ we find a smooth function $v_{j}: 2 Q_{j}^{\prime} \rightarrow \mathbb{R}$ with the following properties:

(1) $v_{j}=\widetilde{u}_{1}$ on $\partial\left(2 Q_{j}^{\prime}\right)$;

(2) $v_{j} \equiv a_{j}=$ const on $Q_{j}$ with constants $a_{j} \in \mathbb{R}$ as we prescribe.

Moreover, we need that the Dirichlet integrals $\int_{2 Q_{j}^{\prime}}\left|\nabla v_{j}\right|^{2} d \theta d y$ admit a good estimate. For this, it is natural to require that $\Delta v_{j}=0$ in $2 Q_{j}^{\prime} \backslash Q_{j}$ and that the $a_{j}$ be such that the Dirichlet integral of the solution of the corresponding Dirichlet problem will be minimal over all $a_{j} \in \mathbb{R}$. However, we shall avoid such a boundary problem.

Observe - and this is the keypoint of the current proof! - that $\widetilde{u}_{1}(\theta, y)=y /|\log r|$ is a linear function. Thus, the problems on finding the functions $v_{j}$ for different $j$ are obtained from each other by a linear change of variables in the domain of arguments and in the domain of values (together with boundary data).

On the plane $(\theta, y)$, we pick a smooth function $v_{0}: Q_{0}=[-1,1] \times[-1,1] \rightarrow \mathbb{R}$ such that $v_{0}(\theta, y)=y$ for $(\theta, y) \in \partial Q_{0}$ and $v_{0}=0$ on $\frac{1}{2} Q_{0}:=\left[-\frac{1}{2}, \frac{1}{2}\right] \times\left[-\frac{1}{2}, \frac{1}{2}\right]$. The choice of $v_{0}$ depends on nothing. Let $Q_{j}^{\prime}$ be the square with side $\Lambda_{j}$ centered at $\left(\theta_{j}, y_{j}\right) \in \mathrm{Cyl}_{\mathrm{r}}$. Put

$$
v_{j}(\theta, y):=\frac{\Lambda_{j}}{|\log r|} v_{0}\left(\frac{\theta-\theta_{j}}{\Lambda_{j}}, \frac{y-y_{j}}{\Lambda_{j}}\right)+\frac{y_{j}}{|\log r|}, \quad(\theta, y) \in 2 Q_{j}^{\prime} .
$$

In is easily seen that $v_{j}=\widetilde{u}_{1}$ on $\partial\left(2 Q_{j}^{\prime}\right)$, moreover, $v_{j} \equiv y_{j} /|\log r|$ on $Q_{j}^{\prime}$.

Put

$$
\widetilde{u}(\theta, y)= \begin{cases}v_{j}(\theta, y), & (\theta, y) \in 2 Q_{j}^{\prime}, j=2,3, \ldots ; \\ \widetilde{u}_{1}(\theta, y), & (\theta, y) \in \mathrm{Cyl}_{\mathrm{r}} \backslash \bigcup_{j=2}^{\infty} 2 Q_{j}^{\prime} .\end{cases}
$$


Consider the pull-back $u=\widetilde{u} \circ \varphi$ of the function $\tilde{u}$ from $\mathrm{Cyl}_{\mathrm{r}}$ to $\Omega \backslash B_{1}$ and define this function to be equal to 1 on $B_{1}$. We show that $u \in \mathcal{A} d m(\Omega)$. On the compact subsets in $\mathbb{D} \backslash B_{1}$, the function $u$ is piecewise-smooth and continuous, because so is the function $\tilde{u}$ on $\mathrm{Cyl}_{\mathrm{r}}$; moreover, $u$ is continuous up on $\partial B_{1}$. Thus, $u \in W_{\text {loc }}^{1,2}(\mathbb{D})$. We estimate the Dirichlet integral of $u$. Since $u \equiv 1$ on $B_{1}$, we have $\int_{\mathbb{D}}|\nabla u|^{2} d \lambda_{2}=\int_{\mathrm{Cyl}_{\mathrm{r}}}|\nabla \widetilde{u}|^{2} d \theta d y$ by the conformal invariance of the Dirichlet integral (the gradient $\nabla \widetilde{u}$ is taken with respect to $\theta$ and $y)$. We have

$$
\begin{aligned}
\int_{\mathrm{Cyl}_{\mathrm{r}}}|\nabla \widetilde{u}|^{2} d \theta d y & \leq \int_{\mathrm{Cyl}_{\mathrm{r}}}\left|\nabla \tilde{u}_{1}\right|^{2} d \theta d y+\sum_{j=2}^{\infty} \int_{2 Q_{j}^{\prime}}\left|\nabla v_{j}\right|^{2} d \theta d y \\
& =\frac{2 \pi}{|\log r|}+\sum_{j=2}^{\infty} \frac{\Lambda_{j}^{2}}{|\log r|^{2}} \cdot \int_{Q_{0}}\left|\nabla v_{0}\right|^{2} d \theta d y
\end{aligned}
$$

(the last identity is true by the invariance of the Dirichlet integral under scalings). Now observe that the area of $\mathrm{Cyl}_{\mathrm{r}}$ equals $2 \pi|\log r|$. The squares $Q_{j}^{\prime}$ are disjoint, so that their total area $\sum_{j=2}^{\infty} \Lambda_{j}^{2}$ does not exceed $2 \pi|\log r|$. Thus,

$$
\int_{\mathrm{Cyl}_{\mathrm{r}}}|\nabla \tilde{u}|^{2} d \theta d y \leq \frac{C}{|\log r|},
$$

with an absolute constant $C>0$ (it depends only on the choice of $v_{0}$ ). The convergence of the series $\sum_{j=2}^{\infty} \Lambda_{j}^{2}$ implies that $\Lambda_{j} \stackrel{j \rightarrow+\infty}{\longrightarrow} 0$. From the construction of $\widetilde{u}$ we immediately see that $u$ is continuous up to $\partial \mathbb{D}$ and that the boundary values of $u$ on $\partial \mathbb{D}$ are zero. Hence, $u \in \stackrel{\circ}{W}^{1,2}(\mathbb{D})$.

So, the function $u=\widetilde{u} \circ \varphi$ is admissible for $\Omega$ and takes the value 1 on $B_{1}$ and the value $y_{j} /|\log r|$ on $B_{j}, j=2,3, \ldots$ (because $\varphi\left(B_{j}\right) \subset Q_{j}^{\prime}$ ). If the domain $\Omega$ possesses the interpolation property then, by the second assertion of Proposition 2.10, we have

$$
\frac{C}{|\log r|} \geq C_{I}^{-2}(\Omega) \cdot\left(1+\sum_{j=2}^{\infty} \frac{y_{j}^{2}}{|\log r|^{2}}\right),
$$

whence we see that $r$ is bounded from below by a quantity depending only on $C_{I}(\Omega)$, $\sup _{j \in \mathbb{N}} \operatorname{diam}_{H}\left(B_{j}\right)$ and $\varepsilon$ (the dependence on $\sup _{j \in \mathbb{N}} \operatorname{diam}_{H}\left(B_{j}\right)$ and $\varepsilon$ arose in the application of a quasiconformal mapping). The theorem is proved.

Remark. Inequality (23) implies that if $\Omega$ has the interpolation property, then the series $\sum_{j=2}^{\infty} y_{j}^{2}$ is convergent. If the quantities $\operatorname{diam}_{H}\left(B_{j}\right)$ are bounded from above, then $y_{j}$ is comparable with $1-\left|z_{j}\right|$, where $z_{j}$ is the center of the disk $B_{j}$. Hence, if the assumptions of Theorem 3.4 are satisfied, then the series $\sum_{j=1}^{\infty}\left(1-\left|z_{j}\right|\right)^{2}$ converges, moreover, its sum admits estimation in terms of $C_{I}(\Omega), \sup _{j \in \mathbb{N}} \operatorname{diam}_{H} B_{j}$, and the constant $\varepsilon$ of strong separation. This is an analog of the Blaschke condition, we shall discuss this condition in Subsection 6.1 under more general assumptions.

\subsection{Some examples.}

1. Round annulus. Let $B_{1}=\mathcal{B}(0, r), 0<r<1$, and let $\Omega=\mathbb{D} \backslash B_{1}$ be an annulus. It is easy to find a form $\omega \in L_{c}^{2,1}(\Omega)$ with $\operatorname{Per}_{1} \omega=1$ that minimizes $\int_{\Omega}|\omega|^{2} d \lambda_{2}$ over all such forms:

$$
\omega(x+i y)=\frac{x d y-y d x}{2 \pi\left(x^{2}+y^{2}\right)}, \quad x+i y \in \Omega .
$$

Then $\int_{\Omega}|\omega|^{2} d \lambda_{2}=\frac{|\log r|}{2 \pi}$. Hence, $C_{B}^{-1}(\Omega)=C_{I}(\Omega)=\sqrt{\frac{|\log r|}{2 \pi}}$. Thus, if $r \rightarrow 1$, then $C_{B}(\Omega) \rightarrow \infty$. In this case the annulus is too narrow, and then a form with unit period may have small $L^{2}$-norm. If, to the contrary, $r \rightarrow 0$, then $C_{I}(\Omega) \rightarrow \infty$. In this case a 


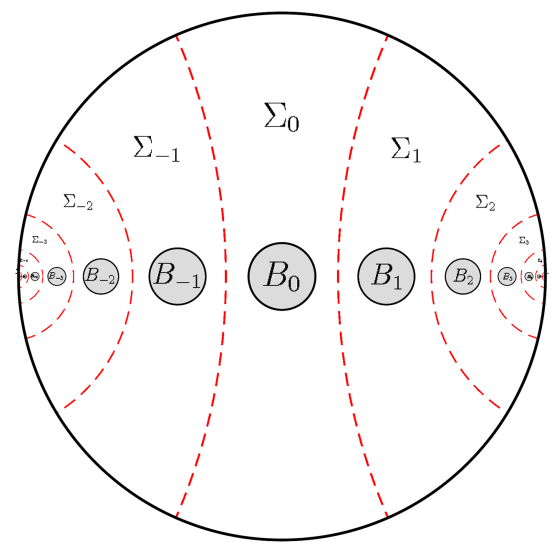

FiguRE 7. A domain with conformal symmetry.

form with unit period is forced to have large $L^{2}$-norm. If $r=0$, then in the degenerate annulus $\mathbb{D} \backslash\{0\}$ there is no closed square-integrable form with nonzero period along a loop winding around the origin. In other words, the $L^{2}$-(co)homologies of the punctured disk are trivial (this property can be understood as the removability of a point). That is why we consider domains with holes consisting of more than one point.

2. A domain with conformal symmetry. Our next example is a domain $\Omega$ having conformal symmetry. Let $a \in(-1,0)$, and let $\varphi(z)=\frac{z-a}{1-a z}$ be a conformal automorphism of the disk $\mathbb{D}$. There exists a domain $\Sigma_{0} \subset \mathbb{D}$ such that the sets $\Sigma_{j}:=\varphi^{j}\left(\Sigma_{0}\right)$ are pairwise disjoint when $j=0, \pm 1, \pm 2, \ldots$, whereas $\mathbb{D} \subset \bigcup_{j=-\infty}^{\infty} \operatorname{clos} \Sigma_{j}$. Such a set $\Sigma_{0}$ is called $a$ fundamental domain of $\varphi$; one can choose $\Sigma_{0}$ bounded by four arcs of circles, symmetric with respect to the origin, and also such that $0 \in \Sigma_{0}$ (see Figure 7).

Take a closed disk $B_{0}$ centered at the origin and lying strictly inside $\Sigma_{0}$. Put $B_{j}=$ $\varphi^{j}\left(B_{0}\right), j= \pm 1, \pm 2, \ldots$, and $\Omega:=\mathbb{D} \backslash \bigcup_{j=-\infty}^{\infty} \varphi^{j}\left(B_{0}\right)$.

All the sets $B_{j}$ are disks obtained from each other by an isometry of the hyperbolic space. Thus, their hyperbolic diameters coincide. It is easy to prove that the sets $B_{j}$ are $\varepsilon$-strongly separated with some $\varepsilon>0$. Thus, by Proposition 2.24 and Theorem 3.2 , the domain $\Omega$ possesses the complete interpolation property.

Estimates for the complete interpolation property of the domain $\Omega$ can be worked out directly. To prove the Bessel property, observe that if $\omega \in L_{c}^{2,1}(\Omega)$, then

$$
\left|\operatorname{Per}_{j} \omega\right|^{2} \leq C \cdot \int_{\Sigma_{j} \backslash B_{j}}|\omega|^{2} d \lambda_{2}
$$

for any $j \in \mathbb{Z}$, with a constant $C<+\infty$ independent of $\omega \in L_{c}^{2,1}(\Omega)$ and of $j$, by the conformal invariance of the last estimate. Summation over all $j \in \mathbb{Z}$ gives the inequality

$$
\sum_{j=-\infty}^{\infty}\left|\operatorname{Per}_{j} \omega\right|^{2} \leq C \cdot \int_{\Omega}|\omega|^{2} d \lambda_{2} .
$$

Thus, $C_{B}(\Omega)<+\infty$.

To prove the interpolation property of $\Omega$ without Theorem 3.2 , let us pick a smooth form $\omega_{0} \in L_{c}^{2,1}\left(\mathbb{D} \backslash B_{0}\right)$ with period 1 around the hole $B_{0}$. For $j=0, \pm 1, \pm 2, \ldots$, we define forms $\omega_{j}=\left(\varphi^{-j}\right)^{\sharp} \omega_{0}$, the pull-backs of $\omega_{0}$ under the action of degrees of $\varphi$. Take 
arbitrary $a=\left\{a_{j}\right\}_{j=-\infty}^{\infty} \in \ell^{2}$. Consider the series

$$
\omega=\sum_{j=-\infty}^{\infty} a_{j} \omega_{j}
$$

Since $\operatorname{Per}_{j} \omega_{k}=\mathbb{1}_{\{j=k\}}$, we have Per $\omega=a$ provided, of course, the series (24) converges in $L_{c}^{2,1}(\Omega)$. To prove this and also to estimate $\|\omega\|_{L_{c}^{2,1}(\Omega)}$ via $\|a\|_{\ell^{2}}$, it suffices to prove that the Gram matrix of the system $\left\{\omega_{j}\right\}_{j=-\infty}^{\infty}$ gives rise to a bounded linear operator in $\ell^{2}$. To this end, observe that $\left\langle\omega_{j}, \omega_{k}\right\rangle_{L_{c}^{2,1}(\Omega)}$ depends only on $|j-k|$ due to the conformal invariance of our construction. Put $I_{j}:=\int_{\Sigma_{j}}\left|\omega_{0}\right|^{2} d \lambda_{2}$. Since the quantities diam $\Sigma_{j}$ decrease exponentially as $|j| \rightarrow \infty$, an estimate of the form $I_{j} \leq C_{1} \cdot \exp \left(-C_{2}|j|\right)$ is fulfilled $\left(C_{1}, C_{2} \in(0,+\infty)\right.$ do not depend on $\left.j\right)$. We have $\left|\left\langle\omega_{0}, \omega_{k}\right\rangle_{L_{c}^{2,1}(\Omega)}\right| \leq \sum_{j=-\infty}^{\infty} \sqrt{I_{j} I_{k-j}}$; dividing the last series into intervals from $-\infty$ to $[k / 2]$ and from $[k / 2]+1$ to $+\infty$ and applying the Cauchy-Schwartz inequality, we derive an estimate of the form $\left|\left\langle\omega_{0}, \omega_{k}\right\rangle_{L_{c}^{2,1}(\Omega)}\right| \leq C_{3} \cdot \exp \left(-C_{4}|k|\right)$ with $C_{3}, C_{4} \in(0,+\infty)$ not depending on $k$. So, $\left|\left\langle\omega_{j}, \omega_{k}\right\rangle_{L_{c}^{2,1}(\Omega)}\right| \leq C_{3} \cdot \exp \left(-C_{4}|j-k|\right)$, and the Young inequality for convolutions shows that the Gram matrix of the system $\left\{\omega_{j}\right\}_{j=-\infty}^{\infty}$ gives a bounded linear operator in $\ell^{2}$.

Note that the complete interpolation property can be proved for other domains having more plentiful group of conformal automorphisms (see also a dyadic example at the end of Subsection 6.1).

3. Inverse domain. The following construction (and also its modification in Example 3.3 below) delivers a counterexample to several naive conjectures on our problem. Also, in this example, only once in this paper, we show formally how to construct a domain with countably many holes from a countable family of domains with finitely many holes by applying to them Möbius automorphisms that make conformal copies of these domain to be far from each other in the hyperbolic metric and, thus, almost independent in the sense of interpolation.

Let us show that there exists a domain $\Omega$ with the complete interpolation property and such that the holes $B_{j}$ in $\Omega$ are disks, but such that the numbers $\operatorname{diam}_{H}\left(B_{j}\right)$ take arbitrarily small values and the sets $B_{j}$ are not strongly separated (Proposition 2.21 and Theorem 3.4 imply that under the complete interpolation condition any of the last two properties implies the other).

Pick a sufficiently small number $\delta>0$. Consider two round holes $B_{1}:=\mathcal{B}\left(0, \frac{1}{2}\right)$, $B_{2}^{\delta}:=\mathcal{B}(1-2 \delta, \delta)$. The holes $B_{1}$ and $B_{2}^{\delta}$ are $\varepsilon$-strongly separated with some $\varepsilon>0$ independent of $\delta$. Moreover, their hyperbolic diameters are bounded from above and separated away from zero uniformly in $\delta$. Put $\Omega_{\delta}=\mathbb{D} \backslash\left(B_{1} \cup B_{2}^{\delta}\right)$ (the left-hand domain in Figure 8). By Proposition 2.24 and Theorem [3.2, the domain $\Omega_{\delta}$ has the complete interpolation property, and moreover, the constants $C_{B}\left(\Omega_{\delta}\right)$ and $C_{I}\left(\Omega_{\delta}\right)$ are bounded from above by some number independent of $\delta$.

Now, let $\widetilde{\Omega}_{\delta}$ be a domain obtained by the inversion $\psi(z)=\frac{1}{2 z}$ applied to $\Omega_{\delta}$. The domain $\widetilde{\Omega}_{\delta}$ is a regular domain in the disk $\mathbb{D}$; we denote by $\widetilde{B}_{1}$ and $\widetilde{B}_{2}^{\delta}$ the holes in $\widetilde{\Omega}_{\delta}$ as in Figure 8 . Let loops $\gamma_{1}$ and $\gamma_{2}$ in $\Omega_{\delta}$ be winding around the holes $B_{1}$ and $B_{2}^{\delta}$, respectively, and put $\widetilde{\gamma}_{1}=\psi\left(\gamma_{1}\right)$ and $\widetilde{\gamma}_{2}=\psi\left(\gamma_{2}\right)$. By Proposition 1.3, the interpolation problem in $\Omega_{\delta}$ for periods along the loops $\gamma_{1}$ and $\gamma_{2}$ is conformally equivalent to the interpolation problem in the domain $\widetilde{\Omega}_{\delta}$ for periods along the loops $\widetilde{\gamma}_{1}$ and $\widetilde{\gamma}_{2}$, that is, the Bessel and interpolation constants in these problems coincide. If $\operatorname{Per}_{1}^{\left(\widetilde{\Omega}_{\delta}\right)}$ and $\operatorname{Per}_{2}^{\left(\widetilde{\Omega}_{\delta}\right)}$ are the functionals of periods of a form of class $L_{c}^{2,1}\left(\widetilde{\Omega}_{\delta}\right)$ around the holes $\widetilde{B}_{1}$ and $\widetilde{B}_{2}$ 


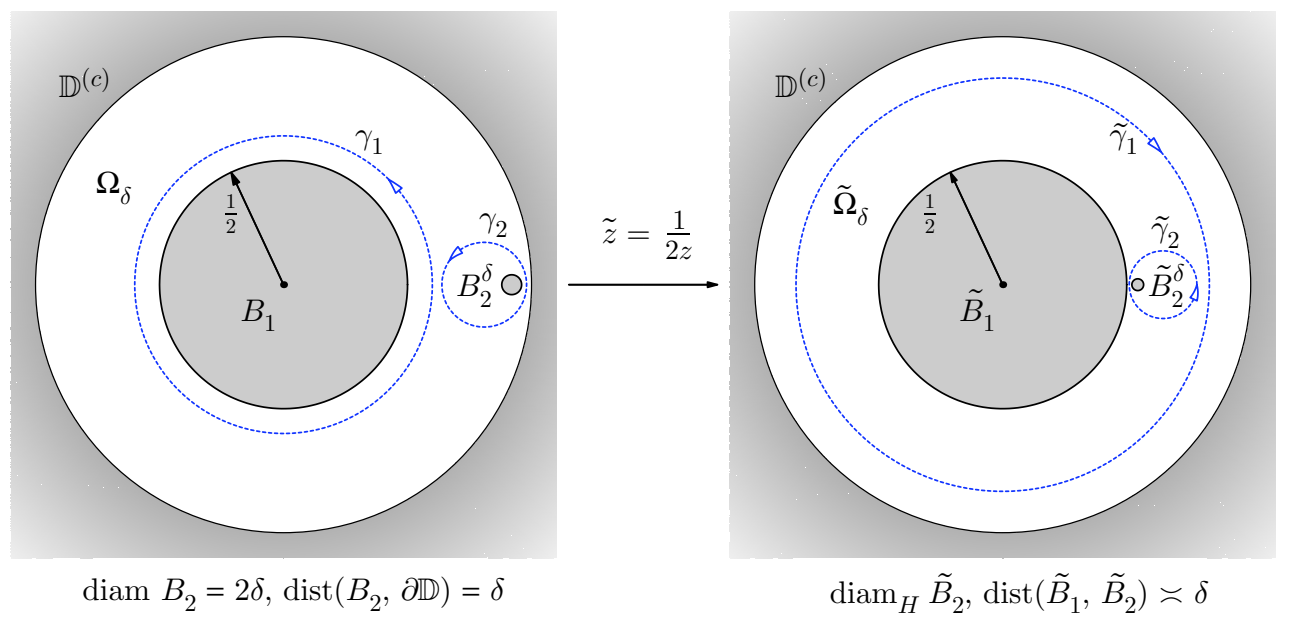

FiguRE 8. Inverse domain.

(respectively), then

$$
\left(\begin{array}{ll}
\operatorname{Per}_{1}^{\left(\widetilde{\Omega}_{\delta}\right)} \widetilde{\omega} \\
\operatorname{Per}_{2}^{\left(\widetilde{\Omega}_{\delta}\right)} \widetilde{\omega}
\end{array}\right)=\left(\begin{array}{cc}
-1 & -1 \\
0 & 1
\end{array}\right)\left(\begin{array}{l}
\int_{\widetilde{\gamma}_{1}} \widetilde{\omega} \\
\int_{\widetilde{\gamma}_{2}} \widetilde{\omega}
\end{array}\right), \quad \widetilde{\omega} \in L_{c}^{2,1}\left(\widetilde{\Omega}_{\delta}\right) .
$$

The $(2 \times 2)$-matrix on the right-hand side of the last relation is bounded and nonsingular. Hence, the Bessel and interpolation constants for the problem in $\widetilde{\Omega}_{\delta}$ and periods around the holes $\widetilde{B}_{1}$ and $\widetilde{B}_{2}$ also stay bounded as $\delta \rightarrow 0$. At the same time, for any fixed $\varepsilon>0$, the holes in $\widetilde{\Omega}_{\delta}$ will not be $\varepsilon$-strongly separated if the number $\delta$ is sufficiently small, and also $\operatorname{diam}_{H} \widetilde{B}_{2}^{\delta \stackrel{\delta \rightarrow 0}{\longrightarrow}} 0$.

Now we show how to construct a domain $\Omega$ possessing the complete interpolation property and such that the hyperbolic diameters of the holes in this domain take arbitrarily small values. Also, the holes in $\Omega$ will not be strongly separated. For this, we apply conformal shifts to the domains $\widetilde{\Omega}_{\delta}$ constructed above. Namely, let $\varphi_{s}(z)=\frac{z-s}{1-z \bar{s}}$ be a Möbius transformation $(s \in \mathbb{D})$. For any sequence $\left\{\delta_{n}\right\}_{n=1}^{\infty} \subset\left(0, \frac{1}{100}\right)$ there exists a real sequence $\left\{s_{n}\right\}_{n=1}^{\infty} \subset(-1,0)$ tending to -1 sufficiently rapidly and such that the sets $\varphi_{s_{n}}\left(\widetilde{B}_{1}^{\delta_{n}} \cup \widetilde{B}_{2}^{\delta_{n}}\right)$ are pairwise disjoint for $n=1,2, \ldots$, while the domain $\Omega=\mathbb{D} \backslash \bigcup_{n=1}^{\infty} \varphi_{s_{n}}\left(\widetilde{B}_{1}^{\delta_{n}} \cup \widetilde{B}_{2}^{\delta_{n}}\right)$ has the complete interpolation property.

First, we show how to ensure the Bessel property of the domain $\Omega$ constructed in such a manner. For $\delta \in\left(0, \frac{1}{100}\right)$ it is easy to show that if $\omega$ is a closed form in the domain $\widetilde{\Omega}_{\delta}$, then

$$
\left\|\operatorname{Per}^{\left(\widetilde{\Omega}_{\delta}\right)} \omega\right\|_{\ell^{2}}^{2} \leq C_{1} \cdot \int_{\widetilde{\Omega}_{\delta} \cap \mathcal{B}(0,3 / 4)}|\omega|^{2} d \lambda_{2}
$$

with some constant $C_{1}<+\infty$ independent of $\delta$. (To prove this, it suffices to enlarge the hole $B_{1}^{\delta}$ in $\Omega_{\delta}$ so that its radius become equal to $\frac{2}{3}$ and apply Proposition 2.24.) By conformal invariance, a similar estimate will be true also for a set of the form $\varphi_{s}\left(\widetilde{\Omega}_{\delta} \cap \mathcal{B}\left(0, \frac{3}{4}\right)\right)$ for periods around the holes $\varphi_{s}\left(\widetilde{B}_{1}^{\delta}\right)$ and $\varphi_{s}\left(\widetilde{B}_{2}^{\delta}\right)$ (for any $s \in \mathbb{D}$ ). Now it suffices to choose numbers $s_{n}, n=1,2, \ldots$, consecutively in order that the sets $\varphi_{s_{n}}\left(\widetilde{\Omega}_{\delta_{n}} \cap \mathcal{B}\left(0, \frac{3}{4}\right)\right)$ be pairwise disjoint, this will immediately lead us to the sequence of periods in the domain $\Omega$. 
Now we show how to ensure the interpolation property of $\Omega$. For any $\delta \in\left(0, \frac{1}{100}\right)$, there exist two forms $w_{1, \delta}, w_{2, \delta} \in L_{c}^{2,1}\left(\widetilde{\Omega}_{\delta}\right)$ for which

$$
\operatorname{Per}^{\left(\widetilde{\Omega}_{\delta}\right)} w_{1, \delta}=(1,0) \text { and } \operatorname{Per}^{\left(\tilde{\Omega}_{\delta}\right)} w_{2, \delta}=(0,1),
$$

whereas $\left\|w_{1, \delta}\right\|_{L_{c}^{2,1}\left(\widetilde{\Omega}_{\delta}\right)} \leq C_{2},\left\|w_{2, \delta}\right\|_{L_{c}^{2,1}\left(\widetilde{\Omega}_{\delta}\right)} \leq C_{2}$ (with $C_{2}<+\infty$ independent of $\delta$ ). This is true because the interpolation constants of the domains $\widetilde{\Omega}_{\delta}$ are bounded uniformly for $\delta<\frac{1}{100}$. Put $w_{1}^{n}:=\left(\varphi_{s_{n}}^{-1}\right)^{\sharp} w_{1, \delta_{n}}, w_{2}^{n}:=\left(\varphi_{s_{n}}^{-1}\right)^{\sharp} w_{2, \delta_{n}}$. Then $w_{1}^{n}, w_{2}^{n} \in L_{c}^{2,1}(\Omega)$. If $a=\left(a_{1}^{1}, a_{2}^{1}, a_{1}^{2}, a_{2}^{2}, \ldots\right) \in \ell^{2}$ is a real sequence and

$$
\omega=\sum_{j=1}^{\infty}\left(a_{1}^{j} w_{1}^{j}+a_{2}^{j} w_{2}^{j}\right)
$$

then $\operatorname{Per}^{(\Omega)} \omega=a$. To estimate $\|\omega\|_{L_{c}^{2,1}(\Omega)}$ in terms of $\|a\|_{\ell^{2}}$ (and to prove the $L^{2}$-convergence of the series defining this form), we must check that the Gram matrix of the system $\left\{w_{1}^{n}, w_{2}^{n}\right\}_{n=1}^{\infty}$ determines a bounded linear operator from $\ell^{2}$ into $\ell^{2}$ (see [2]). Let us choose numbers $s_{n}$ consecutively for $n=1,2, \ldots$ If numbers $s_{1}, \ldots, s_{n-1}$ are chosen and $s_{n} \rightarrow-1$, then for $\alpha, \beta=1,2$ and $m=1, \ldots, n-1$ it is easy to prove that

$$
\int_{\varphi_{s_{m}}\left(\widetilde{\Omega}^{\delta_{m}}\right) \cap \varphi_{s_{n}}\left(\widetilde{\Omega}^{\delta_{n}}\right)}\left|w_{\alpha}^{m}\right| \cdot\left|w_{\beta}^{n}\right| d \lambda_{2} \rightarrow 0 .
$$

Hence, the numbers $s_{n}$ can be chosen so that the scalar products $\left\langle w_{\alpha}^{n}, w_{\beta}^{m}\right\rangle_{L_{c}^{2,1}(\Omega)}(m \neq n$, $\alpha, \beta=1,2)$ be arbitrarily small in any norm. In particular, under an appropriate choice of the sequence $\left\{s_{n}\right\}_{n=1}^{\infty}$, the Gram matrix of the system $\left\{w_{1}^{n}, w_{2}^{n}\right\}_{n=1}^{\infty}$ can be made arbitrarily close in the Hilbert-Schmidt norm to the matrix formed by the blocks

$$
\left(\begin{array}{cc}
\left\langle w_{1}^{n}, w_{1}^{n}\right\rangle_{L_{c}^{2,1}(\Omega)} & \left\langle w_{1}^{n}, w_{2}^{n}\right\rangle_{L_{c}^{2,1}(\Omega)} \\
\left\langle w_{2}^{n}, w_{1}^{n}\right\rangle_{L_{c}^{2,1}(\Omega)} & \left\langle w_{2}^{n}, w_{2}^{n}\right\rangle_{L_{c}^{2,1}(\Omega)}
\end{array}\right) .
$$

But such a matrix determines a bounded operator due to estimates on $\left\|w_{1, \delta}\right\|_{L_{c}^{2,1}\left(\widetilde{\Omega}_{\delta}\right)}$ and $\left\|w_{2, \delta}\right\|_{L_{c}^{2,1}\left(\tilde{\Omega}_{\delta}\right)}$; this implies the boundedness of the Gram matrix of the system $\left\{w_{1}^{n}, w_{2}^{n}\right\}_{n=1}^{\infty}$. Thus, under an appropriate choice of the numbers $s_{n}, n=1,2, \ldots$, the domain $\Omega$ will have the interpolation property.

It is easily seen that if $\delta_{n} \stackrel{n \rightarrow \infty}{\longrightarrow} 0$, then $\operatorname{diam}_{H} \varphi_{s_{n}}\left(\widetilde{B}_{2}^{\delta_{n}}\right) \stackrel{n \rightarrow \infty}{\longrightarrow} 0$ and

$$
\frac{\operatorname{dist}\left(\varphi_{s_{n}}\left(\widetilde{B}_{1}^{\delta_{n}}\right), \varphi_{s_{n}}\left(\widetilde{B}_{2}^{\delta_{n}}\right)\right)}{\operatorname{diam} \varphi_{s_{n}}\left(\widetilde{B}_{1}^{\delta_{n}}\right)} \stackrel{n \rightarrow \infty}{\longrightarrow} 0 .
$$

Thus, there exist regular domains $\Omega$ with round holes that possess the complete interpolation property and are such that the holes in $\Omega$ are not strongly separated, and also the hyperbolic diameters of these holes take arbitrarily small values.

Yet another property of the resulting domain $\Omega$ should be mentioned. Recall that if $\Omega^{\prime}$ is some regular domain and $B_{1}, B_{2}, \ldots$ are holes in $\Omega^{\prime}$, then $\mu_{\Omega^{\prime}}=\sum_{j=1}^{\infty} \frac{\mathbb{1}_{B_{j} \cdot \lambda_{2}}}{\lambda_{2}\left(B_{j}\right)}$ is the measure associated with this domain. Notice that the contribution of the hole $\widetilde{B}_{2}^{\delta}$ to $\mu_{\widetilde{\Omega}_{\delta}}$ is weakly* close to a unit point mass if $\delta \rightarrow 0$. Thus, it is easy to prove that the norm of the embedding operator of $\stackrel{\circ}{W}^{1,2}(\mathbb{D})$ into $L^{2}\left(\mu_{\widetilde{\Omega}_{\delta}}\right)$ tends to $+\infty$ as $\delta \rightarrow 0$; at the same time, a large norm in $L^{2}\left(\mu_{\widetilde{\Omega}_{\delta}}\right)$ can be given by functions constant on $\widetilde{B}_{2}^{\delta}$ and having unit norms in $\stackrel{\circ}{W}^{1,2}(\mathbb{D})$. Due to the conformal invariance of the Dirichlet integral, the norms of embeddings of $\stackrel{\circ}{W}^{1,2}(\mathbb{D})$ into $L^{2}\left(\frac{\mathbb{1}_{B} \cdot \lambda_{2}}{\lambda_{2}(B)}\right)$, where $B=\varphi_{s_{n}}\left(\widetilde{B}_{2}^{\delta_{n}}\right)$, will also tend to infinity as $\delta_{n} \rightarrow 0$ (for any choice of the numbers $s_{n}$ ). But this implies that the embedding operator from $\stackrel{\circ}{W}^{1,2}(\mathbb{D})$ into $L^{2}\left(\mu_{\Omega}\right)$ will not be bounded. Thus, the complete interpolation property of a domain $\Omega$ does not, in general, imply the (MC) property 
of the measure $\mu_{\Omega}$ (though the estimate $\|u\|_{L^{2}\left(\mu_{\Omega}\right)} \leq C_{I}(\Omega) \cdot\|u\|_{W^{1,2}(\mathbb{D})}$ for functions constant on any hole in $\Omega$ is equivalent to the interpolation property of such a domain). 4. Composition of inversions. The example of an inverse domain constructed above leads us to the following natural question: what will happen if we make a larger number of inversions? Given a number $M \in \mathbb{N}$ and numbers $s_{1}, s_{2}, \ldots, s_{M} \in(-1,0)$ close to -1 , let us construct a domain $\Omega_{M}$ with a step-by-step process. At the $m$ th step $(m=1,2, \ldots, M)$, we shall construct a domain $\Omega_{m}$ whose holes are disks $B_{1, m}$, $B_{2, m}, \ldots, B_{m, m} \subset \mathbb{D}$; also, we shall construct closed oriented curves $\beta_{1, m}, \beta_{1, m}, \ldots, \beta_{m, m}$ in $\Omega_{m}$.

Put $B_{1,1}=\overline{\mathcal{B}}\left(0, \frac{1}{2}\right), \Omega_{1}=\mathbb{D} \backslash B_{1,1}$ and let $\beta_{1,1}$ be a circle placed in $\Omega_{1}$ and winding around $B_{1,1}$ counterclockwise. As in the previous example, we put $\psi(z)=\frac{1}{2 z}, \varphi_{s}(z)=$ $\frac{z-s}{1-\bar{s} z}, s, z \in \mathbb{D}$. Suppose that $m=1,2, \ldots, M-1$ and that disks $B_{1, m}, B_{2, m}, \ldots, B_{m, m}$ are already constructed. For $s_{m}$ sufficiently close to -1 , put $B_{j, m+1}^{\prime}:=\varphi_{s_{m}}\left(B_{j, m}\right), \beta_{j, m+1}^{\prime}:=$ $\varphi_{s_{m}}\left(\beta_{j, m}\right)$ for $j=1,2, \ldots, m$, and $B_{m+1, m+1}^{\prime}:=\overline{\mathcal{B}}\left(0, \frac{1}{2}\right), \Omega_{m+1}^{\prime}:=\mathbb{D} \backslash \bigcup_{j=1}^{m+1} B_{j, m+1}^{\prime}$; the curve $\beta_{m+1, m+1}^{\prime}$ is defined as a loop in $\Omega_{m+1}^{\prime}$ winding around $B_{m+1, m+1}^{\prime}$ counterclockwise and not winding around other holes in $\Omega_{m+1}^{\prime}$. Now we put $B_{j, m+1}:=\psi\left(B_{j, m+1}^{\prime}\right)$, $\beta_{j, m+1}:=\psi\left(\beta_{j, m+1}^{\prime}\right)$ for $j=1,2, \ldots, m, \beta_{m+1, m+1}:=\psi\left(\beta_{m+1, m+1}^{\prime}\right), B_{m+1, m+1}:=$ $\overline{\mathcal{B}}\left(0, \frac{1}{2}\right)$, and $\Omega_{m+1}:=\mathbb{D} \backslash \bigcup_{j=1}^{m+1} B_{j, m+1}$.

Performing this construction consecutively for $m=1,2, \ldots, M-1$, we obtain domains $\Omega_{m}, \Omega_{m}^{\prime}, m=1,2, \ldots, M$. For such $m$, we state the problem on interpolation by periods in $\Omega_{m}$ along the curves $\beta_{1}, \beta_{2}, \ldots, \beta_{m}$, and also this problem in $\Omega_{m}^{\prime}$ along the curves $\beta_{1}^{\prime}, \beta_{2}^{\prime}, \ldots, \beta_{m}^{\prime}$ (see the end of Subsection 1.1).

Take a number $C>\max \left\{C_{B}\left(\Omega_{1} ; \beta_{1}\right), C_{I}\left(\Omega_{1} ; \beta_{1}\right)\right\}$. Using induction on $m$, we prove that, under an appropriate choice of numbers $s_{m}$, the inequality

$$
\max \left\{C_{B}\left(\Omega_{m} ; \beta_{1}, \beta_{2}, \ldots, \beta_{m}\right), C_{I}\left(\Omega_{1} ; \beta_{1}, \beta_{2}, \ldots, \beta_{m}\right)\right\}<C
$$

is fulfilled for all $m=1,2, \ldots, M$. Indeed, if this inequality is valid for the domain $\Omega_{m}$, then, using the conformal invariance of the problem and arguing like in Example 3.3. it is easy to show that

$$
\max \left\{C_{B}\left(\Omega_{m+1}^{\prime} ; \beta_{1}^{\prime}, \beta_{2}^{\prime}, \ldots, \beta_{m}^{\prime}, \beta_{m+1}^{\prime}\right), C_{I}\left(\Omega_{m+1}^{\prime} ; \beta_{1}^{\prime}, \beta_{2}^{\prime}, \ldots, \beta_{m}^{\prime}, \beta_{m+1}^{\prime}\right)\right\}<C
$$

if $s_{m}$ is sufficiently close to -1 . But then

$$
\max \left\{C_{B}\left(\Omega_{m+1} ; \beta_{1}, \beta_{2}, \ldots, \beta_{m}, \beta_{m+1}\right), C_{I}\left(\Omega_{m+1} ; \beta_{1}, \beta_{2}, \ldots, \beta_{m}, \beta_{m+1}\right)\right\}<C,
$$

again due to conformal invariance.

Let $j=1,2, \ldots, M$, and let $\gamma_{j}$ be a loop winding around the hole $B_{j, M}$ in $\Omega_{M}$ in the positive direction and not winding around the other holes in this domain. It easily seen that in the space $H_{1, c}\left(\Omega_{M}\right)$ of compact homologies we have $\beta_{1}=\gamma_{1}, \beta_{j}=-\sum_{k=1}^{j} \gamma_{k}$ for $j=2,3, \ldots, M$. (For example, the second relation should be understood as follows: $\int_{\beta_{j}} \omega=-\sum_{k=1}^{j} \int_{\gamma_{k}} \omega$ for any $\omega \in L_{c}^{2,1}\left(\Omega_{M}\right)$.) Let $A$ be the $(M \times M)$-matrix that maps the period vector of a form $\omega \in L_{c}^{2,1}\left(\Omega_{M}\right)$ along the curves $\gamma_{1}, \gamma_{2}, \ldots, \gamma_{M}$ to the vector of $\omega$ 's periods along the loops $\beta_{1}, \beta_{2}, \ldots, \beta_{M}$ (cf. (25)). Then the norm of the matrix $A$ with respect to the Euclidean norm in $\mathbb{R}^{M}$ is greater than $\sqrt{M}$. Since $C_{B}\left(\Omega_{m+1} ; \beta_{1}, \beta_{2}, \ldots, \beta_{M}\right)<C$, this implies that $C_{I}\left(\Omega_{m+1} ; \gamma_{1}, \gamma_{2}, \ldots, \gamma_{M}\right) \geq \sqrt{M} / C$.

So, if in $\Omega_{M}$ we state the interpolation problem for periods around holes in this domain as we usually do, then the interpolation constant for such a problem will be large if $M$ is large; one can prove the same about the Bessel constant for this problem. This naturally leads us to the following question: do there exist domains $\Omega$ such that the constants $C_{B}(\Omega)$ and $C_{I}(\Omega)$ are not very large, but the disk $\mathcal{B}(0,2 / 3)$ intersects a very large number of holes in $\Omega$ ? A negative answer will be given in 4 . 
The example constructed above also leads us to the statement of the problem on integral Riesz bases in Hilbert homologies, which will be discussed in Subsection 6.3 .

3.4. Partial criterion. We summarize the results of Proposition 2.24 , Theorem 3.2 , Proposition 2.21, Theorem 3.4, and Example 3.3 in Subsection 3.3 in the following theorem.

Theorem 3.7. Let $\Omega$ be a regular domain with round holes $B_{j}, j=1,2, \ldots$ Suppose also that $\sup _{j \in \mathbb{N}} \operatorname{diam}_{H}\left(B_{j}\right)<+\infty$.

(1) Suppose that the holes $B_{j}$ are $\varepsilon$-strongly separated with some $\varepsilon>0$ and that $\inf _{j \in \mathbb{N}} \operatorname{diam}_{H}\left(B_{j}\right)>0$. Then $\Omega$ posseses the complete interpolation property. Moreover, the constants $C_{B}(\Omega)$ and $C_{I}(\Omega)$ can be estimated from above only in terms of $\sup _{j \in \mathbb{N}} \operatorname{diam}_{H}\left(B_{j}\right), \inf _{j \in \mathbb{N}} \operatorname{diam}_{H}\left(B_{j}\right)$, and $\varepsilon$.

(2) Suppose that $\inf _{j \in \mathbb{N}} \operatorname{diam}_{H}\left(B_{j}\right)>0$ and $\Omega$ possesses the complete interpolation property. Then the holes $B_{j}$ are $\varepsilon$-strongly separated with some $\varepsilon>0$ depending only on $\inf _{j \in \mathbb{N}} \operatorname{diam}_{H}\left(B_{j}\right)$ and $C_{B}(\Omega)$.

If, vice versa, the holes $B_{j}$ are $\varepsilon$-strongly separated for some $\varepsilon>0$ and $\Omega$ has the complete interpolation property, then $\inf _{j \in \mathbb{N}} \operatorname{diam}_{H}\left(B_{j}\right)$ is strictly positive and can be estimated from below only in terms of $\sup _{j \in \mathbb{N}} \operatorname{diam}_{H}\left(B_{j}\right), \varepsilon$, and $C_{I}(\Omega)$.

(3) There exist regular domains $\Omega$ of the form $\mathbb{D} \backslash \bigcup_{j=1}^{\infty} B_{j}$, where each $B_{j}, j=$ $1,2, \ldots$, is a disk, that have the complete interpolation property and are such that $\inf _{j \in \mathbb{N}} \operatorname{diam}_{H}\left(B_{j}\right)=0$ and the holes $B_{j}$ are not $\varepsilon$-strongly separated for any $\varepsilon>0$.

The first two assertions of this theorem give a criterion for complete interpolation for domains with round holes in the case where one of the two following conditions is satisfied: either $\inf _{j \in \mathbb{N}} \operatorname{diam}_{H}\left(B_{j}\right)>0$, or the holes $B_{j}$ are strongly separated. But none of these two condition is necessary for complete interpolation - this is the third assertion of the theorem.

In fact, first two assertions of Theorem 3.7 are true for domains with holes of arbitrary form, not necessary round. Propositions 2.24 and 2.21 were proved without the assumption that all the sets $B_{j}$ are disks. The sufficiency of the hypothesis of the first assertion in Theorem 3.7 for the interpolation property was proved in Theorem 3.3 . The validity of Theorem 3.4 on the lower estimate for hyperbolic diameters also stays true when the $B_{j}$ are not necessarily disks. This can be derived from Theorem 5.4 (see below), taking into account Proposition 2.24 and Theorem 4.2 on uniform local finiteness, which we turn to right now.

\section{§4. UNIFORM LOCAL FINITENESS OF THE FAMILY OF HOLES}

Theorem 3.7 easily implies that if a domain $\Omega$ with round holes has the complete interpolation property and if either $\inf _{j \in \mathbb{N}} \operatorname{diam}_{H}\left(B_{j}\right)>0$ or the holes $B_{j}$ are $\varepsilon$-strongly separated, then for any $R<1$ the number of holes $B_{j}$ intersecting $\mathcal{B}(0, R)$ can be estimated from above in terms of $C_{B}(\Omega), C_{I}(\Omega), R$, and either $\varepsilon$ or $\inf _{j \in \mathbb{N}} \operatorname{diam}_{H}\left(B_{j}\right)$, respectively; due to conformal invariance, the same estimate will be true if we replace the disk $\mathcal{B}(0, R)$ by any conformal copy of it obtained by a Möbius transformation. Our goal is to prove the same estimate for arbitrary complete interpolation domains without any assumptions on strong separation or on the inequality $\inf _{j \in \mathbb{N}} \operatorname{diam}_{H}\left(B_{j}\right)>0$.

Definition 4.1. We say that the family of holes in a domain $\Omega$ is uniformly locally finite if there exists $N \in \mathbb{N}$ such that any disk in the hyperbolic metric having radius 1 in this metric intersects at most $N$ sets $B_{j}$. The smallest $N$ with this property is called the constant of uniform local finiteness of the domain $\Omega$ and is denoted by $N(\Omega)$. 
Let $d>0$. It is easily seen that if the uniform local finiteness property is satisfied, then any set of hyperbolic diameter not exceeding $d$ intersects at most $\tilde{N}$ sets $B_{j}$, where $\tilde{N}<+\infty$ can be estimated from above in terms of $d$ and $N(\Omega)$. We denote this number by $N(\Omega, d)$, having in mind that it only depends on $d$ and $N(\Omega)$.

Theorem 4.2. If a regular domain $\Omega$ possesses the interpolation and weak Bessel properties, then it possesses the uniform local finiteness property. Moreover, the constant $N(\Omega)$ can be estimated from above via $C_{I}(\Omega)$ and $\widetilde{C}_{B}(\Omega)$ only.

If $\widetilde{C}_{B}(\Omega)<+\infty$, then $C_{B}(\Omega)<+\infty$ (Theorem 2.16). In the argument below, nevertheless, we shall use only the estimate $\widetilde{C}_{B}(\Omega)<+\infty$.

Proof of Theorem 4.2. Due to the conformal invariance of the problem, it suffices to estimate the number of holes intersecting $\mathcal{B}\left(0, \frac{1}{2}\right)$.

First, we employ the interpolation property of $\Omega$. We are going to construct a function $u \in \mathcal{A d m}(\Omega)$ so that the values $\left.u\right|_{B_{j}}$ admit a lower estimate, while the norm $\|u\|_{W^{1,2}(\mathbb{D})}$ is not too large. Then the estimate in the second assertion of Proposition 2.10 will lead us to the desired claim.

For this, we take a risk: let us look for a function $u \in \mathcal{A d m}(\Omega)$ such that $|\nabla u| \leq 1$ almost everywhere. Put $a_{j}=\left.u\right|_{B_{j}}, j=1,2, \ldots$ Investigation of the extremal problem

$$
\left\{\begin{array}{l}
\left|a_{j}-a_{k}\right| \leq \operatorname{dist}\left(B_{j}, B_{k}\right), j, k \in \mathbb{N} ; \quad\left|a_{j}\right| \leq \operatorname{dist}\left(B_{j}, \partial \mathbb{D}\right), j \in \mathbb{N} ; \\
\sum_{j=1}^{\infty} a_{j}^{2} \longrightarrow \max
\end{array}\right.
$$

with countable number of real unknowns $\left\{a_{j}\right\}_{j=1}^{\infty}$ leads us to the idea of application of a special inner metric in $\mathbb{C}$. We have already used this idea in the proof of Proposition 2.10.

Now we pass to a formal argument. Everywhere in this proof we consider polygonal chains in $\mathbb{C}$ with finite number of vertices, i.e., piecewise-linear continuous mappings $\Gamma:[0, T] \rightarrow \mathbb{C}, T<+\infty$. We also assume that such a polygonal chain $\Gamma$ is endowed with an orientation and is parametrized naturally, that is $\left|\Gamma^{\prime}(t)\right|=1$ on $[0, T]$ almost everywhere with respect to the one-dimensional Lebesgue measure $\lambda_{1}$. If the mapping $\Gamma$ is injective, then we say that such a chain is simple. If $\Gamma(0)=z_{1}, \Gamma(T)=z_{2}$, then we say that the chain $\Gamma$ joins $z_{1}$ and $z_{2}$.

In $\mathbb{C}$, consider a degenerate metric $\mathbb{1}_{\Omega}|d z|$. For a polygonal chain $\Gamma$ in $\mathbb{C}$ we put

$$
L(\Gamma):=\lambda_{1}\left(\Gamma^{-1}(\Omega)\right) .
$$

(The set $\Gamma^{-1}(\Omega)$ is open in $\mathbb{R}$ and, thus, is Lebesgue measurable.) If $\Gamma:[0, T] \rightarrow \mathbb{C}$ is a simple polygonal chain in $\mathbb{C}$, then $L(\Gamma)=\mathcal{H}^{1}(\Gamma[0, T] \cap \Omega)$, where the symbol $\mathcal{H}^{1}$ stands for the one-dimensional Hausdorff measure, i.e., length. If $\Gamma[0, T] \subset \Omega$ and a polygonal chain $\Gamma$ is simple, then $L(\Gamma)=\mathcal{H}^{1}(\Gamma[0, T])$; if, to the contrary, $\Gamma[0, T] \subset \mathbb{D}^{(c)}$ or $\Gamma[0, T] \subset B_{j}$ for some $j=1,2, \ldots$, then $L(\Gamma)=0$.

We introduce the following degenerate metric:

$$
\rho\left(z_{1}, z_{2}\right):=\inf \left\{L(\Gamma): \Gamma \text { is a polygonal chain joining } z_{1} \text { and } z_{2}\right\}, \quad z_{1}, z_{2} \in \mathbb{C} .
$$

As such a chain $\Gamma$, we may take the line segment $\left[z_{1}, z_{2}\right]$ joining $z_{1}$ and $z_{2}$ and endowed with natural parametrization; therefore,

$$
\rho\left(z_{1}, z_{2}\right) \leq L\left(\left[z_{1}, z_{2}\right]\right) \leq\left|z_{1}-z_{2}\right| .
$$

Recall that any set $B_{j}$ is a closure of a domain with sufficiently smooth boundary. Thus, any two points $z_{1}, z_{2} \in B_{j}$ can be joined by a polygonal chain $\Gamma$ lying in $B_{j}$; for such a chain we have $L(\Gamma)=0$. Thus, $\rho\left(z_{1}, z_{2}\right)=0$. So, all the holes $B_{j}$ collapse into points in the degenerate metric $\rho$. 
Note that any line segment lying in $\Omega$ will be geodesic (that is a locally shortest curve) in the metric $\rho$. Also, all curves lying in some hole or in $\mathbb{D}^{(c)}$ will also be geodesics.

For $z \in \mathbb{C}$, put

$$
\begin{aligned}
u(z) & :=\operatorname{dist}_{\rho}\left(z, \mathbb{D}^{(c)}\right) \\
& =\inf \left\{L(\Gamma): \Gamma \subset \mathbb{C} \text { is a polygonal chain joining } z \text { with a point in } \mathbb{D}^{(c)}\right\} .
\end{aligned}
$$

From (26) we see that

$$
u\left(z_{2}\right) \leq u\left(z_{1}\right)+\left|z_{1}-z_{2}\right|
$$

for any $z_{1}, z_{2} \in \mathbb{C}$. Hence, the function $u$ is Lipschitz and $|\nabla u| \leq 1$ almost everywhere in $\mathbb{C}$. By definition, $u=0$ on $\partial \mathbb{D}$. Thus, $u \in \stackrel{\circ}{W}^{1,2}(\mathbb{D})$. Next, $\rho\left(z_{1}, z_{2}\right)=0$ if $z_{1}, z_{2} \in B_{j}$ for some $j$, and then $u$ is constant on each hole $B_{j}$. So, $u \in \mathcal{A} d m(\Omega)$ and $\|u\|_{W^{1,2}(\mathbb{D})} \leq \sqrt{\pi}$.

Now, relying only on the weak Bessel property, we are going to prove that the values $\left.u\right|_{B_{j}}$ cannot be very small.

Lemma 4.3. Suppose that $\Omega$ is a domain with weak Bessel property, i.e.,

$$
\operatorname{Cap}_{2}\left(B_{j}, \Omega^{(c)} \backslash B_{j}\right) \leq \widetilde{C}_{B}^{2}(\Omega)<+\infty
$$

for any $j=1,2, \ldots$ (see Proposition 2.15). Then, for any $j=1,2, \ldots$, the function $u$ defined above satisfies the estimate

$$
\left.u\right|_{B_{j}} \geq \frac{\exp \left(-2 \pi \widetilde{C}_{B}^{2}(\Omega)\right)}{2} \cdot \operatorname{dist}\left(B_{j}, \partial \mathbb{D}\right) .
$$

Proof. Let $\Gamma:[0, T] \rightarrow \mathbb{C}, T<+\infty$, be a polygonal chain joining a point in $B_{j}$ with $\partial \mathbb{D}$. We must prove that

$$
\lambda_{1}\left(\Gamma^{-1}(\Omega)\right) \geq \frac{\exp \left(-2 \pi \widetilde{C}_{B}^{2}(\Omega)\right)}{2} \cdot \operatorname{dist}\left(B_{j}, \partial \mathbb{D}\right) .
$$

Let us make some technical assumptions.

We may prove the desired estimate assuming that the number of holes in $\Omega$ is finite. Indeed, supposing that $j=1$, let us erase the holes $B_{\nu+1}, B_{\nu+2}, \ldots$ and put $\Omega_{\nu}:=$ $\mathbb{D} \backslash \bigcup_{j^{\prime}=1}^{\nu} B_{j^{\prime}}$, here $\nu=1,2, \ldots$. For any such $\nu$ and any $j^{\prime}=1,2, \ldots, \nu$, the estimate $\mathrm{Cap}_{2}\left(B_{j^{\prime}}, \Omega_{\nu}^{(c)} \backslash B_{j^{\prime}}\right) \leq \widetilde{C}_{B}^{2}(\Omega)$ stays true. If we prove inequalities (28) for the domain $\Omega=\Omega_{\nu}$ and for all $\nu$, then this inequality for the initial domain $\Omega$ will be obtained by passing to the limit as $\nu \rightarrow \infty$ because $\lambda_{1}\left(\Gamma^{-1}\left(\Omega_{1}\right)\right) \leq T<+\infty$.

We may assume that $\Gamma \cap B_{j^{\prime}}$ is connected for any $j^{\prime}=1,2, \ldots$ Indeed, consider the first (with respect to motion along $\Gamma$ ) set $B_{j^{\prime}}$ that $\Gamma$ intersects twice; the arc of the curve $\Gamma$ from its first entrance into $B_{j^{\prime}}$ until its last exit from $B_{j^{\prime}}$ can be replaced by a naturally parametrized polygonal chain in $B_{j^{\prime}}$ (recall that we assume that the boundary $\partial B_{j^{\prime}}$ is smooth). Such a replacement does not increase $L(\Gamma)$. Next, if the resulting curve intersects some hole twice, then we perform the same procedure with this curve, and so on. The number of holes in $\Omega$ is finite by the assumption made above; then we shall only make finitely many changes with our curve $\Gamma$, and hence, it will remain a polygonal chain with finitely many vertices.

Next, if $\left(t_{1}, t_{2}\right)$ is a maximal interval in $[0, T]$ such that $\Gamma\left(\left(t_{1}, t_{2}\right)\right) \subset \Omega$, then we replace the arc $\left.\Gamma\right|_{\left(t_{1}, t_{2}\right)}$ in our curve by the line segment $\left[\Gamma\left(t_{1}\right), \Gamma\left(t_{2}\right)\right]$. Such a replacement does not increase the length $L(\Gamma)$. Make such replacements for all connected components of $\Gamma^{-1}(\Omega) \subset[0, T]$. If necessary, reparametrize the resulting curve naturally. If the new curve intersects some hole $B_{j^{\prime}}$ by a nonconnected set, then, again, we replace the arc of the curve from its first entrance into $B_{j^{\prime}}$ until its last exit from $B_{j^{\prime}}$ by a polygonal 


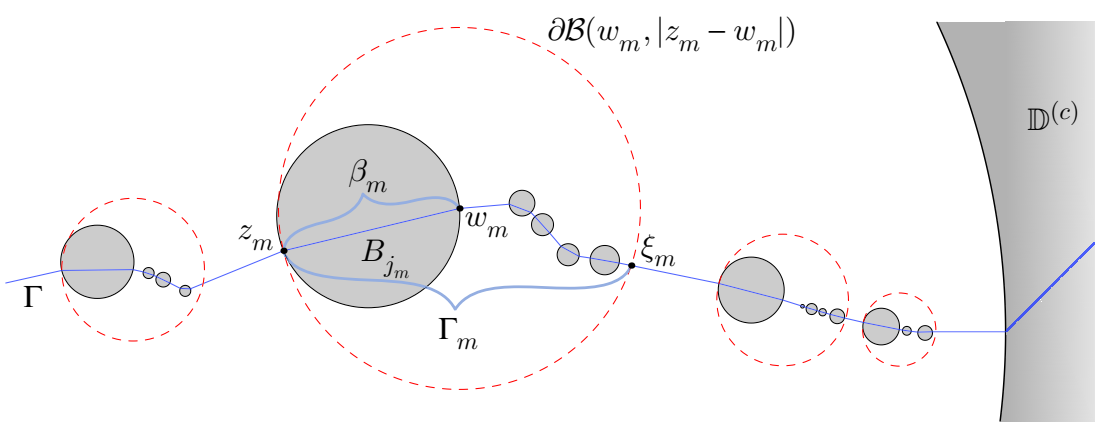

FiguRE 9. Subdivision of the curve $\Gamma$.

chain lying in $B_{j^{\prime}}$. Thus, we may assume that $\Gamma([0, T]) \cap \Omega$ consists of finitely many line segments, whereas all the sets $\Gamma \cap B_{j^{\prime}}$ are connected, $j^{\prime}=1,2, \ldots$

If the polygonal chain $\Gamma$ intersects some hole $B_{j^{\prime}}$ by at least two points, then we assume that all the interior points of the $\operatorname{arc} \Gamma \cap B_{j^{\prime}}$ lie in $\operatorname{Int} B_{j^{\prime}}$. This can be achieved by an appropriate correction of our chain.

We may also assume that $\Gamma$ is a simple polygonal chain, because self-intersections increase $L(\Gamma)$.

Finally, we make one more assumption. The polygonal chain $\Gamma$ ends at a point $\zeta \in \partial \mathbb{D}$. Attach to $\Gamma$ a line segment of length 5 starting at $\zeta$ in order that the end of the curve $\Gamma$ be placed at the distance 6 from the origin. On the other hand, we may assume that $\Gamma$ starts at a point $w \in \partial B_{j}$. Choose $z \in \operatorname{Int} B_{j}, z \neq w$, such that the line segment $[z, w)$ lies in Int $B_{j}$ entirely, and attach this segment to $\Gamma$. Now we assume that $\Gamma$ starts at a point $z \in \operatorname{Int} B_{j}$.

Our goal in the argument below is as follows: we construct a covering of the set $\Gamma \cap \Omega^{(c)}$ by $\operatorname{arcs} \Gamma_{m}(m=0,1, \ldots)$ of $\Gamma$ in such a way that in any of the $\operatorname{arcs} \Gamma_{m}$ one of the arcs $B_{j^{\prime}} \cap \Gamma, j^{\prime}=1,2, \ldots$, will have a significant weight (see Figure 9). This arc will be denoted by $\beta_{m}$, its beginning (according to the motion along $\Gamma$ ) by $z_{m}$, and its end by $w_{m}$. Then the weight of the set $\left(\Gamma_{m} \cap \Omega^{(c)}\right) \backslash \beta_{m}$ on the arc $\Gamma_{m}$ will not be large (from capacity considerations), while the set $\Gamma_{m} \cap \Omega$ will, on the contrary, be large. This will lead us to the desired estimate. The construction will be worked out by a step-by-step process.

Lemma 4.4. Under the conditions of Lemma 4.3 and the assumptions made at the beginning of its proof, there exists a number $M \in \mathbb{N}$, a sequence of points $z_{0}, w_{0}, \xi_{0}, z_{1}$, $w_{1}, \xi_{1}, \ldots, z_{M}, w_{M}, \xi_{M}$ on the curve $\Gamma$, closed arcs $\Gamma_{0}, \beta_{0}, \Gamma_{1}, \beta_{1}, \ldots, \Gamma_{M}, \beta_{M}$ on this curve, and a sequence of distinct indices $j_{0}, j_{1}, \ldots, j_{M} \in \mathbb{N}$ such that:

(1) the sequence of points $z_{0}, w_{0}, \xi_{0}, z_{1}, w_{1}, \xi_{1} \ldots, z_{M}, w_{M}, \xi_{M}$ is ordered in the direction of motion along the oriented curve $\Gamma$ (that is, in the order of increase of its parameter);

(2) for $m=0,1, \ldots, M$, the arc $\beta_{m}$ is the arc of the polygonal chain $\Gamma$ starting at $z_{m}$ and ending at $w_{m}$; any such arc $\beta_{m}$ lies entirely in the hole $B_{j_{m}}$; the point $w_{m}$ is the point of exit of $\Gamma$ from $B_{j_{m}}$;

(3) for $m=0,1, \ldots, M$, the arc $\Gamma_{m}$ starts at $z_{m}$ and ends at $\xi_{m}$; moreover, we have $\left|\xi_{m}-w_{m}\right|=\left|z_{m}-w_{m}\right|$; the arc $\Gamma_{m} \backslash \beta_{m}$ does not intersect $B_{j_{m}}$ (but may intersect other holes);

(4) distinct arcs $\Gamma_{m}, m=0,1, \ldots, M$, do not intersect one another at inner points; $\Gamma \cap(\mathbb{D} \backslash \Omega) \subset \bigcup_{m=0}^{M} \Gamma_{m}$. 
Proof. We organize a stepwise process. The curve $\Gamma$ starts at a point in $\operatorname{Int} B_{j}(j$ is the index of the hole for which we are proving estimate (28)). Put $j_{0}=j$, take the beginning of the curve $\Gamma$ for $z_{0}$, let $w_{0}$ be the point of exit of $\Gamma$ from $B_{j}$, and let $\beta_{0}$ be the arc of $\Gamma$ from $z_{0}$ to $w_{0}$.

Let $m=0,1, \ldots$ Suppose that the index $j_{m}$, the arc $\beta_{m}$, and points $z_{m}, w_{m}$ are already chosen. Consider the disk $\overline{\mathcal{B}}\left(w_{m},\left|z_{m}-w_{m}\right|\right)$. Starting with the point $w_{m}$ and moving along $\Gamma$ in the direction of increase of its parameter, we necessarily escape the disk $\overline{\mathcal{B}}\left(w_{m},\left|z_{m}-w_{m}\right|\right)$, because the end of the polygonal chain $\Gamma$ is situated at the distance 6 from the origin, by our assumptions on $\Gamma$. For $\xi_{m}$ we take the point of the first exit of $\Gamma$ from $\overline{\mathcal{B}}\left(w_{m},\left|z_{m}-w_{m}\right|\right)$; the arc $\Gamma_{m}$ is defined as the arc on $\Gamma$ from $z_{m}$ to $\xi_{m}$.

Recall that $\Gamma$ enters and exits any hole $B_{j^{\prime}}$ no more than once. We check the following cases.

(1) $\xi_{m} \in \operatorname{Int} B_{j^{\prime}}$ for some $j^{\prime}$. Then put $z_{m+1}=\xi_{m}, j_{m+1}=j^{\prime}$. For $w_{m+1}$ we take the point of exit of the chain $\Gamma$ from $B_{j^{\prime}}$. The arc $\beta_{m+1}$ is then defined as a closed arc of $\Gamma$ from $z_{m+1}$ until $w_{m+1}$.

(2) The point $\xi_{m}$ lies in $\Omega$ or on the boundary of one of the holes, but the arc of the chain $\Gamma$ after $\xi_{m}$ does not lie in $\Omega \cup \mathbb{D}^{(c)}$ entirely. Let $B_{j_{m+1}}$ be the first of the holes $B_{j^{\prime}}$ that $\Gamma$ intersects after $\xi_{m}$. We define $z_{m+1}$ as the point of entrance of $\Gamma$ into $B_{j_{m+1}}$. (If $\xi_{m}$ is a point of entrance of $\Gamma$ into some hole $B_{j^{\prime}}$, then put $j_{m+1}:=j^{\prime}, z_{m+1}=\xi_{m}$.) Let $w_{m+1}$ be the point of exit of $\Gamma$ from $B_{j_{m+1}}$. The $\operatorname{arc} \beta_{m+1}$ is defined as the closed arc of $\Gamma$ from $z_{m+1}$ to $w_{m+1}$.

(3) The chain $\Gamma$ after the point $\xi_{m}$ lies in $\Omega \cup \mathbb{D}^{(c)}$ entirely. Then we stop our process by putting $M=m$.

(4) $\xi_{m} \in \mathbb{D}^{(c)}$. In this case we also stop our process and set $M=m$.

Our process must stop, because the number of holes in $\Omega$ is finite by the assumptions made at the beginning of the proof of Lemma 4.3 The same assumptions easily allow us to check all the required properties of the points and arcs constructed.

In the setting of the construction performed in Lemma 4.4 the following estimate is true.

Lemma 4.5. $\mathcal{H}^{1}\left(\Gamma_{m} \cap \Omega\right) \geq e^{-2 \pi \widetilde{C}_{B}^{2}(\Omega)} \cdot\left|z_{m}-w_{m}\right|$ for any $m=0,1, \ldots, M$.

Proof. Let $E_{m}$ be the union of the holes $B_{j^{\prime}}$ intersected by $\Gamma_{m}$ and distinct from $B_{j_{m}}$. If $\xi_{m} \in \mathbb{D}^{(c)}$, then we add $\mathbb{D}^{(c)}$ to that union. By the monotonicity of capacity,

$$
\operatorname{Cap}_{2}\left(B_{j_{m}}, E_{m}\right) \leq \operatorname{Cap}_{2}\left(B_{j_{m}}, \mathbb{D}^{(c)} \cup \bigcup_{j \neq j_{m}} B_{j}\right) \leq \widetilde{C}_{B}^{2}(\Omega) .
$$

This condition implies the existence of a function $f \in W_{\text {loc }}^{1,2}(\mathbb{C})$ such that $f=0$ on $B_{j_{m}}$ and $f=1$ on $E_{m}$ almost everywhere, but $\int_{\mathbb{C}}|\nabla f|^{2} d \lambda_{2} \leq 2 \widetilde{C}_{B}^{2}(\Omega)$.

Put

$$
R_{m}=\left\{r \in\left(0,\left|z_{m}-w_{m}\right|\right): \partial \mathcal{B}\left(w_{m}, r\right) \cap \operatorname{Int} E_{m} \neq \varnothing\right\} .
$$

We may assume that $f$ is absolutely continuous on the circle $\partial \mathcal{B}\left(w_{m}, r\right)$ for almost all $r \in R_{m}$. For all such $r$, the circle $\partial \mathcal{B}\left(w_{m}, r\right)$ intersects the two sets Int $E_{m}$ and Int $B_{j_{m}}$ (because it must contain at least one inner point of the arc $\beta_{m}$, whereas all such points lie in Int $B_{j_{m}}$ in accordance with the assumptions made at the beginning of the proof of Lemma 4.3). Hence, for almost every $r \in R_{m}$, the function $f$ takes values 0 (on $B_{j_{m}}$ ) and 1 (on $\left.E_{m}\right)$ on the circle $\partial \mathcal{B}\left(w_{m}, r\right)$, which gives

$$
\int_{\partial \mathcal{B}\left(w_{m}, r\right)}|\nabla f|^{2} d \mathcal{H}^{1} \geq \frac{1}{\pi r}
$$


by the Cauchy-Schwartz inequality. This implies that

$$
\int_{R_{m}} \frac{d r}{r} \leq 2 \pi \widetilde{C}_{B}^{2}(\Omega)
$$

As a consequence, we have

$$
\frac{\mathcal{H}^{1}\left(R_{m}\right)}{\left|z_{m}-w_{m}\right|} \leq 1-e^{-2 \pi \widetilde{C}_{B}^{2}(\Omega)} .
$$

Indeed, the set $X_{0}=\left[\left|w_{m}-z_{m}\right|-\mathcal{H}^{1}\left(X_{0}\right),\left|w_{m}-z_{m}\right|\right]$ gives minimum to the integral $\int_{X} \frac{d r}{r}$ over all sets with the same length.

Thus, we have proved that

$$
\mathcal{H}^{1}\left(\left[0,\left|w_{m}-z_{m}\right|\right] \backslash R_{m}\right) \geq e^{-2 \pi \widetilde{C}_{B}^{2}(\Omega)} \cdot\left|w_{m}-z_{m}\right| .
$$

Recall that $\Gamma_{m}$ is a polygonal chain with finite number of vertices. Consider the set $A_{m}=\left\{z \in \mathcal{B}\left(w_{m},\left|z_{m}-w_{m}\right|\right):\left|z-w_{m}\right| \notin \operatorname{clos} R_{m}\right\}$; this set is a union of finitely many annuli, the sum of their widths, by (29), is at least $e^{-2 \pi \widetilde{C}_{B}^{2}(\Omega)}\left|z_{m}-w_{m}\right|$, and at the same time $A_{m} \subset \Omega$. The arc $\Gamma_{m} \backslash B_{j_{m}}$ starts at the common center of these annuli and escapes the greatest of them. Thus, $\mathcal{H}^{1}\left(\Gamma_{m} \cap \Omega\right) \geq e^{-2 \pi \widetilde{C}_{B}^{2}(\Omega)}\left|z_{m}-w_{m}\right|$, and the desired estimate is established.

Now we finish the proof of Lemma 4.3. If the point $\xi_{M}$ constructed in Lemma 4.4 lies in $\mathbb{D}^{(c)}$, then shrink the curve $\Gamma$ by cutting from $\Gamma$ its arc after the point $\xi_{M}$. Now we assume that $\Gamma \backslash \bigcup_{m=0}^{M} \Gamma_{m} \subset \Omega$.

The curve $\Gamma$ joins a point on $B_{j}$ with a point on $\mathbb{D}^{(c)}$. Hence,

$$
\mathcal{H}^{1}\left(\Gamma \backslash \bigcup_{m=0}^{M} \Gamma_{m}\right)+\sum_{m=0}^{M} \operatorname{diam}\left(\Gamma_{m}\right) \geq \operatorname{dist}\left(B_{j}, \partial \mathbb{D}\right) .
$$

At the same time, $\Gamma \backslash \bigcup_{m=0}^{M} \Gamma_{m} \subset \Omega$ by Lemma 4.4 Also, by Lemma 4.5,

$$
\mathcal{H}^{1}\left(\Gamma_{m} \cap \Omega\right) \geq \frac{\operatorname{diam}\left(\Gamma_{m}\right)}{2 e^{2 \pi \widetilde{C}_{B}^{2}(\Omega)}} .
$$

Since the curves $\Gamma_{m}, m=0,1, \ldots, M$ are disjoint, we get $\mathcal{H}^{1}(\Gamma \cap \Omega) \geq \frac{\operatorname{dist}\left(B_{j}, \partial \mathbb{D}\right)}{2 e^{2 \pi \widetilde{C}_{B}^{2}(\Omega)}}$. Lemma 4.3 is proved.

Now we finish the proof of Theorem 4.2. The function $u$ was defined above by the metric $\rho$. Also, $u$ is constant on any of the sets $B_{j}$, and the estimate

$$
\left.u\right|_{B_{j}} \geq \frac{\operatorname{dist}\left(B_{j}, \partial \mathbb{D}\right)}{2 e^{2 \pi \widetilde{C}_{B}^{2}(\Omega)}},
$$

is true, whereas the boundary values of $u$ on $\partial \mathbb{D}$ are zero. Moreover, $|\nabla u| \leq 1$ almost everywhere. By the second assertion of Proposition 2.10, we have

$$
\pi \geq\|u\|_{W^{1,2}(\mathbb{D})}^{2} \geq C_{I}^{-2}(\Omega) \cdot \sum_{j=1}^{\infty}\left(\left.u\right|_{B_{j}}\right)^{2} \geq \frac{1}{4 C_{I}^{2}(\Omega) e^{4 \pi \widetilde{C}_{B}^{2}(\Omega)}} \cdot \sum_{j=1}^{\infty} \operatorname{dist}\left(B_{j}, \partial \mathbb{D}\right)^{2},
$$

Moreover, the weak Bessel property implies that the quantities $\operatorname{diam}_{H} B_{j}$ are bounded from above by a constant depending only on $\widetilde{C}_{B}(\Omega)$. In particular, this means that the quantities

$$
\frac{\max \left\{\operatorname{dist}(z, \partial \mathbb{D}): z \in B_{j}\right\}}{\min \left\{\operatorname{dist}(z, \partial \mathbb{D}): z \in B_{j}\right\}}
$$


are bounded by a constant depending only on $\widetilde{C}_{B}(\Omega)$. Since

$$
\sum_{j=1}^{\infty} \operatorname{dist}\left(B_{j}, \partial \mathbb{D}\right)^{2} \leq 4 \pi C_{I}^{2}(\Omega) e^{4 \pi \widetilde{C}_{B}^{2}(\Omega)},
$$

we conclude that the disk $\mathcal{B}\left(0, \frac{1}{2}\right)$ can intersect only finitely many holes $B_{j}$, the number of which can be estimated from above in terms of $C_{I}(\Omega)$ and $\widetilde{C}_{B}(\Omega)$ only. The theorem is proved.

Remark 1. Again, we have obtained the convergence of the series $\sum_{j=1}^{\infty} \operatorname{dist}\left(B_{j}, \partial \mathbb{D}\right)^{2}$, now under weaker assumptions than those in Theorem 3.4. The nonsharpness of the exponent 2 involved in this sum will be discussed in Subsection 6.1.

Remark 2. The Bessel property (or the weak Bessel property) alone or the interpolation property alone do not imply the uniform local finiteness property of $\Omega$.

To construct a domain $\Omega$ with $C_{B}(\Omega)$ not large but with $N(\Omega)$ large, we pick a large number $M \in \mathbb{N}$. For $m=0,1, \ldots, M$, put $B_{m}:=\overline{\mathcal{B}}\left(\frac{m}{2 M}, \frac{1}{10 M}\right)$. Since these holes are $3 / 2$-strongly separated, the Bessel constant of the domain $\Omega_{M}:=\mathbb{D} \backslash \bigcup_{m=0}^{M} B_{m}$ does not exceed some absolute constant (Proposition 2.24). At the same time, $N(\Omega) \geq M$. Acting with conformal shifts of such domains and letting $M \rightarrow \infty$ like in Example 3.3 in Subsection 3.3, one can construct a domain $\Omega$ for which $C_{B}(\Omega)<+\infty$ but $N(\Omega)=+\infty$.

Now we construct a domain $\Omega$ with $C_{I}(\Omega)$ not large but with $N(\Omega)$ large. Again, we pick large $M \in \mathbb{N}$ and also a small $\delta>0$. Next, we take a monotone increasing sequence of real numbers $x_{1}, x_{2}, \ldots, x_{M} \in(-1,1)$ such that for the disks $B_{m}:=\overline{\mathcal{B}}\left(x_{m}, \frac{1}{2 M}\right)$, $m=1,2, \ldots, M$, we have $\operatorname{dist}\left(B_{M}, \partial \mathbb{D}\right)=\delta$, $\operatorname{dist}\left(B_{m}, B_{m+1}\right)=\delta$ for $m=1,2, \ldots, M-1$. To estimate the interpolation constant of the domain $\Omega_{M}:=\mathbb{D} \backslash \bigcup_{m=1}^{M} B_{m}$ from above, we apply Proposition 2.10, Let $u \in \mathcal{A} d m\left(\Omega_{m}\right)$ be an admissible function. Then, by Proposition 2.19, we have the inequalities

$$
\left|\left(\left.u\right|_{B_{M}}\right)\right| \leq C(M, \delta) \cdot\|u\|_{W^{1,2}(\mathbb{D})}, \quad\left|\left(\left.u\right|_{B_{m}}\right)-\left(\left.u\right|_{B_{m+1}}\right)\right| \leq C(M, \delta) \cdot\|u\|_{W^{1,2}(\mathbb{D})}
$$

for $m=1,2, \ldots, M-1$, where $C(M, \delta) \rightarrow 0$ as $\delta \rightarrow 0$ with $M$ fixed. Consecutive application of these estimates for $m=M-1, M-2, \ldots, 2,1$ allows us to conclude that the inequality $\sum_{j=1}^{\infty}\left(\left.u\right|_{B_{j}}\right)^{2} \leq\|u\|_{W^{1,2}(\mathbb{D})}^{2}$ will be true if for fixed $M$ the number $\delta$ is sufficiently small; then $C_{I}\left(\Omega_{m}\right) \leq 1$. On the other hand, if $M$ is large and $\delta$ is small, then we have $N\left(\Omega_{M}\right) \geq M / 2$. To construct a domain $\Omega$ for which $C_{I}(\Omega)<+\infty$ but $N(\Omega)=+\infty$, one may act by conformal shifts of the resulting domains $\Omega_{M}$ and let $M \rightarrow \infty$, as in Example 3.3 in Subsection 3.3 .

Remark 3. In the construction of the function $u$ in the proof of Theorem4.2 we were solving the following extremal problem: to maximize $\sum_{j=1}^{\infty}\left(\left.u\right|_{B_{j}}\right)^{2}$ if $u \in W^{1,2}(\mathbb{D}),\left.u\right|_{\partial \mathbb{D}}=0$, and $|\nabla u| \leq \varphi$ almost everywhere. This was done for $\varphi=\mathbb{1}_{\Omega}$. Such a problem can also be solved in larger generality. First, under very general assumptions there exists a function $u$ giving the maximum to $u(z)$ simultaneously at (almost) all points $z \in \mathbb{D}$. Second, function $\varphi: \mathbb{D} \rightarrow[0,+\infty)$ can be more or less arbitrary. In other words, under a pointwise upper estimate on $|\nabla u|$, one can maximize any norm of $u$ that depends monotonically on its values. Moreover, such a function $u$ can be found as explicitly as this was done in the proof of Theorem 4.2 .

Then the metric $\rho$ can be defined in a similar way: if $z_{1}, z_{2} \in \operatorname{clos} \mathbb{D}$, then

$$
\rho\left(z_{1}, z_{2}\right):=\inf \int_{\Gamma} \varphi d \mathcal{H}^{1}
$$


where the infimum is taken over all curves $\Gamma$ joining $z_{1}$ and $z_{2}$. Next, one may put $u(z):=\operatorname{dist}_{\rho}(z, \partial \mathbb{D}), z \in \mathbb{D}$. Nevertheless, problems concerning the measurability of the integrand in (30) may arise now. Moreover, the function $u$ may collapse. For example, for $x+i y \in \mathbb{D}$ put $\varphi(x+i y):=0$ if $x$ or $y$ is rational and $\varphi(x+i y):=1$ otherwise; then for the metric $\rho$ defined by (30) we have $\rho\left(z_{1}, z_{2}\right)=0$ for all $z_{1}, z_{2} \in \operatorname{clos} \mathbb{D}$, and $u \equiv 0$ in $\operatorname{clos} \mathbb{D}$.

Such difficulties can be overcome by replacing the infimum in (30) by the essential infimum, that is, by the infimum up to a negligible family of curves. Such smallness should be understood in the sense of the module (or extremal length) of the exceptional family, see 14,15. Fortunately, we did not need these techniques: in our case the function $\varphi$ is not very nonsmooth coinciding with the indicator of a locally-smooth set $\Omega$. And the difficulties related to the possible occurrence of nonsmooth curves $\Gamma$ in (30) were overcome by working with finite-verticed polygonal chains.

\section{§5. CRiteria Under the CONDITION OF UNifORM LOCAL FINITENESS}

Our goal is to give a metric criterion of the complete interpolation property. The questions about the description of domains possessing only the Bessel or only the interpolation property seem unreachable. At the same time, such questions can be answered if, in addition, we impose the uniform local finiteness condition on the family of holes $B_{j}$. We have already proved (in Theorem 4.2) that this is the case when $\Omega$ has the complete interpolation property.

5.1. The Bessel property criterion. Recall that the notion of the weak separation of holes was introduced in Definition 2.18 Propositions 2.19 and 2.15$]$ imply that the weak (or strong) Bessel property implies the weak separation of holes. In this subsection we shall prove the reverse implication under the condition of uniform local finiteness.

Theorem 5.1. Let $\Omega$ be a regular domain, and let $B_{j}$ be holes in $\Omega$. Suppose that $\sup _{j \in \mathbb{N}} \operatorname{diam}_{H}\left(B_{j}\right)<+\infty, N(\Omega)<+\infty$ (that is, any disk of the form $\mathcal{B}_{H}(z, 1)$ intersects at most $N(\Omega)$ holes $B_{j}$ ) and that the holes $B_{j}$ are $\varepsilon$-weakly separated for some $\varepsilon>0$. Then $\Omega$ possesses the Bessel property, and the Bessel constant $C_{B}(\Omega)$ can be estimated from above in terms of $\sup _{j \in \mathbb{N}} \operatorname{diam}_{H}\left(B_{j}\right), N(\Omega)$, and $\varepsilon$ only.

First we prove the following statement.

Proposition 5.2. The assumptions of Theorem 5.1 ensure the weak Bessel property of $\Omega$. Moreover, the constant $\widetilde{C}_{B}(\Omega)$ can be estimated from above only in terms of the constants occurring in that theorem.

Proof. Indeed, Proposition 2.15 shows that we need to get an upper estimate for the capacity $\operatorname{Cap}_{2}\left(B_{j}, \Omega^{(c)} \backslash B_{j}\right), j=1,2, \ldots$ Recall that the condition

$$
\sup _{j \in \mathbb{N}} \operatorname{diam}_{H}\left(B_{j}\right)<+\infty
$$

means that the quantities $\frac{\operatorname{dist}\left(B_{j}, \partial \mathbb{D}\right)}{\operatorname{diam}\left(B_{j}\right)}$ are separated away from zero by some constant $c>0$. Let $\left\{B_{j^{\prime}}\right\}_{j^{\prime} \in J}$ be the set of holes $B_{j^{\prime}}\left(j^{\prime} \neq j\right)$ for which

$$
\operatorname{dist}\left(B_{j}, B_{j^{\prime}}\right) \leq(c / 2) \cdot \operatorname{diam}\left(B_{j}\right)
$$

Let $U_{j}$ be the $\left(c \cdot \operatorname{diam}\left(B_{j}\right) / 2\right)$-neighborhood of $B_{j}$. The quantity $\operatorname{diam}_{H} U_{j}$ is finite and can be estimated from above via $c$; hence, by the uniform local finiteness, the set of indices $J$ is finite, and the number of its elements can be estimated in terms of the constants in the assumptions. The capacity $\operatorname{Cap}_{2}\left(B_{j}, \mathbb{D}^{(c)} \cup \bigcup_{j^{\prime} \notin J, j^{\prime} \neq j} B_{j^{\prime}}\right)$ is not greater than $\operatorname{Cap}_{2}\left(B_{j}, U_{j}^{(c)}\right)$. As it was proved in Proposition 2.22, the last capacity is bounded 


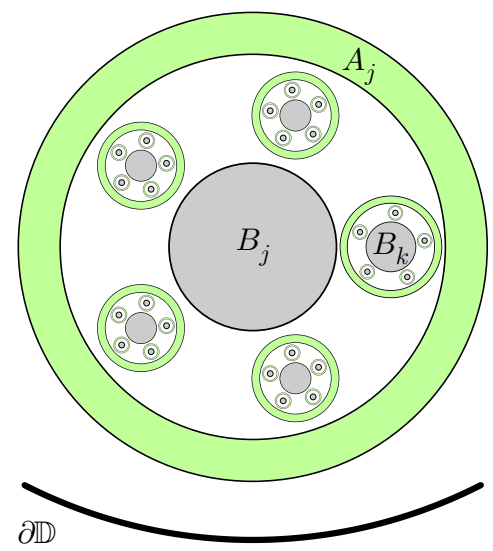

Figure 10. Partial order on the set of holes.

from above by a value depending only on $c$. Moreover, for any $j^{\prime} \in J$ the capacity $\mathrm{Cap}_{2}\left(B_{j}, B_{j^{\prime}}\right)$ is bounded from above by a value depending only on the constant $\varepsilon$ of weak separation of holes (Corollary 2.23). Now the estimate on card $J$ and the semiadditivity of the capacity $\mathrm{Cap}_{2}(\cdot, \cdot)$ with respect to the second argument imply the desired upper estimate for $\operatorname{Cap}_{2}\left(B_{j}, \Omega^{(c)} \backslash B_{j}\right)$.

Proof of Theorem 5.1. By Proposition 5.2 proved above, the quantity $\widetilde{C}_{B}(\Omega)$ is finite and can be estimated in terms of the constants occurring in the assumptions of the theorem. But by Theorem 2.16 we have $C_{B}(\Omega) \leq \sqrt{2} \cdot \widetilde{C}_{B}(\Omega)$, which concludes the proof.

Theorem 5.1 can be also proved without application of Theorem 2.16 and in terms of metric characteristics of domain $\Omega$ only. It is interesting that a discrete partial order structure on the set of holes arises naturally in such a proof.

For $a>0$ denote by $U_{a}\left(B_{j}\right)$ the closed $a$-neighborhood of the set $B_{j}$. Put $\Lambda_{j}:=$ $\operatorname{diam} B_{j}$.

Lemma 5.3 below, in fact, gives a description of the domains satisfying the assumptions of Theorem 5.1. Figure 10 illustrates the construction in that lemma: the disks depicted in this draft are the holes $B_{j}$, and the sets $A_{j}$ from Lemma 5.3 are annuli concentric with the disks $B_{j}$ corresponding to them.

Lemma 5.3. In the settings of Theorem 5.1, it is possible to define a strict partial order relation $\succ$ on the set of holes $B_{j}$ and also to associate a set $A_{j} \subset \mathbb{D}$ with each hole $B_{j}$, $j=1,2, \ldots$, so as to satisfy the following conditions.

(1) For each $j=1,2, \ldots$, the set $A_{j}$ is of the form $U_{s_{j}}\left(B_{j}\right) \backslash U_{t_{j}}\left(B_{j}\right)$ for some $t_{j}, s_{j} \in(0,+\infty)\left(s_{j}>t_{j}\right)$. Also, $s_{j}-t_{j} \geq c_{2} \Lambda_{j}$ and $s_{j} \leq c_{1} \Lambda_{j}$, where the constants $c_{1}, c_{2} \in(0,+\infty)$ do not depend on $j$ (and can be estimated only in terms of the constants from the hypothesis of Theorem [5.1). Moreover, $A_{j} \subset \Omega$.

(2) The overlap multiplicity of the sets $A_{j}$ does not exceed some constant $C_{1}<+\infty$.

(3) $B_{j^{\prime}} \prec B_{j}\left(j^{\prime} \neq j\right)$ if and only if $U_{s_{j^{\prime}}}\left(B_{j^{\prime}}\right) \subset U_{s_{j}}\left(B_{j}\right)$, and also if and only if $B_{j^{\prime}} \subset U_{t_{j}}\left(B_{j}\right)$.

(4) For a fixed $j_{0} \in \mathbb{N}$, the number of indices $j$ for which $B_{j} \prec B_{j_{0}}$ does not exceed some constant $C$. In particular, the lengths of the chains with respect to the order $\succ$ are upper bounded. If $B_{j_{1}}, B_{j_{2}} \succ B_{j}$, then either $B_{j_{1}} \succ B_{j_{2}}$, or $B_{j_{2}} \succ B_{j_{1}}$.

The constants $c_{1}, c_{2}, C, C_{1}$ depend only on constants from Theorem 5.1 
The proof is given in the Appendix. From this proof it is seen that we may force $c_{1}$ to be arbitrarily small. Therefore, the relation $B_{k} \prec B_{j}$ for some $j, k=1,2, \ldots$ means, roughly speaking, that $\operatorname{diam} B_{k} \ll \operatorname{diam} B_{j}$ and $\operatorname{dist}\left(B_{j}, B_{k}\right) \ll \operatorname{diam} B_{j}$.

Remark. The fourth assertion of Lemma 5.3 allows us to endow the set of holes with the structure of an oriented forest.

Namely, let $\mathcal{M}$ denote the set of indices of the maximal elements in the order $\succ$. Let $j \notin \mathcal{M}$. By the fourth assertion of Lemma 5.3. the set $\left\{B_{j^{\prime}}: B_{j^{\prime}} \succ B_{j}\right\}$ is finite and is a chain in the order $\succ$. Therefore, there exists a unique minimal element $B_{j^{\prime \prime}}$ in this set; we denote $j^{\prime \prime}$ by $\mathcal{P}(j)$. Thus, we have defined "the nearest ancestor" mapping $\mathcal{P}: \mathbb{N} \backslash \mathcal{M} \rightarrow \mathbb{N}$.

Let us define an oriented graph $\mathscr{G}$. Its vertices will be all the holes $B_{j}, j \in \mathbb{N}$. For each $j \in \mathbb{N} \backslash \mathcal{M}$, we draw an edge in the graph $\mathscr{G}$ from $B_{\mathcal{P}(j)}$ to $B_{j}$. Let $\tilde{\mathscr{G}}$ be the nonoriented graph obtained by forgetting the directions of the edges in $\mathscr{G}$. The fourth assertion of Lemma 5.3 allows us to conclude that the graph $\tilde{\mathscr{G}}$ is a forest, that is, a countable disjoint union of trees. Also, the numbers of vertices in the connected components of $\tilde{\mathscr{G}}$ are bounded from above. In any of such components, one vertex has zero incoming degree in the graph $\mathscr{G}$, whereas all other vertices in that component have incoming degree 1.

Suppose that all holes $B_{j}$ are disks. Then any set $A_{j}, j=1,2, \ldots$, constructed in Lemma 5.3 is an annulus; $A_{j}$ winds exactly around those holes $B_{j^{\prime}}$ for which $B_{j^{\prime}} \preceq B_{j}$. By the first assertion of Lemma 5.3 , the annulus $A_{j}$ is sufficiently wide, and if $\omega \in L_{c}^{2,1}(\Omega)$, then

$$
\int_{A_{j}}|\omega|^{2} d \lambda_{2} \geq c \cdot\left(\sum_{j^{\prime}: B_{j^{\prime}} \preceq B_{j}} \operatorname{Per}_{j^{\prime}} \omega\right)^{2},
$$

with some $c>0$ independent of $j$ and $\omega$. We apply this inequality consecutively, starting with the minimal holes in the order $\succ$ and up to the maximal ones. Recalling that the lengths of the chains in the order $\succ$ are bounded, as well as the overlap multiplicity of the sets $A_{j}$, we can estimate $\|$ Per $\omega \|_{\ell^{2}}$ from above in terms of $\|\omega\|_{L_{c}^{2,1}(\Omega)}$.

Now, let the holes $B_{j}$ be not necessarily disks. In this case, Theorem 5.1 can be derived from Lemma 5.3 with the help of Proposition 2.10. Let us sketch this proof.

By the first assertion of Lemma 5.3 and Proposition [2.22, for any $j=1,2, \ldots$ there exists a function $u_{j} \in \stackrel{\circ}{W}^{1,2}(\mathbb{D})$ such that $u_{j}=0$ in $\mathbb{R}^{2} \backslash U_{s_{j}}\left(B_{j}\right), u_{j}=1$ in $U_{t_{j}}\left(B_{j}\right)$, $\left\|u_{j}\right\|_{W^{1,2}(\mathbb{D})} \leq C_{2}$, here the constant $C_{2}<+\infty$ depends only on the numbers $c_{1}$ and $c_{2}$ constructed in Lemma 5.3. Let $\left\{a_{j}\right\}_{j=1}^{\infty}$ be a finitely supported sequence of real numbers. For this sequence, we construct a function $u$ enjoying the conditions of the first assertion in Proposition 2.10, Put

$$
u(z):=\sum_{j \in \mathcal{M}} a_{j} u_{j}(z)+\sum_{j \notin \mathcal{M}}\left(a_{j}-a_{\mathcal{P}(j)}\right) u_{j}(z), \quad z \in \mathbb{D}
$$

(the set $\mathcal{M}$ of indices of the maximal elements and the nearest ancestor mapping $\mathcal{P}$ have been introduced above). The third and fourth assertions of Lemma 5.3 allow us to show that $\left.u\right|_{B_{j}}=a_{j}$ for any $j=1,2, \ldots$, whereas the second and fourth assertions of that lemma provide the estimate $\|u\|_{W^{1,2}(\mathbb{D})} \leq C_{3} \cdot\left\|\left\{a_{j}\right\}_{j=1}^{\infty}\right\|_{\ell^{2}}$ with some $C_{3}<+\infty$ depending only on the constants occurring in the assumptions of Theorem 5.1. Now Theorem 5.1 follows from Proposition 2.10.

5.2. Interpolation criterion. Now our goal is to establish a necessary and sufficient condition for interpolation if the uniform local finiteness of the family of holes $B_{j}$ occurs. For this, we shall need a graph. 
Pick a number $S \in(0,+\infty)$. We define a nonoriented graph $G(\Omega, S)$ as follows. The vertices of $G(\Omega, S)$ will be all the connected components of $\Omega^{(c)}$, that is, all the holes $B_{j}$, $j=1,2, \ldots$, and also the set $\mathbb{D}^{(c)}$. Two holes $B_{j_{1}}$ and $B_{j_{2}}$ are joined by an edge in the graph $G(\Omega, S)$ if $\operatorname{dist}\left(B_{j_{1}}, B_{j_{2}}\right) \leq S \cdot \min \left\{\operatorname{diam} B_{j_{1}}, \operatorname{diam} B_{j_{2}}\right\}$. Next, we join $B_{j}$ and $\mathbb{D}^{(c)}$ by an edge if $\operatorname{dist}\left(B_{j}, \mathbb{D}^{(c)}\right) \leq S \cdot \operatorname{diam} B_{j}$. If $S$ increases, then so does the set of edges in this graph.

We define the distance between two vertices in a (nonoriented) graph $G$ as the number of edges of the shortest path joining these vertices (thus, the distance between two adjacent vertices is 1); if there is no such path, then the distance is set to be infinite. Let dist $_{G}$ denote the distance in $G$.

The capacity connectedness property, which we mentioned in the Introduction, is the connectedness of the graph $G(\Omega, S)$ for some $S<+\infty$, together with the finiteness of its diameter in the metric $\operatorname{dist}_{G(\Omega, S)}$; the latter condition will often be understood as the estimate $\sup _{j \in \mathbb{N}} \operatorname{dist}_{G(\Omega, S)}\left(B_{j}, \mathbb{D}^{(c)}\right)<+\infty$.

Remark. Let $\Omega$ be a regular domain; recall that in this case any set $B_{j}, j=1,2, \ldots$, is the closure of a domain with smooth boundary. We may define another graph $g(\Omega, s)$, where $s>0$. Like in the case of $G(\Omega, S)$, its vertices will be all the connected components of $\Omega^{(c)}$. Two such vertices $E_{1}$ and $E_{2}$ are joined by an edge in the graph $g(\Omega, s)$ if $\operatorname{Cap}_{2}\left(E_{1}, E_{2}\right) \geq s$. By Proposition 2.19, if the graph $G(\Omega, S)$ is connected or its diameter admits an upper estimate, then, for some $s>0$ depending only on $S$, the graph $g(\Omega, s)$ will be connected (or, respectively, its diameter will admit an upper estimate). By Corollary 2.23, the converse is also true: the connectedness (or boundedness of the diameter) of the graph $g(\Omega, s)$ implies the connectedness (respectively, boundedness of the diameter) of the graph $G(\Omega, S)$ for some $S<+\infty$ depending only on $s$.

In this subsection we shall mainly deal with the graph $g(\Omega, s)$, using its conformal invariance. The graph $G(\Omega, S)$ will be appropriate for criteria in the case where the holes $B_{j}$ have nonsmooth boundaries (Subsection 6.2).

Theorem 5.4. Let $\Omega$ be a regular domain and suppose that $N(\Omega)<+\infty$ (i.e., the family of holes in $\Omega$ is uniformly locally finite). If $\Omega$ possesses the interpolation property, then there exists $S<+\infty$ and $M \in \mathbb{N}$ such that the graph $G(\Omega, S)$ is connected and for any hole $B_{j}$ the distance from $B_{j}$ to $\mathbb{D}^{(c)}$ in the graph $G(\Omega, S)$ is at most $M$.

The numbers $S$ and $M$ can be estimated from above in terms of $C_{I}(\Omega)$ and $N(\Omega)$.

Remark. As it will be seen from the argument below, for appropriate $S$ we may take $M=N(\Omega)+1$.

Proof of Theorem 5.4. The idea of the argument below can be briefly sketched as follows. Suppose that $r<\frac{1}{4}$, and let $\mathcal{C}$ be a connected component of the graph $G(\Omega, S)$ such that all vertices in $\mathcal{C}$ lie in $\mathcal{B}(0, r)$. Assume also that the annulus $\mathcal{B}\left(0, \frac{1}{2}\right) \backslash \mathcal{B}(0, r)$ lies in $\Omega$. Suppose that either $r$ is small, or the number of vertices in $\mathcal{C}$ is large. The function $u$ for which $\left.u\right|_{\mathcal{B}(0, r)}=1,\left.u\right|_{\mathbb{C} \backslash \mathcal{B}\left(0, \frac{1}{2}\right)}=0$, and $\Delta u=0$ in $\mathcal{B}\left(0, \frac{1}{2}\right) \backslash \overline{\mathcal{B}}(0, r)$ is admissible for $\Omega$. Applying the second assertion of Proposition 2.10 to $u$, we conclude that the constant $C_{I}(\Omega)$ is large, which contradicts the hypothesis of the theorem.

Now we pass to the formal argument. By the remark before the theorem, it suffices to check the connectedness and the boundedness of the diameter of $g(\Omega, s)$ for some $s>0$ depending only on $N(\Omega)$ and $C_{I}(\Omega)$.

Let $N=N(\Omega)$. Put $\widetilde{s}=C_{I}^{-2}(\Omega) / 2 N$. Choose $F=F(\widetilde{s})$ such that if $\operatorname{Cap}_{2}\left(B_{j}, B_{j^{\prime}}\right) \geq \widetilde{s}$, then $\operatorname{dist}\left(B_{j}, B_{j^{\prime}}\right) \leq F \cdot \min \left\{\operatorname{diam} B_{j}, \operatorname{diam} B_{j^{\prime}}\right\}$ (this can be done by Corollary 2.23); we may assume that $F \geq 1$. Pick a number $\delta>0$ such that $3 \delta N F<\tanh 1$ and also such that if $B_{j} \subset \mathcal{B}(0,3 N F \delta)$, then $\operatorname{Cap}_{2}\left(B_{j},(\mathcal{B}(0, \tanh 1))^{(c)}\right) \leq C_{I}^{-2}(\Omega) / 2$. Finally, 
we pick a positive number $s \leq \widetilde{s}$ such that if $\operatorname{diam} B_{j} \geq \delta$, then $\operatorname{Cap}_{2}\left(B_{j}, \mathbb{D}^{(c)}\right) \geq s$. By construction, $s$ depends only on $N$ and $C_{I}(\Omega)$. We show that the graph $g(\Omega, s)$ is connected and its diameter does not exceed $2 N+2$.

Take any hole $B_{j_{1}}$. We are going to prove that $\operatorname{dist}_{g(\Omega, s)}\left(B_{j_{1}}, \mathbb{D}^{(c)}\right) \leq N+1$. The uniform local finiteness property, the interpolation property, and the graph $g(\Omega, s)$ are invariant under the Möbius transformations, which allows us to assume that $0 \in B_{j_{1}}$. Let $\mathcal{C}$ be the connected component of a vertex $B_{j_{1}}$ in the graph $g(\Omega, s)$ and $\widetilde{\mathcal{C}}$ the connected component of $B_{j_{1}}$ in the graph $g(\Omega, \widetilde{s})$ (this is a subgraph in $g(\Omega, s)$ ). We have $\widetilde{\mathcal{C}} \subset \mathcal{C}$. We are going to prove the following: one of the vertices $B_{j} \in \widetilde{\mathcal{C}}$ is adjacent to $\mathbb{D}^{(c)}$ in $g(\Omega, s)$. Suppose the contrary.

Let $B_{j} \in \widetilde{\mathcal{C}}$. Then $\operatorname{diam} B_{j} \leq \delta$ (otherwise $\operatorname{Cap}_{2}\left(B_{j}, \mathbb{D}^{(c)}\right) \geq s$ by the choice of $s$, and then the vertices $B_{j}$ and $\mathbb{D}^{(c)}$ are adjacent in the graph $g(\Omega, s)$ what contradicts our assumption). Now we will work with the graph $g(\Omega, \widetilde{s})$.

Let vertices $B_{j}, B_{j^{\prime}} \in \widetilde{\mathcal{C}}$ be adjacent in the graph $g(\Omega, \widetilde{s})$, then $\operatorname{Cap}_{2}\left(B_{j}, B_{j^{\prime}}\right) \geq \widetilde{s}$, whence

$$
\operatorname{dist}\left(B_{j}, B_{j^{\prime}}\right) \leq F \cdot \min \left\{\operatorname{diam} B_{j}, \operatorname{diam} B_{j^{\prime}}\right\} \leq \delta F
$$

(the first inequality is true by the choice of $F$, while the second by the estimate for diameters proved above). If $\widetilde{\mathcal{C}}$ contains a simple path of length $N+1$ consisting of edges of $g(\Omega, \widetilde{s})$ and starting in $B_{j_{1}}$, then all $B_{j}$ 's in this path lie in $\mathcal{B}(0,(N+1) \delta+N \delta F)$, because the diameters of the sets $B_{j}$ in this path do not exceed $\delta$, while the distances between adjacent sets do not exceed $\delta F$ (recall that $0 \in B_{j_{1}}$ ). By the choice of $\delta$ we have $(N+1) \delta+N \delta F \leq \tanh 1$. But by the definition of $N$ the disk $\mathcal{B}(0, \tanh 1)$ cannot intersect more than $N$ holes $B_{j}$. Hence $\widetilde{\mathcal{C}}$ cannot contain simple paths starting in $B_{j_{1}}$, going by edges of $g(\Omega, \widetilde{s})$, and having length greater than $N$. This means that any vertex $B_{j} \in \widetilde{\mathcal{C}}$ can be joined in $g(\Omega, \widetilde{s})$ with $B_{j_{1}}$ by a path of length not exceeding $N$. Then, like in the estimate for distances worked out above, we have $B_{j} \subset \mathcal{B}(0, N \delta+(N-1) \delta F) \subset$ $\mathcal{B}(0,3 \delta F N) \subset \mathcal{B}(0, \tanh 1)$. This implies that the number of vertices in $\widetilde{\mathcal{C}}$ is finite and is at most $N$. So, let $\widetilde{\mathcal{C}}=\left\{B_{j_{1}}, B_{j_{2}}, \ldots, B_{j_{K}}\right\}, K \leq N$.

Let $J=\left\{j \in \mathbb{N}: j \neq j_{k}, k=1,2, \ldots, K\right\}$. The interpolation property of the domain $\Omega$ implies that

$$
\mathrm{Cap}_{2}\left(B_{j_{1}} \cup B_{j_{2}} \cup \cdots \cup B_{j_{K}}, \mathbb{D}^{(c)} \cup \bigcup_{j \in J} B_{j}\right) \geq K \cdot C_{I}^{-2}(\Omega) .
$$

Indeed, it suffices to apply the second assertion of Proposition 2.10 to the functions under the infimum in the definition of capacity on the left-hand side of the last inequality (see Definition 2.12). The semiadditivity of capacity (Proposition 2.13) allows us to conclude that there exists $k=1,2, \ldots, K$ for which

$$
\mathrm{Cap}_{2}\left(B_{j_{k}}, \mathbb{D}^{(c)} \cup \bigcup_{j \in J} B_{j}\right) \geq C_{I}^{-2}(\Omega) .
$$

It has already been proved that $B_{j_{k}} \subset \mathcal{B}(0,3 \delta F N)$. If $\left\{B_{j}\right\}_{j \in J_{1}}$ is the set of holes lying outside of $\mathcal{B}(0, \tanh 1)$, then

$$
\mathrm{Cap}_{2}\left(B_{j_{k}}, \mathbb{D}^{(c)} \cup \bigcup_{j \in J_{1}} B_{j}\right) \leq \frac{C_{I}^{-2}(\Omega)}{2 N}
$$

by the choice of $\delta$. Thus, again by the semiadditivity of capacity,

$$
\mathrm{Cap}_{2}\left(B_{j_{k}}, \bigcup_{j \notin J_{1} \cup J} B_{j}\right) \geq \frac{C_{I}^{-2}(\Omega)}{2} .
$$


In the capacity occurring in the last inequality, the second plate of the corresponding condenser is formed by the holes $B_{j} \notin \widetilde{\mathcal{C}}$ that intersect $\mathcal{B}(0, \tanh 1)$. The number of such holes is at most $N$. Hence, for one of them, say $B_{\alpha}$, the following inequality should be true:

$$
\operatorname{Cap}_{2}\left(B_{j_{k}}, B_{\alpha}\right) \geq \frac{C_{I}^{-2}(\Omega)}{2 N}=\widetilde{s} .
$$

But, by the definition of the graph $g(\Omega, \widetilde{s})$, in this case the vertices $B_{j_{k}}$ and $B_{\alpha}$ are adjacent in the graph $g(\Omega, \widetilde{s})$, so that $B_{\alpha} \in \widetilde{\mathcal{C}}$, a contradiction. So, we have proved that the graph $g(\Omega, \widetilde{s})$ contains a path starting at $B_{j_{1}}$ and such that its last vertex is adjacent to $\mathbb{D}^{(c)}$ in the graph $g(\Omega, s)$ (in particular, the graph $g(\Omega, s)$ is connected).

Suppose that $\operatorname{dist}_{g(\Omega, s)}\left(B_{j_{1}}, \mathbb{D}^{(c)}\right)>N+1$. Consider the shortest simple path in the graph $g(\Omega, \widetilde{s})$ from $B_{j_{1}}$ to some $B_{\alpha}$ adjacent to $\mathbb{D}^{(c)}$ in the graph $g(\Omega, s)$. By assumption, the length of this path is at least $N+2$. Consider its first $N+1$ vertices, denoting them by $B_{j_{1}}, B_{j_{2}}, \ldots, B_{j_{N+1}}$. If for some of them we have diam $B_{j_{k}} \geq \delta(k=1,2, \ldots, N+1)$, then in the graph $g(\Omega, s)$ the vertex $B_{j_{k}}$ is adjacent to $\mathbb{D}^{(c)}$ by the choice of $s$. Then the distance in $g(\Omega, s)$ from $B_{j_{1}}$ to $\mathbb{D}^{(c)}$ is at most $N+1$, as required. If $\operatorname{diam} B_{j}<\delta$ for all holes of the path $\left(B_{j_{1}}, B_{j_{2}}, \ldots, B_{j_{N+1}}\right)$, then, by the choice of $F$, the Euclidean distance between adjacent vertices of this path does not exceed $F \delta$ (because $B_{j_{k}}$ is adjacent to $B_{j_{k+1}}$ in the graph $g(\Omega, \widetilde{s}), k=1,2, \ldots, N)$. In this case $B_{j_{k}} \subset \mathcal{B}(0,3 N F \delta) \subset \mathcal{B}(0, \tanh 1)$ for all $k=1,2, \ldots, N+1$. But the disk $\mathcal{B}(0, \tanh 1)$ cannot intersect more than $N$ holes. We have arrived at a contradiction again.

So, under our choice of $s$, the distance from any vertex of the graph $g(\Omega, s)$ to the vertex $\mathbb{D}^{(c)}$ does not exceed $N+1$, and the diameter of this graph is at most $2 N+2$, as claimed. The theorem is proved.

Now we are going to prove the converse: if the diameter of the graph $G(\Omega, S)$ is finite, then $\Omega$ possesses the interpolation property. Our argument can be simplified if we add the estimate $\sup _{j \in \mathbb{N}} \operatorname{diam}_{H}\left(B_{j}\right)<+\infty$ to the hypothesis of the following theorem.

Theorem 5.5. Suppose that the family of holes in a regular domain $\Omega$ is uniformly locally finite. Also, suppose that, for some $S<+\infty$, the graph $G(\Omega, S)$ is connected and that $\operatorname{dist}_{G(\Omega, S)}\left(B_{j}, \mathbb{D}^{(c)}\right) \leq M$ for any $B_{j}$ and some $M \in \mathbb{N}$ independent of $j$. Then the domain $\Omega$ has the interpolation property, and moreover, the constant $C_{I}(\Omega)$ can be estimated from above in terms of $S, M$, and $N(\Omega)$ only.

Proof. First, we sketch the proof. In accordance with Proposition 2.10, we need to prove that for any function $u \in \mathcal{A} d m(\Omega)$ (that is, recall, any function of class $\mathscr{W}^{1,2}(\mathbb{D})$ that is constant on any hole $B_{j}$ ) the following estimate is true:

$$
\int_{\mathbb{D}}|\nabla u|^{2} d \lambda_{2} \geq C_{I}^{-2}(\Omega) \cdot \sum_{j=1}^{\infty}\left(\left.u\right|_{B_{j}}\right)^{2} .
$$

If $B_{j}$ and $B_{k}$ are joined by an edge in $G(\Omega, S)$, then we shall draw a "road" $A_{j k}$ between $B_{j}$ and $B_{k}$ that is sufficiently wide to ensure that

$$
\left|\left(\left.u\right|_{B_{j}}\right)-\left(\left.u\right|_{B_{k}}\right)\right|^{2} \leq C \cdot \int_{A_{j k}}|\nabla u|^{2} d \lambda_{2}
$$

(the constant $C<+\infty$ depends only on the constants in the assumptions of the theorem but not on $j, k$, and $u$.) This construction is illustrated in Figure 11] in this draft, all the holes $B_{j}$ are disks, and the "road" $A_{j k}$ joining $B_{j}$ and $B_{k}$ is $\operatorname{conv}\left(B_{j} \cup B_{k}\right) \backslash$ $\left(B_{j} \cup B_{k}\right)$, where conv $X$ is the convex hull of a set $X \subset \mathbb{R}^{2}$. (The construction below will be performed slightly differently.) Almost all $A_{j k}$ have bounded overlap multiplicity. 


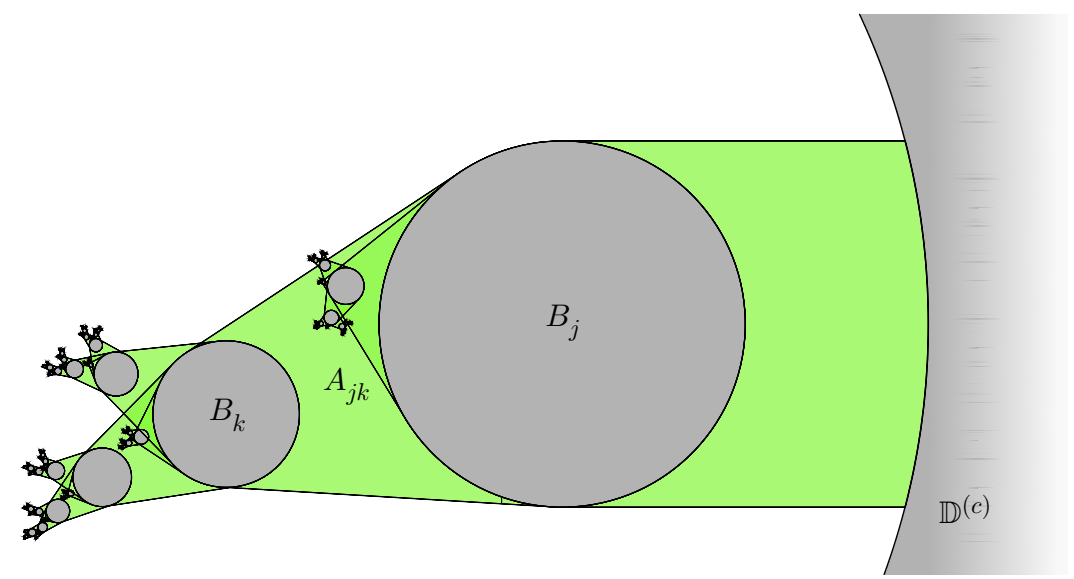

FiguRE 11. Spanning tree of the graph $G(\Omega, s)$

Moreover, the diameter of $G(\Omega, S)$ is finite. Starting with the vertex $\mathbb{D}^{(c)}$, passing over the graph in the breadth-first order, and applying estimates (31) consecutively, we get the desired inequality.

Arguing in such a manner, we shall need, in particular, "roads" joining some holes $B_{j}$ with $\mathbb{D}^{(c)}$. Unfortunately, the overlap multiplicity of such "roads" may turn out to be unbounded. Thus, we need to estimate $\left.u\right|_{B_{j}}$ for such holes $B_{j}$ separately. If a hole $B_{j}$ is adjacent to $\mathbb{D}^{(c)}$ in the graph $G(\Omega, S)$, then $\operatorname{diam}_{H}\left(B_{j}\right) \geq c$, where $c>0$ and depends only on $S$. But in the case of such holes we may argue as in Theorems 3.2 and 3.3 .

Now we pass to the formal argument. In this proof, the constants $C, C_{1}, C_{2}, \cdots \in$ $(0,+\infty)$ depend only on the constants $S, M$, and $N(\Omega)$.

By the remark before Theorem [5.4, there exists $s>0$ depending only on $S$ and such that the graph $g(\Omega, s)$ is connected and $\operatorname{dist}_{g(\Omega, s)}\left(B_{j}, \mathbb{D}^{(c)}\right) \leq M$ for any $B_{j}$. It will be more convenient to work with this graph, using the conformal invariance of capacity.

Fix a function $u \in \mathcal{A} d m(\Omega)$. Put $a_{j}:=\left.u\right|_{B_{j}}, j=1,2, \ldots$ We need to show that

$$
\sum_{j=1}^{\infty} a_{j}^{2} \leq C \cdot\|u\|_{W^{1,2}(\mathbb{D})}^{2}
$$

with $C<+\infty$ depending only on constants from the assumptions but not on $u$.

By Corollary 2.23, there exists a number $F=F(s)$ such that if $B_{j}$ and $B_{k}$ are adjacent in $g(\Omega, s)$, i.e., $\operatorname{Cap}_{2}\left(B_{j}, B_{k}\right) \geq s$, then $\operatorname{dist}\left(B_{j}, B_{k}\right) \leq F \cdot \min \left\{\operatorname{diam} B_{j}\right.$, $\left.\operatorname{diam} B_{k}\right\}$. Let $\delta \in\left(0, \frac{1}{3}\right]$ be sufficiently small. We require the following. First, if $B_{j}$ is adjacent in $g(\Omega, s)$ to $\mathbb{D}^{(c)}$, then $\operatorname{diam}_{H}\left(B_{j}\right) \geq \delta$. Second, if $B_{j} \subset \mathcal{B}(0, \delta)$ and $B_{k} \cap \mathcal{B}\left(0, \frac{1}{3}\right)=\varnothing$, then $\mathrm{Cap}_{2}\left(B_{j}, B_{k}\right)<s$ (and hence $B_{j}$ and $B_{k}$ are not adjacent in the graph $g(\Omega, s)$ ). Third, $\delta(F+2)<\frac{1}{3}$.

Denote by $J_{1}^{\delta}$ the set of indices $j=1,2, \ldots$ for which $\operatorname{diam}_{H}\left(B_{j}\right) \geq \delta$. Next, denote by $J_{2}^{\delta}$ the set of indices $j \notin J_{1}^{\delta}$ for which $B_{j}$ is adjacent in $g(\Omega, s)$ to one of the vertices $B_{k}, k \in J_{1}^{\delta}$. (We could add in $g(\Omega, s)$ all the edges from vertices $B_{j}, j \in J_{1}^{\delta}$, to $\mathbb{D}^{(c)}$ and arrange all holes by their distances from $\mathbb{D}^{(c)}$ in the resulting graph. Yet, we shall not use all the advantages of this trick.)

Recall that if $Z$ is a finite set, then the symbol card $Z$ denotes the number of its elements. 
Lemma 5.6. Let $\left\{E_{j}\right\}_{j \in J}$ be a finite or countable family of compact connected disjoint sets in the disk $\mathbb{D}$. We assume that any $E_{j}, j \in J$, is the closure of a domain with $C^{\infty}$-smooth boundary. Suppose that $\operatorname{diam}_{H}\left(E_{j}\right) \geq \delta$ for all $j \in J$, where $\delta \in\left(0, \frac{1}{3}\right]$.

Suppose also that we are given a finite or countable set $X \subset \bigcup_{j \in J} E_{j}$ for which $n_{j}:=\operatorname{card}\left(X \cap B_{j}\right)<+\infty$ for any $j \in J$. Assume that the set $X$ is uniformly locally finite in the following sense: any hyperbolic disk of radius 1 contains at most $\nu$ points of the set $X$, where $\nu \in \mathbb{N}$ is constant.

Suppose that a function $u \in \stackrel{\circ}{W}^{1,2}(\mathbb{D})$ is constant almost everywhere on any set $E_{j}$, $j \in J$, and equals some number $a_{j} \in \mathbb{R}$ on this set. Then

$$
\sum_{j \in J} n_{j} a_{j}^{2} \leq \widetilde{C}_{1} \cdot\|u\|_{W^{1,2}(\mathbb{D})}^{2},
$$

where $\widetilde{C}_{1}$ depends only on $\nu$ and $\delta$.

Proof. Taking any $z \in X$, we denote by $E(z)$ the set $E_{j}$ containing $z$. Put $K(z):=$ $\mathcal{B}_{H}(z, \delta / 2)$. The hypothesis of lemma implies that the disks $K(z), z \in X$, have overlap multiplicity not exceeding $\nu$ (because $\delta \leq \frac{1}{3}$ ). Let us bring into consideration the hyperbolic area

$$
d A=\frac{4 d \lambda_{2}}{\left(1-|z|^{2}\right)^{2}} \asymp \frac{d \lambda_{2}}{(1-|z|)^{2}} .
$$

This area is invariant under the Möbius transformations. We prove that there exists a constant $\widetilde{C}_{2}>0$ depending only on $\delta$ for which

$$
\left(\left.u\right|_{E(z)}\right)^{2} \leq \widetilde{C}_{2} \cdot\left(\int_{K(z)} u^{2} d A+\int_{K(z)}|\nabla u|^{2} d \lambda_{2}\right) .
$$

The expressions on both sides of this estimate are conformally invariant. Thus, by an application of an appropriate Möbius transformation, we may assume that $z=0 \in E(0)$. Then $K(z)=K(0)=\mathcal{B}(0, \tanh (\delta / 2))$.

Let $\left.u\right|_{E(z)} \equiv a$. For $t \in[0, \tanh (\delta / 2)]$, consider the circles $\beta_{t}=\partial \mathcal{B}(0, t)$. For any such $t$ we have $\beta_{t} \cap E(0) \neq \varnothing$ because $\operatorname{diam}_{H} E(0) \geq \delta$. Moreover, we may assume that for almost all $t \in[0, \tanh (\delta / 2)]$ the function $u$ is absolutely continuous on $\beta_{t}$ and takes the value $a$ on this circle. Arguing as in the proof of Theorem 3.3 , we obtain the estimate

$$
2 \pi t a^{2} \leq 2 \int_{\beta_{t}} u^{2} d \mathcal{H}^{1}+8 \pi^{2} t^{2} \int_{\beta_{t}}|\nabla u|^{2} d \mathcal{H}^{1} .
$$

If $\zeta=t e^{i \theta} \in \beta_{t}, \theta \in[0,2 \pi]$, then $\left(\left.d \mathcal{H}^{1}\right|_{\beta_{t}}\right)(\zeta)=t d \theta, d A(\zeta)=\frac{4 t d t d \theta}{\left(1-t^{2}\right)^{2}} \asymp d \lambda_{2}(\zeta)$ on $K(0)$ (the constant of comparability is absolute: recall that it is assumed that $\delta \leq \frac{1}{3}$ ). Taking this into account and integrating (33) over $t \in[0, \tanh (\delta / 2)]$, we get (32) with some constant $\widetilde{C}_{2}$ depending only on $\delta$.

Now we sum up (32) over all $z \in X$ to obtain

$$
\sum_{j \in J} n_{j} a_{j}^{2} \leq \nu \widetilde{C}_{2} \cdot\left(\int_{\mathbb{D}} u^{2} d A+\int_{\mathbb{D}}|\nabla u|^{2} d \lambda_{2}\right),
$$

since the disks $K(z), z \in X$, have overlap multiplicity not exceeding $\nu$; on the left-hand side we have exactly this sum because $\operatorname{card}\left(E_{j} \cap X\right)=n_{j}$ for all $j \in J$. The assertion of Theorem 2.5 can be rewritten in the following way:

$$
\int_{\mathbb{D}} u^{2} d A \leq \mathrm{const} \cdot\|u\|_{W^{1,2}(\mathbb{D})}^{2}, \quad u \in \stackrel{\circ}{W}^{1,2}(\mathbb{D}),
$$

with an absolute constant on the right-hand side. This and previous inequalities imply the claim of the Lemma. 
Now we continue the proof of Theorem 5.4 Let us estimate $\sum_{j \in J_{1}^{\delta}} a_{j}^{2}$. In each set $B_{j}$, $j \in J_{1}^{\delta}$, we pick a point $\zeta_{j}$ and put $X:=\left\{\zeta_{j}\right\}_{j \in J_{1}^{\delta}}$. The set $X$ is uniformly locally finite, because a disk of the form $\mathcal{B}_{H}(z, 1)$ cannot intersect more than $N(\Omega)$ holes $B_{j}$. By the definition of the set $J_{1}^{\delta}$, if $j \in J_{1}^{\delta}$, then $\operatorname{diam}_{H}\left(B_{j}\right) \geq \delta$. Application of Lemma [5.6 allows us to conclude that

$$
\sum_{j \in J_{1}^{\delta}} a_{j}^{2} \leq C_{1} \cdot\|u\|_{W^{1,2}(\mathbb{D})}^{2},
$$

where $C_{1}<+\infty$ depends only on $N(\Omega)$ and $\delta$, i.e., only on constants occurring in the assumptions of the theorem.

Recall that, by the choice of the set of indices, if $j \notin J_{1}^{\delta}$, then $\operatorname{diam}_{H} B_{j}<\delta$.

Lemma 5.7. Let $B_{j}, B_{k}$ be two holes adjacent in the graph $g(\Omega, s)$, i.e., $\operatorname{Cap}_{2}\left(B_{j}, B_{k}\right) \geq s$. Suppose that $\operatorname{diam}_{H} B_{j}<\delta$ with the constant $\delta$ chosen at the beginning of the proof of Theorem [5.4] suppose also that $\operatorname{diam}_{H} B_{j} \leq \operatorname{diam}_{H} B_{k}$.

There exists a Borel set $A_{j k} \subset \mathbb{D}$ such that:

(1) if $u \in \mathcal{A d m}(\Omega)$, then $\left|\left(\left.u\right|_{B_{j}}\right)-\left(\left.u\right|_{B_{k}}\right)\right|^{2} \leq C_{2} \cdot \int_{A_{j k}}|\nabla u|^{2} d \lambda_{2}$;

(2) the inequalities $\operatorname{dist}_{H}\left(z, B_{j}\right) \leq C_{3}$ and $\operatorname{dist}_{H}\left(z, B_{k}\right) \leq C_{3}$ are fulfilled for any point $z \in A_{j k}$, and moreover, the sets $A_{j k} \cap B_{j}$ and $A_{j k} \cap B_{k}$ are nonempty and even infinite.

The constants $C_{2}, C_{3}<+\infty$ depend only on $\delta$ and $s$.

Proof. All the quantities occurring in the lemma are conformally invariant. Thus, by an application, if necessary, of a Möbius automorphism of the disk $\mathbb{D}$, we may assume that $0 \in B_{j}$. Since the Euclidean distance is not greater than the hyperbolic one, we have $\operatorname{diam} B_{j} \leq \delta$.

By the choice of $F$ made at the beginning of the proof of Theorem 5.4, we have $\operatorname{dist}\left(B_{j}, B_{k}\right) \leq F \cdot \operatorname{diam} B_{j}$. Thus, there exists $z_{1} \in B_{k}$ such that $\left|z_{1}\right| \leq(F+1) \cdot \operatorname{diam} B_{j}$. By the choice of $\delta$ we have $\left|z_{1}\right| \leq \frac{1}{3}$. Applying a rotation if necessary, we may assume that $z_{1} \in(0,1)$.

Put $\Lambda=\operatorname{diam} B_{j}$. Then $\operatorname{diam}_{H} B_{j} \geq \Lambda$. We show that diam $B_{k} \geq \frac{\Lambda}{4}$. Suppose the contrary. Then, since $z_{1} \in B_{k}$, and $\left|z_{1}\right| \leq(F+1) \Lambda$, we conclude that $B_{k} \subset \mathcal{B}(0,(F+2) \Lambda)$. In that disk, by the choice of $\delta$, the density of the metric $\frac{2|d z|}{1-|z|^{2}}$ with respect to the Euclidean metric does not exceed 4 . Then the estimate diam $B_{k}<\frac{\Lambda}{4}$ implies that $\operatorname{diam}_{H} B_{k}<\Lambda$. But diam $B_{k} \geq \operatorname{diam}_{H} B_{j} \geq \Lambda$, a contradiction. Hence, diam $B_{k} \geq \frac{\Lambda}{4}$.

For $t \in\left[0, \frac{\Lambda}{8}\right]$ we construct a contour $\beta_{t}$ formed by two circles $\partial \mathcal{B}(0, t)$ and $\partial \mathcal{B}\left(z_{1}, t\right)$ and also by two line segments $\left[0, z_{1}\right]+i t$ and $\left[0, z_{1}\right]-i t$. This contour is connected. Since $\operatorname{diam} B_{j}$, diam $B_{k} \geq \frac{\Lambda}{4}$, the sets $B_{j}$ and $B_{k}$ intersect $\beta_{t}$ for all $t<\frac{\Lambda}{8}$ (because $0 \in B_{j}$, $\left.z_{1} \in B_{k}\right)$.

Suppose $u \in \mathcal{A} d m(\Omega),\left.u\right|_{B_{j}}=a_{j},\left.u\right|_{B_{k}}=a_{k}$. We estimate $\left|a_{j}-a_{k}\right|$. Correcting, if necessary, the function $u$, we may assume that for almost every $t \in\left[0, \frac{\Lambda}{8}\right]$ the function $u$ is absolutely continuous on $\beta_{t}$ and takes values $a_{j}$ and $a_{k}$ on this contour. For all such $t$ we have

$$
\begin{aligned}
\left|a_{j}-a_{k}\right| \leq \int_{\beta_{t}}|\nabla u| d \mathcal{H}^{1} & \leq \sqrt{4 \pi t+2\left|z_{1}\right|} \cdot\left(\int_{\beta_{t}}|\nabla u|^{2} d \mathcal{H}^{1}\right)^{1 / 2} \\
& \leq \sqrt{\Lambda(\pi / 2+2 F+2)} \cdot\left(\int_{\beta_{t}}|\nabla u|^{2} d \mathcal{H}^{1}\right)^{1 / 2}
\end{aligned}
$$

(we have used the fact that the length of $\beta_{t}$ is $4 \pi t+2\left|z_{1}\right|$ ). 
Define the set $A_{j k}:=\bigcup_{t \in\left[0, \frac{\Lambda}{8}\right]} \beta_{t}$. Any point in $A_{j k}$ lies on $\beta_{t}$ for at most three values of $t$. Squaring (35) and integrating over $t \in\left[0, \frac{\Lambda}{8}\right]$ yields

$$
\frac{\Lambda\left|a_{j}-a_{k}\right|^{2}}{8} \leq 3 \Lambda(2 F+4) \cdot \int_{A_{j k}}|\nabla u|^{2} d \lambda_{2}
$$

which implies the first assertion of the lemma with $C_{2}=48(F+2)$, this factor depends only on $F$, that is only on $s$.

Now we check the second assertion. By construction, the distance from the origin to each of the point of the contour $\beta_{t}$ does not exceed $\Lambda\left(F+\frac{9}{8}\right)<\delta(F+2)$. Then, by the assumptions made on the choice of $\delta$, for any $z \in A_{j k}$ we have $|z|<\frac{1}{3}$; but also $0 \in B_{j}$, $z_{1} \in B_{k},\left|z_{1}\right|<\frac{1}{3}$. Therefore, $\operatorname{dist}_{H}\left(z, B_{j}\right) \leq C_{3}$, $\operatorname{dist}_{H}\left(z, B_{k}\right) \leq C_{3}$ with an absolute constant $C_{3}<+\infty$. Finally, the sets $A_{j k} \cap B_{j}, A_{j k} \cap B_{k}$ are infinite because $\beta_{t}$ intersects $B_{j}$ and $B_{k}$ for any $t<\frac{\Lambda}{8}$. The lemma is proved.

Now, for any pair of indices $j, k=1,2, \ldots$ such that $j \notin J_{1}^{\delta}$ or $k \notin J_{1}^{\delta}$, we construct sets $A_{j k}$ the existence of which was stated in Lemma 5.7 (Application of this lemma is justified by the definition of the set $J_{1}^{\delta}$.) The overlap multiplicity of such sets $A_{j k}$ does not exceed $N\left(\Omega, C_{3}\right)^{2}$, where $C_{3}$ is the constant from Lemma 5.7. Indeed, if $z \in A_{j k}$ then, by the second assertion of that lemma, $\operatorname{dist}_{H}\left(z, B_{j}\right)<C_{3}$ and $\operatorname{dist}_{H}\left(z, B_{k}\right)<C_{3}$. But the disk $\mathcal{B}_{H}\left(z, C_{3}\right)$ can intersect at most $N\left(\Omega, C_{3}\right)$ holes $B_{j}$. Therefore, the point $z$ cannot lie in more than $N\left(\Omega, C_{3}\right)^{2}$ sets of the form $A_{j k}$.

Now we estimate $\sum_{j \in J_{2}^{\delta}}\left(\left.u\right|_{B_{j}}\right)^{2}$ from above in terms of $\|u\|_{W^{1,2}(\mathbb{D})}^{2}$. If $j \in J_{2}^{\delta}$, then there exists $k=k(j) \in J_{1}^{\delta}$ such that the vertices $B_{j}$ and $B_{k(j)}$ are adjacent in the graph $g(\Omega, s)$. By the first assertion of Lemma 5.7 we have

$$
a_{j}^{2} \leq 2 a_{k(j)}^{2}+2 C_{2} \int_{A_{j k(j)}}|\nabla u|^{2} d \lambda_{2}
$$

For each $j \in J_{2}^{\delta}$, we mark a point $\zeta_{j}$ on the set $A_{j k(j)} \cap B_{k(j)}$ so that all the marked points are distinct (this can be done by the second assertion of Lemma 5.7); let $X$ denote the set of all marked points.

Let us check the uniform local finiteness of the set $X$. If some disk $\mathcal{B}_{H}\left(z_{0}, 1\right)$ contains a point $\zeta_{j}, j \in J_{2}^{\delta}$, then, by the second assertion of Lemma 5.7 $\mathcal{B}_{H}\left(z_{0}, 1+C_{3}\right) \cap B_{j} \neq \varnothing$. Therefore, $\mathcal{B}_{H}\left(z_{0}, 1\right)$ contains at most $N\left(\Omega, C_{3}+1\right)$ points $\zeta_{j}$, as claimed.

Let $k \in J_{1}^{\delta}$, and put $n_{k}:=\operatorname{card}\left(X \cap B_{k}\right)$ (this number is finite due to the uniform local finiteness property of $X$ and the compactness of $B_{k}$ ). Now we sum up the inequalities ( $\underline{36}$ ) over all $j \in J_{2}^{\delta}$ : we have

$$
\sum_{j \in J_{2}^{\delta}} a_{j}^{2} \leq 2 \sum_{k \in J_{1}^{\delta}} n_{k} a_{k}^{2}+2 C_{2} \sum_{j \in J_{2}^{\delta}} \int_{A_{j k(j)}}|\nabla u|^{2} d \lambda_{2} \leq 2 \sum_{k \in J_{1}^{\delta}} n_{k} a_{k}^{2}+2 C_{2} N\left(\Omega, C_{3}\right)^{2} \cdot\|u\|_{W^{1,2}(\mathbb{D})}^{2}
$$

because the overlap multiplicity of the sets $A_{j k(j)}, j \in J_{2}^{\delta}$, is does not exceed $N\left(\Omega, C_{3}\right)^{2}$. By Lemma 5.6 applied to the family $\left\{B_{k}\right\}_{k \in J_{1}^{\delta}}$ and the set $X$, we have an estimate of the form $\sum_{k \in J_{1}^{\delta}} n_{k} a_{k}^{2} \leq C_{4} \cdot\|u\|_{W^{1,2}(\mathbb{D})}^{2}$, where $C_{4}<+\infty$ depends only on $s$ and $N(\Omega)$. So, we have

$$
\sum_{j \in J_{2}^{\delta}} a_{j}^{2} \leq C_{5} \cdot\|u\|_{W^{1,2}(\mathbb{D})}^{2}
$$

with a constant $C_{5}<+\infty$, depending only on constants from the hypothesis of the theorem but not on $u \in \mathcal{A} d m(\Omega)$. (Recall that $s$ depends only on $S$.) 
It remains to estimate $\sum_{j \notin J_{1}^{\delta} \cup J_{2}^{\delta}} a_{j}^{2}$. The degree of any vertex $B_{j}, j \notin J_{1}^{\delta}$, in the graph $g(\Omega, s)$ is at most $N(\Omega)$. Indeed, consider such a vertex $B_{j}$. By applying a Möbius automorphism of the disk $\mathbb{D}$, we may assume that $0 \in B_{j}$. By the definition of the set $J_{1}^{\delta}$, we have $\operatorname{diam}_{H}\left(B_{j}\right) \leq \delta$ if $j \notin J_{1}^{\delta}$; then $\operatorname{diam} B_{j} \leq \delta$. By the choice of $\delta$, if $B_{k}$ is adjacent to $B_{j}$ in the graph $g(\Omega, s)$, then $B_{k} \cap \mathcal{B}\left(0, \frac{1}{3}\right) \neq \varnothing$. Since the number of such sets does not exceed $N(\Omega)$, the degree of the vertex $B_{j}$ is at most $N(\Omega)$.

Note that the degree of a vertex $B_{j}$ can be large if $\operatorname{diam}_{H}\left(B_{j}\right)$ is large, which may occur for $j \in J_{1}^{\delta}$. That is why we do estimates for $a_{j}$ with $j \in J_{2}^{\delta}$ separately. If the condition $\sup _{j \in \mathbb{N}} \operatorname{diam}_{H}\left(B_{j}\right)<+\infty$ is added to the hypothesis of the theorem, then we will be able to avoid application of Lemma 5.6 and estimate $a_{j}$ for all $j \notin J_{1}^{\delta}$ in the same manner.

For each $B_{j_{0}}$ with $j_{0} \notin J_{1}^{\delta} \cup J_{2}^{\delta}$, in the graph $g(\Omega, s)$ there exists a path of length not exceeding $M$ (where $M \in \mathbb{N}$ is the constant from the hypothesis of theorem) that connects $B_{j_{0}}$ with one of the vertices $B_{l}, l \in J_{2}^{\delta}$. Indeed, there exists a path with at most $M$ edges connecting $B_{j_{0}}$ and $\mathbb{D}^{(c)}$. The prenultimate vertex in this path lies in $J_{1}^{\delta}$ (indeed, if $\operatorname{Cap}_{2}\left(B_{k}, \mathbb{D}^{(c)}\right) \geq s$, then, by the choice of $\delta$, we have $\operatorname{diam}_{H}\left(B_{k}\right) \geq \delta$, and therefore $k \in J_{1}^{\delta}$ ). Hence, this path contains some vertices with indices in $J_{1}^{\delta}$ and, therefore, some vertices with indices in $J_{2}^{\delta}$. Taking the last of them as $B_{l}$, we obtain a path $\left(B_{j_{0}}, B_{j_{1}}, \ldots, B_{j_{m}}\right)$ in the graph $g(\Omega, s)$ such that $j_{1}, j_{2}, \ldots, j_{m} \notin J_{1}^{\delta}$ but $j_{m} \in J_{2}^{\delta}$. At the same time, $m \leq M$. We denote by $\mathcal{M}$ the set of all such paths; each of them corresponds to some set $B_{j_{0}}, j_{0} \notin J_{1}^{\delta} \cup J_{2}^{\delta}$.

Since $j_{0}, j_{1}, \ldots, j_{m} \notin J_{1}^{\delta}$, the sets $A_{j_{k} j_{k+1}}, k=0,1, \ldots, m-1$, are well defined. By the construction in Lemma 5.7, for all such $k$ we have

$$
a_{j_{k}}^{2} \leq 2 a_{j_{k+1}}^{2}+2 C_{2} \cdot \int_{A_{j_{k} j_{k+1}}}|\nabla u|^{2} d \lambda_{2}
$$

Applying this for $k=0,1, \ldots, m-1$, we obtain an estimate of the form

$$
a_{j_{0}}^{2} \leq C_{6} \cdot\left(a_{j_{m}}^{2}+\sum_{k=0}^{m-1} \int_{A_{j_{k} j_{k+1}}}|\nabla u|^{2} d \lambda_{2}\right)
$$

where $C_{6}<+\infty$ depends only on $m$ and $C_{1}$. Since $m \leq M$, the constant $C_{6}$ can be bounded from above in terms of the constants from the hypothesis of the theorem.

All paths belonging to $\mathcal{M}$ have lengths at most $M$ and go by vertices $B_{j}, j \notin J_{1}^{\delta}$. The degrees of such vertices do not exceed $N(\Omega)$. Thus, there exists $L \in \mathbb{N}$ such that each edge in the graph $g(\Omega, s)$ belongs to at most $L$ paths of class $\mathcal{M}$, and moreover, any vertex $B_{j}$ with $j \in J_{2}^{\delta}$ is the endpoint of at most $L$ paths of class $\mathcal{M}$. We sum up estimates (38) over all $j_{0} \notin J_{1}^{\delta} \cup J_{2}^{\delta}$; since the sets $A_{j k}$ have overlap multiplicity not exceeding $N\left(\Omega, C_{3}\right)^{2}$, we can conclude that

$$
\sum_{j \notin J_{1}^{\delta} \cup J_{2}^{\delta}} a_{j}^{2} \leq C_{6} L \cdot \sum_{k \in J_{2}^{\delta}} a_{k}^{2}+C_{6} L \cdot N\left(\Omega, C_{3}\right)^{2} \cdot \int_{\mathbb{D}}|\nabla u|^{2} d \lambda_{2} .
$$

Since the quantity $\sum_{k \in J_{2}^{\delta}} a_{k}^{2}$ has already been estimated (see inequality (37)), we obtain an estimate of the form

$$
\sum_{j \notin J_{1}^{\delta} \cup J_{2}^{\delta}} a_{j}^{2} \leq C_{7} \cdot\|u\|_{W^{1,2}(\mathbb{D})}^{2},
$$


where $C_{7}<+\infty$ depends only on constants from the hypothesis of the theorem. Combining estimates (34), (37), and (39), we see that if $u \in \mathcal{A} d m(\Omega)$, then

$$
\sum_{j=1}^{\infty} a_{j}^{2} \leq C_{8} \cdot\|u\|_{W^{1,2}(\mathbb{D})}^{2}
$$

with some constant $C_{8}<+\infty$ depending only on constants from the hypothesis of the theorem. The proof is finished.

Remark. The interpolation property may fail if we erase some holes in the domain $\Omega$. Consider, for example, the domain $\widetilde{\Omega}_{\delta}$ with two holes that we constructed in Example 3.3 in Subsection 3.3. The quantities $C_{I}\left(\widetilde{\Omega}_{\delta}\right)$ are bounded uniformly. If we erase the greatest hole $\widetilde{B}_{1}$ in this domain, then the interpolation constant of the domain $\mathbb{D} \backslash \widetilde{B}_{2}^{\delta}$ will become large if $\delta$ is small. (This follows from Theorem 3.4 and the smallness of the quantity $\operatorname{diam}_{H}\left(\widetilde{B}_{2}^{\delta}\right)$.) Performing a construction that employs conformal shifts like in Example 3.3 in Subsection 3.3. one can obtain a domain $\Omega$ possessing the interpolation property but such that this property will fail after erasing a countable family of holes in $\Omega$.

In terms of the graph $G(\Omega, S)$, this nonmonotonicity with respect to domains can be understood as follows: the connectedness of a graph may fail if we remove some of its vertices. If we erase the hole $B_{j}$ in the domain in Figure 11, then the vertex $B_{k}$ will become not connected with $\mathbb{D}^{(c)}$ in the graph $G\left(\Omega \cup B_{j}, S\right)$ if, of course, we do not enlarge $S$.

The nonmonotonicity mentioned above can also be explained geometrically. Let $\Omega_{1}$ be a domain obtained by erasing some holes $B_{j}, j \in J$, in a domain $\Omega$. The constant $C_{I}\left(\Omega_{1}\right)$ can be large whereas $C_{I}(\Omega)$ is small under the following circumstances. Suppose that a form $\omega \in L_{c}^{2,1}\left(\Omega_{1}\right)$ has given periods in $\Omega_{1}$ and minimizes the integral $\int_{\Omega_{1}}|\omega|^{2} d \lambda_{2}$ under this condition. It may happen that the main mass of this integral falls into the union of the holes erased, and then the integral $\int_{\Omega}|\omega|^{2} d \lambda_{2}$ may turn out to be not large.

On the contrary, the Bessel property is preserved if we erase some holes in $\Omega$. The Bessel constant will not increase after this removal. This can be seen immediately from the definition of the Bessel property.

5.3. Complete interpolation criterion. Theorem 4.2, Proposition 2.20 , Theorem 5.4 Theorem [5.1, and Theorem [5.5] which have all already been proved, immediately imply the following statement.

Theorem 5.8 (complete interpolation criterion). Let $\Omega$ be a regular domain obtained by removing from the disk $\mathbb{D}$ connected holes $B_{j}, j=1,2, \ldots$, that have smooth boundaries and do not accumulate to inner points of $\mathbb{D}$. The domain $\Omega$ possesses the complete interpolation property (that is, the range of the operator Per determined by $\Omega$ coincides with $\ell^{2}$ ) if and only the following conditions are satisfied.

(1) The family of holes $\left\{B_{j}\right\}_{j=1}^{\infty}$ is uniformly locally finite, that is, any disk of radius 1 in the hyperbolic metric intersects at most $N=N(\Omega)<+\infty$ holes $B_{j}$.

(2) $\sup _{j \in \mathbb{N}} \operatorname{diam}_{H}\left(B_{j}\right)<+\infty$, where $\operatorname{diam}_{H}$ is hyperbolic diameter.

(3) The holes $B_{j}$ are $\varepsilon$-weakly separated with some $\varepsilon>0$, i.e.,

$$
\operatorname{dist}\left(B_{j}, B_{k}\right) \geq \varepsilon \cdot \min \left\{\operatorname{diam} B_{j}, \operatorname{diam} B_{k}\right\}
$$

for all $j, k=1,2, \ldots, j \neq k$.

(4) For some $S<+\infty$ the graph $G(\Omega, S)$ defined at the beginning of Subsection 5.2 is connected and its diameter $M$ is finite. 
Moreover, if $\Omega$ has the complete interpolation property, then the quantities $M, S, N(\Omega)$, and $\sup _{j \in \mathbb{N}} \operatorname{diam}_{H}\left(B_{j}\right)$ can be estimated from above, whereas $\varepsilon$ can be estimated from below, by constants depending only on $C_{I}(\Omega)$ and $C_{B}(\Omega)$.

Vice versa, if conditions $1-4$ are satisfied, then the constants $C_{I}(\Omega)$ and $C_{B}(\Omega)$ can be estimated from above only in terms of $\varepsilon, M, S, N(\Omega)$, and $\sup _{j \in \mathbb{N}} \operatorname{diam}_{H}\left(B_{j}\right)$.

Remark. The author suspects that there exist abstract countable graphs whose connectedness is difficult to check mathematically or algorithmically. In our problem the situation simplifies, because in Theorems 5.4 and 5.5 we have the condition that the diameter of $G(\Omega, S)$ is finite. If also $\sup _{j \in \mathbb{N}} \operatorname{diam}_{H}\left(B_{j}\right)<+\infty$ and the holes in $\Omega$ are uniformly locally finite, then the degrees of the vertices $B_{j}$ in $G(\Omega, S)$ can be estimated from above. This allows us to check (at least, algorithmically) the inequality $\operatorname{dist}_{G(\Omega, S)}\left(B_{j}, \mathbb{D}^{(c)}\right) \leq M$ for given $S, M$, and $j$, of course, if we know everything about the metric of holes.

\section{$\S 6$. Comments}

6.1. The Blaschke condition for regular domains. As a side result of the proofs of Theorem 4.2 on uniform local finiteness and Theorem 3.4, we have obtained the estimate $\sum_{j=1}^{\infty} \operatorname{dist}\left(B_{j}, \partial \mathbb{D}\right)^{2}<+\infty$. It is natural to ask, for what exponents $\alpha \in \mathbb{R}$ the series $\sum_{j=1}^{\infty} \operatorname{dist}\left(B_{j}, \partial \mathbb{D}\right)^{\alpha}$ will necessarily converge if the domain $\Omega$ possesses the complete interpolation property. The answer can easily be obtained only from the uniform local finiteness property: for $\alpha>1$.

Indeed, suppose that $\Omega$ has the uniform local finiteness property (by Theorem 4.2 , this is the case if $\Omega$ possesses the complete interpolation property). In each hole $B_{j}$, mark a point $z_{j}$ closest to the circle $\partial \mathbb{D}$. For $n=1,2, \ldots$ and $k=0,1,2, \ldots, 2^{n}-1$, consider the dyadic sectors

$$
Q_{n, k}=\left\{r e^{i \theta}: r \in\left(1-2^{-n+1}, 1-2^{-n}\right], \theta \in\left[2 \pi k \cdot 2^{-n}, 2 \pi(k+1) \cdot 2^{-n}\right)\right\} .
$$

We have $\operatorname{diam}_{H} Q_{n, k} \leq 20$. Therefore, each $Q_{n, k}$ contains at most $N(\Omega, 20)$ marked points $z_{j}$. If $z_{j} \in Q_{n, k}$, then $1-\left|z_{j}\right| \leq 2^{-n+1}$. Hence,

$$
\begin{aligned}
\sum_{j=1}^{\infty} \operatorname{dist}\left(B_{j}, \partial \mathbb{D}\right)^{\alpha} & \leq \sum_{j=1}^{\infty}\left(1-\left|z_{j}\right|\right)^{\alpha} \leq \sum_{n=1}^{\infty} \sum_{k=0}^{2^{n}-1} \sum_{j: z_{j} \in Q_{n, k}}\left(1-\left|z_{j}\right|\right)^{\alpha} \\
& \leq \sum_{n=1}^{\infty} 2^{n} \cdot N(\Omega, 20) \cdot 2^{-\alpha(n-1)}<+\infty
\end{aligned}
$$

whenever $\alpha>1$.

The series $\sum_{j=1}^{\infty} \operatorname{dist}\left(B_{j}, \partial \mathbb{D}\right)$ may fail to converge when the domain $\Omega$ has the complete interpolation property. Indeed, in any dyadic sector $Q_{n, k}(n=1,2, \ldots ; k=$ $\left.0,1,2, \ldots, 2^{n}-1\right)$, place a round hole $B_{n, k}$ centered at $r e^{i \theta}$, where $r=1-3 \cdot 2^{-n-1}$, $\theta=2 \pi\left(k+\frac{1}{2}\right) \cdot 2^{-n}$, and set the radius of the hole $B_{n, k}$ to be $2^{-n} / 100$. It is easily seen that the holes $B_{n, k}$ defined in this way are strongly separated and their hyperbolic diameters are bounded above and separated away from zero. Then we may apply Theorem 3.7. and the domain $\Omega$ equal to the disk $\mathbb{D}$ without the holes $B_{n, k}$ has the complete interpolation property. At the same time, $\operatorname{dist}\left(B_{n, k}, \partial \mathbb{D}\right) \geq 2^{-n}$, whence $\sum_{n=1}^{\infty} \sum_{k=0}^{2^{n}-1} \operatorname{dist}\left(B_{n, k}, \partial \mathbb{D}\right) \geq \sum_{n=1}^{\infty} 2^{n} \cdot 2^{-n}=+\infty$.

6.2. Domains with nonsmooth holes. Our investigation of the problem on interpolation by periods is, in general, based on reproducing kernels. In this way we repeatedly (in the proofs of Theorem 2.4 and Proposition 2.10) needed the smoothness of these kernels up to the boundaries of the holes in the domain. We would like to consider the 
problem on interpolation by periods also in domains with nonsmooth holes 2 . Here we shall not rely on reproducing kernels. Instead, we shall pass to the limit, approximating the nonsmooth holes by smooth ones. The statement of our problem does not involve the smoothness of holes; in the criteria we obtained we sometimes used capacity (which was defined by a way appropriate only for sufficiently "fat" sets), but we can avoid this and rewrite the conditions involved in terms of metrics. We need check that our problem as well as the answers obtained admit a limit passage if we approximate a domain with arbitrary holes by domains with locally smooth boundaries in an appropriate way. The idea of such an approximation is rather clear; we will meet some minor technical difficulties only in Lemma 6.1 and Proposition 6.7.

So, let $B_{1}, B_{2}, \ldots$ be connected disjoint compact sets (continua) in the disk $\mathbb{D}$. We shall suppose that each $B_{j}$ does not separate the plane $\mathbb{C}$ and consists of more than one point; and also that the sets $B_{j}, j=1,2, \ldots$, accumulate only to the boundary of the disk $\mathbb{D}$. Put $\Omega:=\mathbb{D} \backslash \bigcup_{j=1}^{\infty} B_{j}$. As before, we say that the sets $B_{j}, j=1,2, \ldots$, are holes in $\Omega$, and such a domain $\Omega$ itself will be called a domain with arbitrary holes.

The space $L_{c}^{2,1}(\Omega)$ is defined in the same way as in the case of domains with smooth holes. It is easy to show that for each $j=1,2, \ldots$ there exists a curve $\gamma_{j} \subset \Omega$ winding around $B_{j}$ once in the positive direction and not winding around the other holes in $\Omega$. The functional $\operatorname{Per}_{j}^{(\Omega)}(\omega)=\int_{\gamma_{j}} \omega, \omega \in L_{c}^{2,1}(\Omega)$ is well defined and continuous on $L_{c}^{2,1}(\Omega)$. The operator $\operatorname{Per}^{(\Omega)}: L_{c}^{2,1}(\Omega) \rightarrow \ell^{2}$ is defined in the same way as in the case of smooth holes. The Bessel, weak Bessel, interpolation, and complete interpolation properties and also the constants $C_{B}(\Omega), \widetilde{C}_{B}(\Omega)$, and $C_{I}(\Omega)$ for the domains of the above-mentioned type are introduced like in Definition 1.2 . Proposition 1.3 stays true for domains with arbitrary holes. Corollary 2.11 on monotonicity with respect to domains also remains true for such domains, because this can be proved directly via forms and with no use of reproducing kernels.

Let $\Omega$ be a domain with arbitrary holes, and let $B_{1}, B_{2}, \ldots$ be the bounded connected components of $\Omega^{(c)}$. Let $\Omega_{m}, m=1,2, \ldots$, be a sequence of regular domains, and let $B_{1, m}, B_{2, m}, \ldots$ be holes in $\Omega_{m}$. We say that the sequence $\left\{\Omega_{m}\right\}_{m=1}^{\infty}$ increases to the domain $\Omega$ nicely if for each $j=1,2, \ldots$ the sets $B_{j, m}$ decrease to the hole $B_{j}$ as $m \rightarrow+\infty$.

Lemma 6.1. Let $\Omega$ be a domain with arbitrary holes. There exists a sequence of domains $\Omega_{m}, m=1,2, \ldots$, nicely increasing to $\Omega$ and such that

(1) the quantities $C_{B}\left(\Omega_{m}\right)$ decrease to $C_{B}(\Omega)$;

(2) the quantities $\widetilde{C}_{B}\left(\Omega_{m}\right)$ decrease to $\widetilde{C}_{B}(\Omega)$;

(3) the quantities $C_{I}\left(\Omega_{m}\right)$ increase to $C_{I}(\Omega)$.

The proof is given in the Appendix. This is the only point in our work when we make an essential use of the theory of conformal mappings. The third assertion of Lemma 6.1 is true for any nicely increasing sequence of domains. The first and second limit passages may fail in the case of an unsuccessful choice of such a sequence. It suffices to take the domain with conformal symmetry constructed in Example 3.3 in Subsection 3.3 and replace the round holes in this domain by disks concentric with them and of small hyperbolic diameters one-by-one.

Corollary 6.2. If a domain $\Omega$ with arbitrary holes possesses the weak Bessel property, then it has the Bessel property, moreover, $C_{B}(\Omega) \leq \sqrt{2} \widetilde{C}_{B}(\Omega)$.

\footnotetext{
${ }^{2}$ The appropriateness of consideration of such domains, in particular, domains with line slits, was pointed out by E. L. Korotyaev.
} 
Proof. It suffices to apply Theorem 2.16 to the sequence of domains $\Omega_{m}$ constructed in Lemma 6.1 and pass to the limit in the estimate $C_{B}\left(\Omega_{m}\right) \leq \sqrt{2} \widetilde{C}_{B}\left(\Omega_{m}\right)$.

Remark. If we enlarge any of the domains constructed in Lemma 6.1 so that the resulting sequence will still consist of regular domains and will increase to $\Omega$ monotonically, then the assertion of Lemma 6.1 will still be true. This follows from the monotonicity of all the constants in that lemma.

Definition 4.1 of uniform local finiteness property and the constants $N(\Omega), N(\Omega, d)$ remains the same for domains with arbitrary holes.

Proposition 6.3. If the domain $\Omega$ with arbitrary holes possesses the uniform local finiteness property, then the sequence of domains $\Omega_{m}, m=1,2, \ldots$, constructed in Lemma 6.1 can also be subject to the uniform local finiteness condition in order to have $N\left(\Omega_{m}, d\right) \leq N(\Omega, d+1)$ for all $m=1,2, \ldots$ and $d \geq 0$.

Proof. Indeed, for this it suffices to act so that for all $m=1,2, \ldots$ and $j=1,2, \ldots$ the set $B_{j, m}$ lies in the 1-hyperbolic neighborhood of the set $B_{j}$. But this is easy to achieve by shrinking the holes in the domains $\Omega_{m}$, preserving the regularity of these domains and the monotonicity of the sequence formed by them.

Proposition 6.4. If a domain $\Omega$ with arbitrary holes possesses the complete interpolation property, then it has the uniform local finiteness property. Moreover, $N(\Omega)$ can be estimated from above in terms of $C_{I}(\Omega)$ and $C_{B}(\Omega)$.

Proof. If $\Omega_{m}, m=1,2, \ldots$, is the sequence of domains constructed in Lemma 6.1, then, by the estimates in that lemma, there exists at least one $m_{0}=1,2, \ldots$ such that the domain $\Omega_{m_{0}}$ has the complete interpolation property with $C_{I}\left(\Omega_{m_{0}}\right) \leq C_{I}(\Omega), C_{B}\left(\Omega_{m_{0}}\right) \leq$ $2 C_{B}(\Omega)$. By Theorem 4.2, $N\left(\Omega_{m_{0}}\right)$ is finite and can be estimated in terms of $C_{I}(\Omega)$ and $C_{B}(\Omega)$. But so does the quantity $N(\Omega)$ because it does not exceed $N\left(\Omega_{m_{0}}\right)$.

Definition 2.18 of the $\varepsilon$-weak separation of holes remains the same for domains with arbitrary holes.

Proposition 6.5. If a domain $\Omega$ with arbitrary holes possesses the Bessel property, then the holes in this domain are $\varepsilon$-weakly separated with some $\varepsilon>0$ depending only on $C_{B}(\Omega)$.

Proof. The sequence of domains $\Omega_{m}, m=1,2, \ldots$, constructed in Lemma 6.1, by the estimates in that lemma, contains a domain $\Omega_{m_{0}}$ with $C_{B}\left(\Omega_{m_{0}}\right)<2 C_{B}(\Omega)$. By Proposition 2.20 , the holes in $\Omega_{m_{0}}$ are $\varepsilon$-weakly separated with some $\varepsilon>0$ depending only on $C_{B}(\Omega)$. But then the same is true also for $\Omega$.

Proposition 6.6. If a domain $\Omega$ with arbitrary holes possesses the uniform local finiteness property and if the holes in this domain are $\varepsilon$-weakly separated with $\varepsilon>0$ and also $\sup _{j \in \mathbb{N}} \operatorname{diam}_{H}\left(B_{j}\right)<+\infty$, then $\Omega$ has the Bessel property. Moreover, the constant $C_{B}(\Omega)$ can be estimated from above in terms of $\varepsilon, \sup _{j \in \mathbb{N}} \operatorname{diam}_{H}\left(B_{j}\right)$, and $N(\Omega)$.

The proof is given in the Appendix.

For $S \in(0,+\infty)$ and for a domain $\Omega$ with arbitrary holes, the graph $G(\Omega, S)$ is defined in the same way as at the beginning of Subsection 5.2. This definition involves only metric characteristics of holes in the domain, but not capacities.

Suppose that a sequence of domains $\Omega_{m}, m=1,2, \ldots$, increases nicely to a domain $\Omega$, and let $B_{1, m}, B_{2, m}, \ldots$ be holes in $\Omega_{m}$. For fixed $j_{0}=1,2, \ldots$, let us identify the corresponding vertices $B_{j_{0}, m}$ in all graphs of the form $G\left(\Omega_{m}, S\right)$ with different $S \in$ $(0,+\infty)$ and $m=1,2, \ldots$. Moreover, we identify the vertices $\mathbb{D}^{(c)}$ in all graphs $G\left(\Omega_{m}, S\right)$. 
Proposition 6.7. Let $\Omega$ be a domain with arbitrary holes, and let $N(\Omega)<+\infty$. If $\Omega$ possesses the interpolation property, then there exists $S<+\infty$ and $M \in \mathbb{N}$ such that the graph $G(\Omega, S)$ is connected and $\operatorname{dist}_{G(\Omega, S)}\left(B_{j}, \mathbb{D}^{(c)}\right) \leq M$ for any hole $B_{j}$.

The numbers $S$ and $M$ can be estimated from above in terms of $C_{I}(\Omega)$ and $N(\Omega)$.

The proof is given in the Appendix. Here the limit passage in the sequence of graphs $G\left(\Omega_{m}, S\right)$ requires a certain caution because the edge-wise limit of a sequence of connected graphs may turn out to be nonconnected if the degrees of vertices of these graphs may be infinite.

Proposition 6.8. Let $\Omega$ be a domain with arbitrary holes, and let $N(\Omega)<+\infty$. If, for some $S<+\infty$, the graph $G(\Omega, S)$ is connected and its diameter does not exceed some number $M \in \mathbb{N}$, then $\Omega$ has the interpolation property. Moreover, the interpolation constant $C_{I}(\Omega)$ can be estimated from above in terms of $N(\Omega), S$, and $M$.

Proof. Let $\Omega_{m}, m=1,2, \ldots$, be the sequence of domains constructed in Proposition 6.3. We identify the vertices of the graphs $G\left(\Omega_{m}, S\right)$ with those of $G(\Omega, S)$ as before Proposition 6.7. By the definition of these graphs, any edge from $G(\Omega, S)$ is also present in the graph $G\left(\Omega_{m}, S\right)$. Therefore, the graphs $G\left(\Omega_{m}, S\right)$ are connected for all $m=1,2, \ldots$ and their diameters do not exceed $M$. Moreover, $N\left(\Omega_{m}\right) \leq N(\Omega, 2)$ by Proposition 6.3. Hence, the constants $C_{I}\left(\Omega_{m}\right)$ are finite and admit a simultaneous upper estimate in terms of the constants from the hypothesis. But $C_{I}\left(\Omega_{m}\right) \stackrel{m \rightarrow \infty}{\longrightarrow} C_{I}(\Omega)$, so that the constant $C_{I}(\Omega)$ is also finite and admits the same estimate.

The results proved in this subsection immediately imply the next claim.

Corollary 6.9. The assertion of Theorem 5.8 stays true for domains with arbitrary holes.

\subsection{Some open questions.}

1. Higher dimensions. A straightforward generalization of our results to the case of higher dimensions seems to be difficult. Suppose that we study the question on interpolation by periods for differential forms of degree $k \in \mathbb{N}$ integrable with an exponent $p \in[1,+\infty]$ over an open set in $\mathbb{R}^{n}, n=2,3, \ldots$ (or an $n$-dimensional Riemann manifold). In this case it is natural to treat the period operator as an operator with range in $\ell^{p}$. It is convenient to have the scale-invariance of such a problem, the most of estimates for capacities are based on this fact. This invariance will be the case if $k p=n$.

On the other hand, Theorem 2.4 on the explicit form of the period reproducing kernels will be true in the same form if $k=n-1$; in this case duality converts a problem on forms to a problem on scalar functions. To have scale-invariance, we then have to take $p=n /(n-1)$. This particular case seems to be degenerate to the author's taste. Nevertheless, even in this situation it is impossible to translate the arguments leading to the solution of the problem for $n=2$ to a higher dimension. This is because the period reproducing kernels will still be gradients of harmonic functions. Writing the Riesz basis conditions for linear combinations of such kernels leads us to estimates of harmonic gradients in $L^{n}$-norm (because the exponent $n$ is conjugate to $p=n /(n-1)$ ). The passage from the Riesz basis conditions to those in Proposition 2.10 was possible due to the fact that harmonic functions minimize the $L^{2}$-norm of the gradient under fixed Dirichlet boundary data. But this passage will no longer be possible if we work with estimates of $L^{n}$-norms of such gradients when $n>2$. Therefore, even in the case where $k=n-1, p=n /(n-1)$, our arguments cannot be generalized directly to higher dimensions.

2. Riemann surfaces. The author thinks that results of our study can be generalized to the case of Riemann surfaces. Then we should give up the simple definition of $\Omega$ as 
a subset in $\mathbb{C}$, under which we were easily able to formulate metric conditions on the holes $B_{j}$. The author suspects that the problem can also be solved on Riemann surfaces, and that extremal lengths should stay instead of metrics.

3. Normed interpolation. Yet another generalization of our problem is the investigation of the normed period operator

$$
\omega \mapsto\left\{\operatorname{Per}_{j}(\omega) /\left\|\operatorname{Per}_{j}\right\|_{\left(L_{c}^{2,1}(\Omega)\right)^{*}}\right\}_{j=1}^{\infty}, \quad \omega \in L_{c}^{2,1}(\Omega) .
$$

Namely, the problem on interpolation by normalized periods is to clarify when such an operator is bounded and has bounded right inverse as an operator from $L_{c}^{2,1}(\Omega)$ to $\ell^{2}$. This problem differs from the studied problem on unnormalized interpolation by periods by the fact that in the case of unnormalized interpolation we assume that the norms $\left\|\operatorname{Per}_{j}\right\|_{\left(L_{c}^{2,1}(\Omega)\right)^{*}}, j=1,2, \ldots$, are separated from zero and bounded from above. The last condition (the weak Bessel property) gives us a lot of useful information on the geometry of $\Omega$.

The author has not paid any serious attention to the above-mentioned problem on interpolation by normalized periods. Nevertheless, we point out a case in which such a statement may happen to be relevant. Consider a nested sequence of domains $\Omega_{1} \supset$ $\Omega_{2} \supset \ldots$ convergent to the Sierpiński triangle $K$. Should the constants $C_{B}\left(\Omega_{j}\right)$ and $C_{I}\left(\Omega_{j}\right)$ stay bounded as $j \rightarrow \infty$, we would be able to pass to the limit over the domains, getting a good Hilbert (co)homology calculus on $K$. But, by Theorem 4.2 on the uniform local finiteness, the Bessel or interpolation constants of domains approximating $K$ should increase infinitely when domains tend to $K$, because the family of holes in $K$ is not uniformly locally finite. (Under a natural choice of the domains $\Omega_{j}$ we have $C_{B}\left(\Omega_{j}\right) \rightarrow \infty$.) Therefore, Theorem 4.2 on uniform local finiteness, which easily allowed us to describe the complete interpolation property of a regular domain, is also a negative result, which makes it impossible to pass to the limit over domains with locally smooth boundaries to domains with more complicated, that is, fractal configurations of holes. It may occur that such a limit passage will be possible exactly in the problem on normalized interpolation by periods. For the Sierpiński triangle, it seems to be possible to make direct calculations relying on the rich resource of its symmetries and in terms of only intrinsic geometry of this set.

4. Integral Riesz bases in Hilbert homologies. Finally, we state another generalization of the problem on interpolation by periods - the problem about an integral Riesz basis in Hilbert homologies.

Let $\Omega$ be a countably connected domain in $\mathbb{C}$. The spaces $H_{L^{2}}^{1}(\Omega)$ and $H_{1, L^{2}}(\Omega)$ of Hilbert cohomologies and homologies in $\Omega$ are defined as in the remark at the end of Subsection 2.3. These definitions make sense in the case where $\Omega$ is a countably connected Riemann surface.

The space $H_{1, L^{2}}(\Omega)$ is Hilbert and, of course, there are orthonormal bases and Riesz bases in this space. But from a geometrical viewpoint, the fact of their presence is too abstract. The question is if there are "geometrically natural" Riesz bases in $H_{1, L^{2}}(\Omega)$. For us, countable systems of integral homologies in $\Omega$ seem to be natural ones. The integral $L^{2}$-homologies in $\Omega$ can be defined as the functionals on $H_{L^{2}}^{1}(\Omega)$ that can be represented as $\omega \mapsto \int_{\beta} \omega, \omega \in H_{L^{2}}^{1}(\Omega)$, where $\beta$ is some smooth closed curve in $\Omega$. The set $\Omega$ is assumed to be connected, so that such homologies form an additive subgroup in $H_{1, L^{2}}(\Omega)$.

Consider the following property of a domain or Riemann surface $\Omega$ :

In the space of Hilbert homologies $H_{1, L^{2}}(\Omega)$, there exists a Riesz basis consisting of integral homologies. 
Proposition 1.3 implies that property $(\dagger)$ is preserved under application to $\Omega$ of a quasiconformal diffeomorphism. Note that the examples of inverse domains (Examples 3.3 and 3.3 in Subsection 3.3) show that even one inversion can throw our problem out of the class of problems on interpolation by periods around holes. That is, if we regard this problem as a conformally invariant one, then its statement is attached to a conformal implementation of $\Omega$ as a subset of the plane. Property $(\dagger)$ is free of such disadvantage, because there is no preferred homology system in that property. In other words, property $(\dagger)$ is geometrically invariant.

Now we translate property $(\dagger)$ into analytical language. Let $\Omega$ be a regular domain (the class of such domains was introduced in Subsection 1.1). Let, as usual, closed curves $\gamma_{j} \subset \Omega$ be winding around the corresponding holes $B_{j}, j=1,2, \ldots$ We identify these curves with homologies from $H_{1, L^{2}}(\Omega)$ given by them (one may also work with the kernels $\kappa_{j}$ reproducing the periods along the curves $\gamma_{j}$ ). Property $(\dagger)$ of the domain $\Omega$ is equivalent to the following one: there exists a Riesz basis in $H_{1, L^{2}}(\Omega)$ whose elements are finite integral linear combinations of the homologies $\gamma_{j}, j=1,2, \ldots$.

Let $H$ be an abstract Hilbert space and $\left\{x_{j}\right\}_{j=1}^{\infty}$ a countable system of vectors in $H$. Consider the following property of this system:

There exists a Riesz basis in $H$ whose elements are integral linear combinations of the vectors $x_{j}, j=1,2, \ldots$.

There are other properties similar to $(\ddagger)$ : one may require that the elements of the system $\left\{x_{j}\right\}_{j=1}^{\infty}$ be representable as integral linear combinations of elements of some Riesz basis; or that the matrix of transition from $\left\{x_{j}\right\}_{j=1}^{\infty}$ to some Riesz basis have integral entries and integral (not necessarily bounded) inverse matrix.

The author does not know any investigation in such an integral Riesz basis theory. Observe only the following fact: the system $\left\{j e_{j}\right\}_{j=1}^{\infty}$, where $\left\{e_{j}\right\}_{j=1}^{\infty}$ is an orthonormal basis in $H$, does not possess property $(\ddagger)$. Indeed, the norm of a linear combination of the kind $f=\sum_{j=1}^{\infty} a_{j} \cdot j e_{j}$ with $a_{j} \in \mathbb{Z}$ will be large if $\left\langle f, e_{j}\right\rangle \neq 0$ for some sufficiently large $j \in \mathbb{N}$.

Property $(\dagger)$ of a regular domain $\Omega$ is equivalent to property $(\ddagger)$ of the systems of homologies $\left\{\gamma_{j}\right\}_{j=1}^{\infty}$ in the space $H_{1, L^{2}}(\Omega)$ (or to property $(\ddagger)$ of the system of period reproducing kernels $\left\{\kappa_{j}\right\}_{j=1}^{\infty}$ in the space $H_{L^{2}}^{1}(\Omega)$ ). There exist regular domains without property $(\dagger)$. To check this, one may construct a domain $\Omega$ such that, first, the norms $\left\|\kappa_{j}\right\|_{\left(L_{c}^{2,1}(\Omega)\right)^{*}}$ tend to infinity sufficiently fast and, second, the scalar products $\left\langle\kappa_{j}, \kappa_{j^{\prime}}\right\rangle_{L_{c}^{2,1}(\Omega)}$ are small compared to the norms of kernels. These conditions can be ensured by consecutive cutting round holes $B_{j}$ from the disk $\mathbb{D}$ so that the quantities $\operatorname{diam}_{H} B_{j}$ tend to infinity sufficiently fast and the holes $B_{j}$ are sufficiently far from one another in the hyperbolic metric. One can prove that a domain constructed in this way will not possess property $(\dagger)$ : the system of kernels reproducing the periods around holes in such a domain is, in general, similar to the system $\left\{j e_{j}\right\}_{j=1}^{\infty}$ pointed out above. At the same time, the complete interpolation property of a regular domain implies its property $(\dagger)$ : an integral Riesz basis in $H_{1, L^{2}}(\Omega)$ is given by curves $\gamma_{j}$ linked to the holes $B_{j}$. Therefore, property $(\dagger)$ of the existence of an integral Riesz basis in Hilbert homologies is a nontrivial quasiconformal invariant of countably connected Riemann surfaces. 


\section{Appendix A. Some technical proofs}

A.1. Proof of Proposition 1.5. We prove the second assertion. Let the form $\omega \in$ $L_{c}^{2,1}(\Omega)$ be exact. For $t \in \mathbb{R}$, we have $\operatorname{Per}\left(\omega_{0}(a)+t \omega\right)=a$; the minimality of the norm of $\omega_{0}(a)$ implies that

$$
\left.\frac{d\left\|\omega_{0}(a)+t \omega\right\|_{L_{c}^{2,1}(\Omega)}^{2}}{d t}\right|_{t=0}=0 .
$$

Differentiation in accordance with (2) yields

$$
\int_{\Omega} \omega \wedge * \omega_{0}(a)=0
$$

which implies the second assertion.

Let $\eta \in W^{1,2}(\mathbb{D})$ be a function. Then $\left.d \eta\right|_{\Omega} \in L_{c}^{2,1}(\Omega)$. Since any such function $\eta$ can be approximated in $W^{1,2}(\mathbb{D})$ by smooth functions, we have $\operatorname{Per}_{j}(d \eta)=0$ for all $j=1,2, \ldots$. Therefore, by (40),

$$
\int_{\Omega} d \eta \wedge * \omega_{0}(a)=0
$$

for any $\eta \in W^{1,2}(\mathbb{D})$. Since the last relation is true for all $\eta \in C_{0}^{\infty}(\Omega)$, the form $* \omega_{0}$ is closed. In Subsection 2.2, with no use of the result that we are proving now, we shall construct functions $\mathfrak{v}_{j} \in \stackrel{\circ}{W}^{1,2}(\mathbb{D}), j=1,2, \ldots$, such that $\left\langle\omega,-\left(* d \mathfrak{v}_{j}\right)\right\rangle_{L_{c}^{2,1}}(\Omega)=\operatorname{Per}_{j} \omega$ for all $\omega \in L_{c}^{2,1}(\Omega)$. In our case $* \omega_{0} \in L_{c}^{2,1}(\Omega)$. Substituting $\eta=\mathfrak{v}_{j}$ in (41) gives $\operatorname{Per}_{j}\left(* \omega_{0}(a)\right)=0$ for all $j=1,2, \ldots$ Then the form $* \omega_{0}(a)$ is exact, that is, the form $\omega_{0}(a)$ is co-exact.

\section{A.2. Proofs from Subsection 2.2,}

Proof of Theorem 2.4. To prove the existence of the function $\mathfrak{v}_{j}$, it suffices to consider the closed affine subspace $Y \subset \stackrel{\circ}{W}^{1,2}(\mathbb{D})$ consisting of functions $u \in \stackrel{\circ}{W}^{1,2}(\mathbb{D})$ such that $u=1$ almost everywhere on $B_{j}$ and $u=0$ almost everywhere on $B_{j^{\prime}}$ for $j \neq j^{\prime}$. Observe that $Y \neq \varnothing$ because the holes $B_{j^{\prime}}$ do not accumulate to $B_{j}$ (this easily allows us to construct a function with the required boundary values and with finite Dirichlet integral). Now, as $\mathfrak{v}_{j}$ we may take an element with a minimal norm in $Y$. The uniqueness of a minimizing function follows from the fact that a sphere in a Hilbert space contains no segments. The identity $\Delta \mathfrak{v}_{j}=0$ in $\Omega$ is obtained by the usual variational argument.

The third assertion of the theorem follows from the second. Now we prove the second assertion. Let a function $v \in \stackrel{\circ}{W}^{1,2}(\mathbb{D})$ be a solution of the homogeneous boundary problem, that is, $v=0$ almost everywhere on each hole $B_{j}, j=1,2, \ldots$, and $\Delta v=0$ in $\Omega$. Then

$$
\int_{\Omega}\langle\nabla v, \nabla \eta\rangle d \lambda_{2}=0
$$

for any "test" function $\eta \in C_{0}^{\infty}(\Omega)$ (this is exactly the distributional form of the relation $\Delta v=0)$. Then, in order to prove that $v=0$, it suffices to approximate $v$ in $W^{1,2}(\mathbb{D})$ by smooth functions compactly supported in $\Omega$ (then (42) will imply that $\int_{\Omega}\langle\nabla v, \nabla v\rangle d \lambda_{2}=0$, whence $v=0$ almost everywhere in $\left.\mathbb{D}\right)$. For this, taking $\delta>0$ small, first we approximate $v$ by a function of the form $\psi_{\delta}(z) \cdot v(z)$, where $\psi_{\delta}: \mathbb{D} \rightarrow[0,1]$ is a smooth function, $\psi_{\delta}(z)=1$ for $|z| \in[0,1-2 \delta], \psi_{\delta}(z)=0$ for $|z| \in[1-\delta, 1]$, and 
$\left|\nabla \psi_{\delta}\right| \leq 2 / \delta$. We have $\nabla\left(\psi_{\delta} v\right)-\nabla v=\left(1-\psi_{\delta}\right) \nabla v+v \nabla \psi_{\delta}$, and

$$
\begin{aligned}
\left\|\psi_{\delta} v-v\right\|_{W^{1,2}(\mathbb{D})}^{2} & \leq 2 \int_{\mathbb{D}}\left(|\nabla v|^{2}\left(1-\psi_{\delta}\right)^{2}+v^{2}\left|\nabla \psi_{\delta}\right|^{2}\right) d \lambda_{2} \\
& \leq 2 \int_{|z| \geq 1-2 \delta}|\nabla v|^{2} d \lambda_{2}+8 \int_{|z| \geq 1-2 \delta} \frac{v^{2}(z) d \lambda_{2}(z)}{(1-|z|)^{2}}
\end{aligned}
$$

by the choice of $\psi_{\delta}$. The first integral in the last expression tends to zero as $\delta \rightarrow 0$ because $v \in \stackrel{\circ}{W}^{1,2}(\mathbb{D})$; the second integral also tends to zero as $\delta \rightarrow 0$ because, by Theorem 2.5.

$$
\int_{\mathbb{D}} \frac{v^{2}(z) d \lambda_{2}}{(1-|z|)^{2}} \leq \mathfrak{c} \cdot\|v\|_{W^{1,2}(\mathbb{D})}^{2}<+\infty .
$$

So, the functions $u_{\delta}=\psi_{\delta} v$ are supported in $\mathcal{B}(0,1-\delta)$ and tend to $v$ in $\stackrel{\circ}{W}^{1,2}(\mathbb{D})$ as $\delta \rightarrow 0$. Consider a domain $\Omega_{\delta} \subset \mathbb{D}$ containing $\mathcal{B}(0,1-\delta)$ and all the sets $B_{j^{\prime}}, j^{\prime}=1,2, \ldots$, that intersect the disk $\mathcal{B}(0,1-\delta)$. The domain $\Omega_{\delta}$ can be taken so that the boundary $\partial \Omega_{\delta}$ is smooth. Now $u_{\delta} \in \stackrel{\circ}{W}^{1,2}\left(\Omega_{\delta}\right)$ and $u=0$ on all the holes $B_{j^{\prime}}$; then $u_{\delta}$ can be approximated in $\stackrel{\circ}{W}^{1,2}\left(\Omega_{\delta}\right)$ by functions of class $C_{0}^{\infty}\left(\Omega_{\delta} \cap \Omega\right)$. Here we use the fact that the boundary $\partial\left(\Omega_{\delta} \cap \Omega\right)$ is smooth, which is true because all boundaries $\partial B_{j^{\prime}}$ are smooth. Hence, we can approximate $u_{\delta}$ in $\stackrel{\circ}{W}^{1,2}(\mathbb{D})$ by functions of class $C_{0}^{\infty}(\Omega)$, and therefore, $v$ also admits such approximation, which implies that $v=0$. The second assertion is proved.

Now we pass to the proof of the fourth assertion. First we check the case where $\Omega$ has a smooth boundary (and, in particular, a finite number of holes) and the form $\omega$ for which we want to prove (6) is sufficiently smooth. Namely, let

$$
\Omega=V \backslash \bigcup_{k=1}^{K} B_{k}
$$

where $V \subset \mathbb{C}$ is a domain with sufficiently smooth boundary, whereas the $B_{k}, k=$ $1,2, \ldots, K$, are disjoint compact subsets in $V$ also having smooth boundaries (the number of holes is finite). In this case it is well known (see, e.g., [7]) that the classical solution of the Dirichlet problem posed by analogy with problem (17) with the replacement of $\mathbb{D}$ by $V$, exists and is smooth up to the boundary $\partial \Omega$ (the problem is stated in the class $W^{1,2}(V)$ instead of $\left.\stackrel{\circ}{W}^{1,2}(\mathbb{D})\right)$. Let the form $\omega \in L_{c}^{2,1}(\Omega)$ be smooth up to the boundary $\partial \Omega$. Then

$$
\begin{aligned}
\left\langle\omega,-\left(* d \mathfrak{v}_{j}\right)\right\rangle_{L_{c}^{2,1}}(\Omega) & =\int_{\Omega} \omega \wedge\left(-\left(* * d \mathfrak{v}_{j}\right)\right) \\
& =\int_{\Omega} \omega \wedge d \mathfrak{v}_{j}=-\int_{\Omega} d\left(\mathfrak{v}_{j} \wedge \omega\right)=-\int_{\partial \Omega} \mathfrak{v}_{j} \omega
\end{aligned}
$$

(application of Stokes' theorem is justified due to the sufficient smoothness of the forms involved and of the boundaries of sets). Next, $\mathfrak{v}_{j}=0$ on $\partial \Omega \backslash \partial B_{j}$ and $\mathfrak{v}_{j}=1$ on $\partial B_{j}$, which implies that the last integral in (43) equals

$$
-\int_{\partial B_{j}} \omega=\operatorname{Per}_{j} \omega
$$

(again, due to the smoothness of the form $\omega$ up to $\partial \Omega$; the boundary $\partial B_{j}$ is oriented clockwise to be in accordance with the orientation of $\Omega$; but the periods are calculated as integrals in the counterclockwise direction, by our definition). Therefore, in the case of a domain $\Omega$ with sufficiently smooth boundary and a sufficiently smooth form $\omega$, identity (6) for $\kappa_{j}=-\left(* d \mathfrak{v}_{j}\right)$ is proved. 
Now we pass to the general case. Fix $j$. Let function $\mathfrak{v}_{j}$ be defined for the domain $\Omega$ as before, that is, by projection. We must prove that

$$
\left\langle\omega,-\left(* d \mathfrak{v}_{j}\right)\right\rangle_{L_{c}^{2,1}}(\Omega)=\operatorname{Per}_{j} \omega
$$

for any form $\omega \in L_{c}^{2,1}(\Omega)$. Observe that $\left\langle d v,-\left(* d \mathfrak{v}_{j}\right)\right\rangle_{L_{c}^{2,1}}(\Omega)=0$ for any function $v \in C_{0}^{\infty}(\Omega)$ : indeed, we have

$$
\left\langle d v,-\left(* d \mathfrak{v}_{j}\right)\right\rangle_{L_{c}^{2,1}}(\Omega)=\int_{\Omega} d v \wedge d \mathfrak{v}_{j}=\int_{\Omega} d\left(v \wedge d \mathfrak{v}_{j}\right)=0
$$

because $v$ is compactly supported. Therefore, $-\left(* d \mathfrak{v}_{j}\right) \perp \mathcal{F}_{1}(\Omega)$ (see (15)) in $L_{c}^{2,1}(\Omega)$, and moreover, $\left.\operatorname{Per}_{j}\right|_{\mathcal{F}_{1}(\Omega)} \equiv 0$. Then, by Proposition 2.1, it suffices to check (44) only for $\omega \in \operatorname{Harm}^{2,1}(\Omega)$. These forms are smooth up to the boundary in each strictly inner subdomain of $\Omega$ with sufficiently smooth boundary. We shall approximate $\Omega$ from below by such domains.

For $m=1,2, \ldots$, we choose domains $\Omega_{m} \subset \mathbb{D}$ such that:

(1) $\operatorname{clos} \Omega_{m} \subset \Omega_{m+1}, \bigcup_{m=1}^{\infty}=\Omega$;

(2) $\partial \Omega_{m} \in C^{\infty}$;

(3) the set $\Omega_{1}$ (and so all $\Omega_{m}, m=2,3, \ldots$ ) separates the hole $B_{j}$ from $\infty \in \hat{\mathbb{C}}$.

For any $m=1,2, \ldots$ the set $\mathbb{C} \backslash \Omega_{m}$ consists of only finitelly many connected components. Denote by $B_{j}^{(m)}$ such a component that includes $B_{j}$. Let $\mathfrak{v}_{j}^{(m)}$ be the solution of problem (77) for the set $\Omega_{m}$ (that is, $\Delta \mathfrak{v}_{j}^{(m)}=0$ in $\Omega_{m}$, and $\mathfrak{v}_{j}^{(m)}=1$ on $B_{j}^{(m)}, \mathfrak{v}_{j}^{(m)}=0$ on $\left.\partial \Omega_{j} \backslash \partial B_{j}^{(m)}\right)$. Defining $\mathfrak{v}_{j}^{(m)}$ to be 1 on $B_{j}^{(m)}$ and 0 on $\mathbb{D} \backslash\left(\Omega_{m} \cup B_{j}^{(m)}\right)$, we obtain a function of class $W^{1,2}(\mathbb{D})$.

The function $\mathfrak{v}_{j}^{(m)}: \mathbb{D} \rightarrow \mathbb{R}$ can be described as a function minimizing the Dirichlet integral $\|\mathfrak{v}\|_{W^{1,2}(\mathbb{D})}^{2}$ under the conditions $\left.\mathfrak{v}\right|_{B_{j}^{(m)}}=1$ almost everywhere, $\left.\mathfrak{v}\right|_{\mathbb{D} \backslash\left(\Omega_{m} \cup B_{j}^{(m)}\right)}=0$ almost everywhere. If $m$ increases then these conditions weaken, so that

$$
\left\|\mathfrak{v}_{j}^{(m)}\right\|_{\mathscr{W}^{1,2}(\mathbb{D})} \leq\left\|\mathfrak{v}_{j}^{(1)}\right\|_{\mathscr{W}^{1,2}(\mathbb{D})} .
$$

Thus, the sequence $\left\{\mathfrak{v}_{j}^{(m)}\right\}_{m=1}^{\infty}$ is bounded in $\mathscr{W}^{1,2}(\mathbb{D})$. In $\left\{\mathfrak{v}_{j}^{(m)}\right\}_{j=1}^{\infty}$ we choose a weakly convergent subsequence, namely, reserving the prior notation for the sequence, let us assume that $\mathfrak{v}_{j}^{(m)} \rightarrow \mathfrak{v}_{0}$ in $\stackrel{\circ}{W}^{1,2}(\mathbb{D})$ while $\nabla \mathfrak{v}_{j}^{(m)} \rightarrow \nabla \mathfrak{v}_{0}$ in $L^{2}(\mathbb{D})$ as $m \rightarrow \infty$, where $\mathfrak{v}_{0}$ is some function in $\stackrel{\circ}{W}^{1,2}(\mathbb{D})$.

Since $\mathfrak{v}_{j}^{(m)}=1$ almost everywhere on $B_{j}$ and $\mathfrak{v}_{j}^{(m)}=0$ almost everywhere on $B_{j^{\prime}}$ for $j^{\prime} \neq j$ (the domains $\Omega_{m}$ are smaller than $\Omega$ ), we have $\mathfrak{v}_{0}=1$ almost everywhere on $B_{j}$ and $\mathfrak{v}_{0}=0$ almost everywhere on $B_{j^{\prime}}$ for $j^{\prime} \neq j$ (the embeddings $\stackrel{\circ}{W}^{1,2}(\mathbb{D})$ in $L^{2}\left(B_{j}\right)$ and in $L^{2}\left(B_{j^{\prime}}\right)$ are compact). Since $\bigcup_{m=1}^{\infty} \Omega_{m}=\Omega$, we have $\Delta \mathfrak{v}_{0}=0$ in $\Omega$. The uniqueness of a solution of the Dirichlet problem (17) proved above implies that $\mathfrak{v}_{0}=\mathfrak{v}_{j}$.

Let us prove identity (44) for $\omega \in \operatorname{Harm}^{2,1}(\Omega)$. The form $\omega$ is smooth up to the boundary in each of the sets $\Omega_{m}, m=1,2, \ldots$ Thus, as it has already been proved,

$$
\operatorname{Per}_{j}(\omega)=\int_{\Omega_{m}} \omega \wedge-\left(* d \mathfrak{v}_{j}^{(m)}\right)
$$

We define the form $\omega$ to be zero on $\mathbb{D} \backslash \Omega$, then $\operatorname{Per}_{j}(\omega)=\int_{\mathbb{D}} \omega \wedge-\left(* d \mathfrak{v}_{j}^{(m)}\right)$ (the functions $\mathfrak{v}_{j}^{(m)}$ are defined and locally constant on $\left.\mathbb{D} \backslash \Omega_{m}\right)$. Passing to the weak limit in $L^{2}(\mathbb{D})$, we conclude that $\operatorname{Per}_{j} \omega=\int_{\mathbb{D}} \omega \wedge-\left(* d \mathfrak{v}_{j}\right)$. Thus, formula (44) is proved for harmonic forms, and it was mentioned above that then this relation is true for all forms $\omega \in L_{c}^{2,1}(\Omega)$. The theorem is proved completely. 
Proof of Proposition 2.6. Our goal is to apply the Stokes theorem to the integral

$$
\int_{\Omega}\left\langle\nabla \mathfrak{v}_{j}, \nabla \mathfrak{v}_{j^{\prime}}\right\rangle d \lambda_{2}
$$

for the functions $\mathfrak{v}_{j}$ and $\mathfrak{v}_{j^{\prime}}$ that we found in Theorem 2.4. To this end, we take a positive sequence $\left\{r_{m}\right\}_{m=1}^{\infty}$ of positive numbers increasing to 1 and such that the domains $\Omega_{m}:=\mathcal{B}\left(0, r_{m}\right) \cap \Omega$ have piecewise smooth boundaries (this can be done by Sard's lemma). Moreover, we may assume that $B_{j}, B_{j^{\prime}} \subset \Omega_{m}$. The functions $\mathfrak{v}_{j}, \mathfrak{v}_{j^{\prime}}$ are smooth up to $\partial \Omega_{m}$ (because they are harmonic in $\Omega$, see, e.g., [7] about the boundary smoothness of solutions of elliptic equations), and moreover, $\Delta \mathfrak{v}_{j^{\prime}}=0$ in $\Omega_{m}$, whence

$$
\int_{\Omega_{m}}\left\langle\nabla \mathfrak{v}_{j}, \nabla \mathfrak{v}_{j^{\prime}}\right\rangle d \lambda_{2}=\int_{\partial \Omega_{m}} \mathfrak{v}_{j} \cdot\left\langle\nabla \mathfrak{v}_{j^{\prime}}, \vec{n}\right\rangle d \mathcal{H}^{1},
$$

where $\vec{n}$ is the outward unit normal field on $\partial \Omega_{m}$ and $\mathcal{H}^{1}$ is the length measure. The set $\partial \Omega_{m}$ in the last integral is formed by $\Omega \cap \partial \mathcal{B}\left(0, r_{m}\right)$ and some pieces of the boundary of $\partial \Omega$. The contribution of the last pieces to the right-hand side of (45) equals $\int_{\partial B_{j}}\left\langle\nabla \mathfrak{v}_{j^{\prime}}, \vec{n}\right\rangle d \mathcal{H}^{1}$ because $\mathfrak{v}_{j}=0$ on $\partial B_{k}$ for $k \neq j$ and $\mathfrak{v}_{j}=1$ on $\partial B_{j}$. Moreover,

$$
\left|\int_{\Omega \cap \partial \mathcal{B}\left(0, r_{n}\right)} \mathfrak{v}_{j} \cdot\left\langle\nabla \mathfrak{v}_{j^{\prime}}, \vec{n}\right\rangle d \mathcal{H}^{1}\right| \leq\left(\int_{\partial \mathcal{B}\left(0, r_{n}\right)} \mathfrak{v}_{j}^{2} d \mathcal{H}^{1}\right)^{1 / 2}\left(\int_{\partial \mathcal{B}\left(0, r_{n}\right)}\left|\nabla \mathfrak{v}_{j^{\prime}}\right|^{2} d \mathcal{H}^{1}\right)^{1 / 2} .
$$

The last quantity can be made arbitrarily small by the choice of the sequence $r_{n}$ because $\mathfrak{v}_{j}, \mathfrak{v}_{j^{\prime}} \in \stackrel{\circ}{W}^{1,2}(\mathbb{D})$. Therefore,

$$
\int_{\Omega_{m}}\left\langle\nabla \mathfrak{v}_{j}, \nabla \mathfrak{v}_{j^{\prime}}\right\rangle d \lambda_{2} \stackrel{m \rightarrow \infty}{\longrightarrow} \int_{\partial B_{j}}\left\langle\nabla \mathfrak{v}_{j^{\prime}}, \vec{n}\right\rangle d \mathcal{H}^{1} .
$$

But $\int_{\Omega_{m}}\left\langle\nabla \mathfrak{v}_{j}, \nabla \mathfrak{v}_{j^{\prime}}\right\rangle d \lambda_{2} \stackrel{m \rightarrow \infty}{\longrightarrow}\left\langle\nabla \mathfrak{v}_{j}, \nabla \mathfrak{v}_{j^{\prime}}\right\rangle_{L^{2}(\mathbb{D})}=\left\langle\kappa_{j}, \kappa_{j^{\prime}}\right\rangle_{L_{c}^{2,1}(\Omega)}$ by the explicit form of the reproducing kernels $\kappa_{j}, \kappa_{j^{\prime}}$. So, $\left\langle\kappa_{j}, \kappa_{j^{\prime}}\right\rangle_{L_{c}^{2,1}(\Omega)}=\int_{\partial B_{j}}\left\langle\nabla \mathfrak{v}_{j^{\prime}}, \vec{n}\right\rangle d \mathcal{H}^{1}$.

The function $\mathfrak{v}_{j^{\prime}}$ was obtained as a solution of the Dirichlet boundary problem for the Laplace equation in $\Omega$ with the boundary values on $\partial \Omega$ equal to 0 or 1 ; the existence was proved by a variational argument. From this, it is not hard to derive that $\mathfrak{v}_{j^{\prime}} \geq 0$ in $\Omega$. This implies that $\left\langle\nabla \mathfrak{v}_{j^{\prime}}, \vec{n}\right\rangle \leq 0$ on $\partial B_{j}$ (because $\mathfrak{v}_{j^{\prime}}$ is smooth in $\Omega$ near $\partial B_{j}$ and equals zero on $\left.\partial B_{j}\right)$. On the other hand, the identity $\left\langle\nabla \mathfrak{v}_{j^{\prime}}, \vec{n}\right\rangle=0$ cannot be fulfilled everywhere on $\partial B_{j}$ due to the uniqueness of a solution of the Cauchy problem for the Laplace equation. Therefore, $\left\langle\kappa_{j}, \kappa_{j^{\prime}}\right\rangle_{L_{c}^{2,1}(\Omega)}=\int_{\partial B_{j}}\left\langle\nabla \mathfrak{v}_{j^{\prime}}, \vec{n}\right\rangle d \mathcal{H}^{1}<0$. The proof is finished.

\section{A.3. The other proofs in Section 2,}

Proof of Proposition 2.2. If $\omega \in \operatorname{Harm}^{2,1}(\Omega) \cap\left(L^{2,1}(\Omega) \ominus \mathcal{F}_{5}(\Omega)\right)$, then $\omega \in \mathcal{F}_{4}(\Omega)$ by the definition of the set $\mathcal{F}_{4}(\Omega)$ : indeed, the form $\omega$ is harmonic and is orthogonal to all the exact forms and hence to all the exact harmonic forms.

Now, let $\omega \in \mathcal{F}_{4}(\Omega)$. In order to prove that $\omega \in \operatorname{Harm}^{2,1}(\Omega) \cap\left(L^{2,1}(\Omega) \ominus \mathcal{F}_{5}(\Omega)\right)$, we must check that $\langle\omega, \eta\rangle_{L^{2,1}(\Omega)}=0$ for any exact form $\eta \in L^{2,1}(\Omega)$. We apply the decomposition (4) to the form $\eta$ :

$$
\eta=\eta_{1}+\eta_{2}+\eta_{3}, \quad \eta_{1} \in \mathcal{F}_{1}(\Omega), \eta_{2} \in \mathcal{F}_{2}(\Omega), \eta_{3} \in \operatorname{Harm}^{2,1}(\Omega) .
$$

Proposition 2.1 implies that $\eta_{2}=0$ (the form $\eta$ is exact and is then closed). Next, $\left\langle\omega, \eta_{1}\right\rangle_{L^{2,1}(\Omega)}=0$ by the orthogonality of the spaces $\mathcal{F}_{1}(\Omega)$ and $\operatorname{Harm}^{2,1}(\Omega)$. Finally, $\operatorname{Per}_{j}\left(\eta_{1}\right)=0$ for all $j=1,2, \ldots$ (because all periods of a form of the kind $d u, u \in$ $C_{0}^{\infty}(\Omega)$, are zero and the functional $\operatorname{Per}_{j}$ is continuous on $\left.L_{c}^{2,1}(\Omega)\right)$. On the other hand, $\operatorname{Per}_{j}(\eta)=0$ by the exactness of the form $\eta$, then $\operatorname{Per}_{j}\left(\eta_{3}\right)=0$ for any $j=1,2, \ldots$, and 
hence the form $\eta_{3}$ is exact. The condition $\omega \in \mathcal{F}_{4}(\Omega)$ implies that $\left\langle\omega, \eta_{3}\right\rangle_{L^{2,1}(\Omega)}=0$. Therefore, we have proved that $\langle\omega, \eta\rangle_{L^{2,1}(\Omega)}=0$, as required.

Proof of Proposition 2.13. To prove the first assertion it suffices to cut a function $u$ occurring under the infimum in (15) by the levels 0 and 1 . The second assertion is obvious.

To prove the third assertion, we take functions $u_{12}$ and $u_{13}$ from the definition (15) of the capacities $\operatorname{Cap}_{2}\left(E_{1}, E_{2}\right)$ and $\operatorname{Cap}_{2}\left(E_{1}, E_{3}\right)$ such that $u_{12}(z), u_{13}(z) \in[0,1]$ for almost all $z \in \mathbb{C}$. The function $u=\max \left\{u_{12}, u_{13}\right\}$ belongs to $W_{\text {loc }}^{1,2}(\mathbb{C})$ and can be put under the infimum in relation (15) defining $\operatorname{Cap}_{2}\left(E_{1}, E_{2} \cup E_{3}\right)$. Since $|\nabla u| \leq \max \left\{\left|\nabla u_{12}\right|,\left|\nabla u_{13}\right|\right\}$, we have $\int_{\mathbb{C}}|\nabla u|^{2} d \lambda_{2} \leq \int_{\mathbb{C}}\left|\nabla u_{12}\right|^{2} d \lambda_{2}+\int_{\mathbb{C}}\left|\nabla u_{13}\right|^{2} d \lambda_{2}$. It remains to pass to the infimum over $u_{12}$ and $u_{13}$.

Proof of Proposition 2.14. The second assertion can be proved by a simple push-forward of the functions $u$ in the definitions of capacities. We prove the first assertion. Let $\widetilde{u}$ be the function occurring in the definition of the capacity $\operatorname{Cap}_{2}\left(\varphi\left(E_{1}\right), \varphi\left(E_{2}\right)\right)$. Then we can find a cut-off function $\psi: \mathbb{C} \rightarrow[0,1]$ such that $\left.\psi\right|_{\mathbb{D}}=1, \psi \in C_{0}^{\infty}(\mathbb{C})$, and the norm $\|\nabla \widetilde{u}-\nabla(\psi \cdot \widetilde{u})\|_{L^{2}(\mathbb{C})}$ is arbitrarily small. Now it remains to plug the function $u=(\psi \widetilde{u}) \circ \varphi$ in the infimum defining $\operatorname{Cap}_{2}\left(E_{1}, E_{2}\right)$, make a change of variables, and pass to the infimum over $\widetilde{u}$.

Proof of Proposition 2.19. Put $T=\frac{1}{2} \min \left\{\operatorname{diam} E_{1}, \operatorname{diam} E_{2}\right\}$ and $\Lambda=\operatorname{dist}\left(E_{1}, E_{2}\right)$. Since the sets $E_{1}, E_{2}$ are closed and one of them is compact, there exist points $z_{1} \in$ $E_{1}, z_{2} \in E_{2}$ such that $\left|z_{1}-z_{2}\right|=\Lambda$. By using an orthogonal transformation of the plane, we may assume that $z_{1}=0, z_{2}=\Lambda$.

By the definition of capacity, there exists a function $u \in W_{\text {loc }}^{1,2}(\mathbb{C})$ such that $u \geq 1$ almost everywhere on $E_{1}, u \leq 0$ almost everywhere on $E_{2}$, and

$$
\int_{\mathbb{C}}|\nabla u|^{2} d \lambda_{2} \leq 2 \operatorname{Cap}_{2}\left(E_{1}, E_{2}\right) \text {. }
$$

Let $t \in[0, T)$. Consider a contour $\beta_{t}$ formed by two circles $\partial \mathcal{B}(0, t)$ and $\partial \mathcal{B}(\Lambda, t)$ and two line segments $[0, \Lambda]+i t$ and $[0, \Lambda]-i t$. Repairing, if necessary, the function $u$ on a set of zero measure, we may assume that $u$ is absolutely continuous on the contour $\beta_{t}$ for almost all $t \in(0, T)$. The circle $\partial \mathcal{B}(0, t)$ must have nonempty intersection with Int $E_{1}$, whereas $\partial \mathcal{B}(\Lambda, t)$ intersects Int $E_{2}$, because $0 \in E_{1}, \Lambda \in E_{2}$, the diameters of the sets $E_{1}$ and $E_{2}$ are larger than $2 t$, and these sets are connected. Therefore, $\mathcal{H}^{1}\left(\beta_{t} \cap E_{1}\right), \mathcal{H}^{1}\left(\beta_{t} \cap E_{2}\right)>0$. Hence, for almost all $t \in(0, T)$ the function $u$ must have some values not less than 1 and some values not exceeding 0 on the contour $\beta_{t}$. The length of this contour $\beta_{t}$ is $4 \pi t+2 \Lambda$ and this contour is connected. Then, by the Cauchy-Schwartz inequality,

$$
\int_{\beta_{t}}|\nabla u|^{2} d \mathcal{H}^{1} \geq \frac{1}{4 \pi t+2 \Lambda}
$$

for almost all $t \in(0, T)$. Each point $z \in \mathbb{C}$ may belong to $\beta_{t}$ for at most three values of $t$. Integration of the estimate obtained over $t \in(0, T)$ gives

$$
3 \int_{\mathbb{C}}|\nabla u|^{2} d \lambda_{2} \geq \int_{0}^{T} \frac{d t}{4 \pi t+2 \Lambda}=\frac{1}{4 \pi} \log \left(1+\pi \cdot \frac{2 T}{\Lambda}\right) .
$$

Now the desired estimate follows from the inequality $\int_{\mathbb{C}}|\nabla u|^{2} d \lambda_{2} \leq 2 \operatorname{Cap}_{2}\left(E_{1}, E_{2}\right)$.

Proof of Proposition 2.21. Let $j, k=1,2, \ldots, j \neq k$. Let $\operatorname{diam} B_{j} \geq \operatorname{diam} B_{k}$. By the second assertion of Proposition 2.20, $\operatorname{dist}\left(B_{j}, B_{k}\right) \geq \varepsilon_{0} \cdot \operatorname{diam} B_{k}$, where $\varepsilon_{0}>0$ depends only on $\widetilde{C}_{B}(\Omega)$. We prove that $\operatorname{dist}\left(B_{j}, B_{k}\right) \geq \varepsilon \cdot \operatorname{diam} B_{j}$ with some $\varepsilon$ depending only on the constants from the hypothesis. $\operatorname{Since} \operatorname{diam}_{H}\left(B_{k}\right) \geq r$, it follows that $\operatorname{dist}\left(B_{k}, \partial \mathbb{D}\right) \leq$ 
$c_{1} \cdot \operatorname{diam} B_{k}$, where $c_{1}$ depends only on $r$ (the first assertion of Proposition 2.17). By the second assertion of the same proposition and also by the first assertion of Proposition 2.20. we have $\operatorname{dist}\left(B_{j}, \partial \mathbb{D}\right) \geq c_{2} \cdot \operatorname{diam} B_{j}$, where $c_{2}>0$ depends only on $\widetilde{C}_{B}(\Omega)$. Suppose that $\operatorname{dist}\left(B_{j}, B_{k}\right) \leq \frac{c_{2}}{2} \cdot \operatorname{diam} B_{j}$. Then

$$
\begin{aligned}
c_{2} \operatorname{diam} B_{j} \leq \operatorname{dist}\left(B_{j}, \partial \mathbb{D}\right) & \leq \operatorname{dist}\left(B_{j}, B_{k}\right)+\operatorname{diam} B_{k}+\operatorname{dist}\left(B_{k}, \partial \mathbb{D}\right) \\
& \leq \frac{c_{2}}{2} \cdot \operatorname{diam} B_{j}+\left(1+c_{1}\right) \operatorname{diam} B_{k},
\end{aligned}
$$

which yields

$$
\operatorname{diam} B_{j} \leq \frac{2\left(1+c_{1}\right)}{c_{2}} \cdot \operatorname{diam} B_{k}
$$

Now the estimate for $\operatorname{diam}_{H}\left(B_{k}\right)$ shows that

$$
\operatorname{dist}\left(B_{j}, B_{k}\right) \geq \varepsilon_{0} \cdot \operatorname{diam} B_{k} \geq \frac{c_{2} \varepsilon_{0}}{2\left(1+c_{1}\right)} \cdot \operatorname{diam} B_{j} .
$$

So, we may take $\min \left\{\frac{c_{2}}{2}, \frac{c_{2} \varepsilon_{0}}{2\left(1+c_{1}\right)}\right\}$ for the role of $\varepsilon$, this quantity is positive and depends only on $r$ and $\widetilde{C}_{B}(\Omega)$.

Proof of Proposition 2.22. Consider the function

$$
u(z):=\frac{\operatorname{dist}(z, \mathbb{C} \backslash U)}{\Lambda \varepsilon}, \quad z \in \mathbb{C} .
$$

Then $u \geq 1$ on $E$ and $u=0$ on $\mathbb{C} \backslash U$. Also, the function $u$ is Lipschitz and, moreover,

$$
|\nabla u| \leq \frac{1}{\Lambda \varepsilon}
$$

almost everywhere; $\nabla u=0$ outside of $U$. The area of $U$ is at most $\pi(1+2 \varepsilon)^{2} \Lambda^{2}$ because its diameter does not exceed $(1+2 \varepsilon) \Lambda$. Hence,

$$
\int_{\mathbb{R}^{2}}|\nabla u|^{2} d \lambda_{2} \leq \frac{\pi(1+2 \varepsilon)^{2}}{\varepsilon^{2}} .
$$

Substituting the function $u$ in the infimum from the definition of capacity gives the result.

Proof of Proposition 2.24. The condition

$$
\sup _{j \in \mathbb{N}} \operatorname{diam}_{H}\left(B_{j}\right)<+\infty
$$

implies that $\operatorname{dist}\left(B_{j}, \partial \mathbb{D}\right) \geq \varepsilon_{1} \cdot \operatorname{diam} B_{j}$ for each $j=1,2, \ldots$, where $\varepsilon_{1}>0$ depends only on $\sup _{j \in \mathbb{N}} \operatorname{diam}_{H}\left(B_{j}\right)$. Put $\varepsilon_{0}:=\min \left\{\varepsilon_{1} / 3, \varepsilon / 3\right\}$, this constant is positive and depends only on $\varepsilon$ and $\sup _{j \in \mathbb{N}} \operatorname{diam}_{H}\left(B_{j}\right)$. Put $\Lambda_{j}=\operatorname{diam} B_{j}, j=1,2, \ldots$ By the definition of the strong separation property, the sets $U_{\varepsilon_{0} \Lambda_{j}}\left(B_{j}\right)$ are pairwise disjoint; moreover, $U_{\varepsilon_{0} \Lambda_{j}}\left(B_{j}\right) \subset \mathbb{D}$ for all $j=1,2, \ldots$

Let $u_{j}, j=1,2, \ldots$, be the functions constructed in the proof of Proposition 2.22 for set $B_{j}$ and the complement to its neighborhood $U_{\varepsilon_{0} \Lambda_{j}}\left(B_{j}\right)$, that is, $u_{j}=1$ almost everywhere on $B_{j}$ and $u_{j}=0$ almost everywhere outside of $U_{\varepsilon_{0} \Lambda_{j}}\left(B_{j}\right),\left\|u_{j}\right\|_{W^{1,2}(\mathbb{D})} \leq C$, here $C$ depends only on $\varepsilon_{0}$ and thus only on the constants from the hypothesis.

To prove the Bessel property of the domain $\Omega$, we check the assumptions of the first assertion of Proposition 2.10. Let $a=\left\{a_{j}\right\}_{j=1}^{\infty} \in \ell^{2}$. Put $u_{a}:=\sum_{j=1}^{\infty} a_{j} u_{j}$. For such finite sums we have $\left\|u_{a}\right\|_{W^{1,2}(\mathbb{D})}^{2} \leq C^{2} \cdot\|a\|_{\ell^{2}}$ (the sets supp $u_{j}$ are pairwise disjoint). Hence, the series $\sum_{j=1}^{\infty} a_{j} u_{j}$ is convergent in $\stackrel{\circ}{W}^{1,2}(\mathbb{D})$ for any sequence $a=\left\{a_{j}\right\}_{j=1}^{\infty} \in \ell^{2}$, 
and $\left\|u_{a}\right\|_{\mathscr{W}^{1,2}(\mathbb{D})} \leq C \cdot\|a\|_{\ell^{2}}$. The sum $u_{a}$ of this series is an admissible function because each $u_{j}$ is admissible. Also $\left.u_{a}\right|_{B_{j}} \equiv a_{j}$ almost everywhere. Therefore, the assumptions of Proposition 2.10 are fulfilled, whence $\Omega$ possesses the Bessel property with $C_{B}(\Omega) \leq C$.

A.4. Proof of Lemma 5.3 on partial order. We start with the choice of the constant $c_{1}$. Since $\sup _{j \in \mathbb{N}} \operatorname{diam}_{H}\left(B_{j}\right)<+\infty$, it follows that there exists a constant $c_{h}>0$ such that $U_{c_{h} \Lambda_{j}}\left(B_{j}\right) \subset \mathbb{D}$ for all $j=1,2, \ldots$ (see Proposition 2.17). We may assume that $c_{1}<c_{h} / 2$. It is easy to show that in this case $\operatorname{dist}_{H}\left(z, B_{j}\right) \leq 2$ for any point $z \in U_{c_{1} \Lambda_{j}}\left(B_{j}\right)$; therefore, $\operatorname{diam}_{H} U_{c_{1} \Lambda_{j}}\left(B_{j}\right) \leq \operatorname{diam}_{H} B_{j}+4$. We also assume that $c_{1}<\varepsilon / 2$, where $\varepsilon$ is the constant of the weak separation of the holes $B_{j}$. The choice of $c_{1}$ will be refined below.

The uniform local finiteness property implies the existence of $M \in \mathbb{N}$ such that any set of hyperbolic diameter not exceeding $4+\sup _{j \in \mathbb{N}} \operatorname{diam}_{H}\left(B_{j}\right)$ intersects at most $M$ holes $B_{j}\left(M=N\left(\Omega, 4+\sup _{j \in \mathbb{N}} \operatorname{diam}_{H} B_{j}\right)\right)$.

Lemma A.1. Under the assumptions of Theorem [5.1, there exists a constant $C_{2}=$ $C_{2}(\varepsilon, M)<+\infty$ such that for any $j=1,2, \ldots$ and any positive $c_{1}<\min \left\{c_{h} / 2, \varepsilon / 2\right\}$ there exists an open interval $I \subset\left(0, c_{1} \Lambda_{j}\right)$ with length at least $c_{1} \Lambda_{j} / C_{2}(\varepsilon, M)$ for which

$$
\left\{z \in \mathbb{R}^{2}: \operatorname{dist}\left(z, B_{j}\right) \in I\right\} \subset \Omega .
$$

Proof. Fix $j$. Let $B_{j_{1}}, B_{j_{2}}, \ldots, B_{j_{m}}$ be the holes intersecting $U_{c_{1} \Lambda_{j}}\left(B_{j}\right)$ (except for the hole $B_{j}$ ). Their number $m$ does not exceed $M$ by the choice of $c_{1}<c_{h} / 2$. Let $\left[t_{k}, s_{k}\right]$ be the image of the set $B_{j_{k}}$ under the mapping $\operatorname{dist}\left(\cdot, B_{j}\right)(k=1,2, \ldots, m)$. This is indeed a segment because $B_{j_{k}}$ is a continuum.

By the choice of $c_{1}$, we have $\operatorname{dist}\left(B_{j}, B_{j_{k}}\right)<\varepsilon \cdot \operatorname{diam} B_{j}$ for all $k=1,2, \ldots, m$. But the holes in $\Omega$ are $\varepsilon$-weakly separated. Therefore, $\operatorname{dist}\left(B_{j}, B_{j_{k}}\right) \geq \varepsilon \operatorname{diam} B_{j_{k}}$. This implies that

$$
\frac{s_{k}-t_{k}}{t_{k}} \leq \frac{1}{\varepsilon}
$$

We are going to show that the last estimate and the boundedness of $m$ imply that $\left[0, c_{1} \Lambda_{j}\right]$ contains an interval $I$ with $\lambda_{1}(I) \geq c_{2} \Lambda_{j}$ that does not intersect $\left[t_{k}, s_{k}\right]$; here $c_{2}>0$ is not very small. Take $c_{2}<c_{1}$ and suppose the contrary. We may assume that $t_{1} \leq t_{2} \leq \cdots \leq t_{m}$. If $t_{1}>c_{2} \Lambda_{j}$, then we may take an interval of the form $I=\left[\delta, \delta+c_{2} \Lambda_{j}\right]$ with $\delta>0$ sufficiently small. Therefore, $t_{1} \leq c_{2} \Lambda_{j}$ and from (46) we have $s_{1} \leq(1+1 / \varepsilon) c_{2} \Lambda_{j}$. Let $k=2,3, \ldots, m$. If $t_{k}>c_{2} \Lambda_{j}+\max \left\{s_{1}, s_{2}, \ldots, s_{k-1}\right\}$, then, again, we may take $I=\max \left\{s_{1}, s_{2}, \ldots, s_{k-1}\right\}+\delta+\left[0, c_{2} \Lambda_{j}\right]$ with small positive $\delta$. Thus,

$$
\begin{aligned}
t_{k} & \leq \max \left\{s_{1}, s_{2}, \ldots, s_{k-1}\right\}+c_{2} \Lambda_{j}, \\
s_{k} & \leq\left(1+\frac{1}{\varepsilon}\right) t_{k} .
\end{aligned}
$$

Consecutive application of these estimates for $k=2,3, \ldots, m$ gives an inequality of the form $s_{k} \leq C(\varepsilon, k) \cdot c_{2} \Lambda_{j}$, where $C(\varepsilon, k) \in(0,+\infty)$ depends only on $k$ and $\varepsilon$. Put $C_{2}(\varepsilon, M):=2 \max _{k \leq M} C(\varepsilon, k)+3$ and take $c_{2}:=c_{1} / C_{2}(\varepsilon, M)$. If, on $\left[0, c_{1} \Lambda_{j}\right]$, between the intervals $\left[t_{k}, s_{k}\right]$ and also to the left of them there is no unoccupied interval of length $c_{2} \Lambda_{j}$, then $s_{k}<c_{1} \Lambda_{j} / 2$ for all $k=1,2, \ldots, m$. Now for the role of $I$ we may take an interval of length $c_{2} \Lambda_{j}$ in $\left[c_{1} \Lambda_{j} / 2, c_{1} \Lambda_{j}\right)$.

Now we continue the proof of Lemma 5.3, Let us refine the choice of $c_{1}$ : in addition to the restrictions imposed above, let us assume that $c_{1} \cdot\left(1+C_{2}(\varepsilon, M)\right)<\varepsilon$, where $\varepsilon$ is the 
constant of weak separation of the holes, whereas the number $C_{2}(\varepsilon, M)$ was constructed in Lemma A.1. Put $c_{2}:=c_{1} / C_{2}(\varepsilon, M)$. In particular, we have

$$
c_{1}^{2} / c_{2}+c_{1}<\varepsilon
$$

Denote by $I=\left[t_{j}, s_{j}\right]$ the interval constructed in the preceding lemma for the hole $B_{j}$, $j=1,2, \ldots$ Put $A_{j}:=U_{s_{j}}\left(B_{j}\right) \backslash U_{t_{j}}\left(B_{j}\right)$. The sets $A_{j}$ satisfy all the requirements in the first assertion of the lemma (the inclusion $A_{j} \subset \Omega$ is true by the choice of $\left[t_{j}, s_{j}\right]$ ). We check the second assertion. Let $z \in A_{j_{1}} \cap A_{j_{2}} \cap \cdots \cap A_{j_{m}}$ for some indices $j_{1}, j_{2}, \ldots, j_{m}$. We estimate $m$. As it was mentioned before Lemma A.1, then we have $\operatorname{dist}_{H}\left(z, B_{j_{k}}\right) \leq 2$ for all $k=1,2, \ldots, m$. Consider a ball in the hyperbolic metric of radius 2 centered at $z$. This ball intersects all the sets $B_{j_{k}}, k=1,2, \ldots, m$. Hence, $m \leq N(\Omega, 2)$ by the definition of the constant $N(\Omega, 2)$. We have checked the second assertion of the lemma.

Note that in general, the set $A_{j}$ may turn out to be disconnected even if $B_{j}$ is connected and simply connected. (It suffices to take the difference of two neighborhoods of a set $B_{j}$ looking like an arc of a circle of some radius $r$, with the length of this arc close to $2 \pi r$.) Even if the set $U_{s_{k}}\left(B_{j}\right) \backslash U_{t_{k}}\left(B_{j}\right)$ is connected, it may fail to be homeomorphic to an annulus. Our construction simplifies if the holes are disks: then the $A_{j}$ are annuli. However, the mutual placement of even round annuli may happen to be complicated. Namely, it may turn out that $A_{j} \cap A_{k} \neq \varnothing$ for some $j, k=1,2, \ldots$, but $U_{s_{j}}\left(B_{j}\right) \not \subset U_{s_{k}}\left(B_{k}\right)$ and $U_{s_{k}}\left(B_{k}\right) \not \subset U_{s_{j}}\left(B_{j}\right)$. We need to show that our choice of $c_{1}$ excludes such a possibility.

The relation $\prec$ of strict partial order on the set of holes $B_{j}$ is defined as follows: $B_{j^{\prime}} \prec B_{j}$ if and only if $j^{\prime} \neq j$ and $U_{s_{j}^{\prime}}\left(B_{j^{\prime}}\right) \subset U_{s_{j}}\left(B_{j}\right)$. By the definition, this relation is transitive and antireflexive.

We check the remaining part of the third assertion of the lemma. Let $B_{j^{\prime}} \prec B_{j}$. Then $j^{\prime} \neq j$ and $B_{j^{\prime}} \subset U_{s_{j^{\prime}}}\left(B_{j^{\prime}}\right) \subset U_{s_{j}}\left(B_{j}\right)$ by the definition of the order $\prec$. Now we check that if $B_{j^{\prime}} \subset U_{s_{j}}\left(B_{j}\right)$ and $j^{\prime} \neq j$, then $B_{j^{\prime}} \prec B_{j}$. We need to prove that $U_{s_{j^{\prime}}}\left(B_{j^{\prime}}\right) \subset U_{s_{j}}\left(B_{j}\right)$. The set $A_{j}=U_{s_{j}}\left(B_{j}\right) \backslash U_{t_{j}}\left(B_{j}\right)$ lies in $\Omega$ and thus does not intersect the hole $B_{j^{\prime}}$. Therefore, $B_{j^{\prime}} \subset U_{t_{j}}\left(B_{j}\right)$, in particular, $\operatorname{dist}\left(B_{j}, B_{j^{\prime}}\right) \leq t_{j}$. By the choice of $t_{j}$ and by the $\varepsilon$-weak separation of the holes we have the inequality

$$
c_{1} \Lambda_{j} \geq t_{j} \geq \operatorname{dist}\left(B_{j}, B_{j^{\prime}}\right) \geq \min \left\{\varepsilon \Lambda_{j}, \varepsilon \Lambda_{j^{\prime}}\right\} .
$$

The choice of $c_{1}$ shows that $c_{1} \Lambda_{j}<\varepsilon \Lambda_{j}$, which implies $\Lambda_{j^{\prime}}<\Lambda_{j}$ and $c_{1} \Lambda_{j} \geq \varepsilon \Lambda_{j^{\prime}}$, i.e., $\Lambda_{j^{\prime}} \leq c_{1} \Lambda_{j} / \varepsilon$. By the choice of $s_{j^{\prime}}$ we have $s_{j^{\prime}} \leq c_{1} \Lambda_{j^{\prime}} \leq c_{1}^{2} \Lambda_{j} / \varepsilon$. By the choice of $c_{1}$, the last quantity is strictly smaller than $c_{2} \Lambda_{j}$. Therefore, $s_{j^{\prime}}+t_{j}<c_{2} \Lambda_{j}+t_{j} \leq s_{j}$. We conclude that $U_{s_{j^{\prime}}}\left(B_{j^{\prime}}\right) \subset U_{s_{j^{\prime}}+t_{j}}\left(B_{j}\right) \subset U_{s_{j}}\left(B_{j}\right)$. The third assertion of Lemma [5.3 is proved.

Now we prove the fourth assertion in Lemma 5.3. For a fixed $j=1,2, \ldots$, we estimate the number of holes $B_{j^{\prime}}$ for which $B_{j^{\prime}} \prec B_{j}$. For such $j^{\prime}$ we have $B_{j^{\prime}} \subset U_{t_{j}}\left(B_{j}\right)$. But, as it has been observed above, $\operatorname{diam}_{H}\left(U_{t_{j}}\left(B_{j}\right)\right) \leq 4+\sup _{j \in \mathbb{N}} \operatorname{diam}_{H}\left(B_{j}\right)$. Thus, there exist at most $N\left(\Omega, 4+\sup _{j \in \mathbb{N}} \operatorname{diam}_{H}\left(B_{j}\right)\right)$ indices $j^{\prime}$ such that $B_{j^{\prime}} \prec B_{j}$. This implies that the lengths of the chains in the order $\prec$ are finite and can be estimated from above in terms of constants from the hypothesis of the theorem only.

Now we check that if $B_{j_{1}}, B_{j_{2}} \succ B_{j}$, then either $B_{j_{1}} \succ B_{j_{2}}$, or $B_{j_{2}} \succ B_{j_{1}}$. (In other words, $\left\{B_{j^{\prime}}: B_{j^{\prime}} \succ B_{j}\right\}$ is a chain in the order $\succ$.) We may assume that $j_{1}=1$ and $j_{2}=2$. Since $B_{j} \subset U_{t_{1}}\left(B_{1}\right) \cap U_{t_{2}}\left(B_{2}\right)$ (by the third assertion of the lemma), we have $U_{t_{1}}\left(B_{1}\right) \cap U_{t_{2}}\left(B_{2}\right) \neq \varnothing$. Therefore, in particular,

$$
\operatorname{dist}\left(B_{1}, B_{2}\right) \leq t_{1}+t_{2} \leq c_{1}\left(\Lambda_{1}+\Lambda_{2}\right) .
$$

There is no loss of generality in assuming that $\Lambda_{2} \leq \Lambda_{1}$. From the $\varepsilon$-weak separation condition of for the sets $B_{1}$ and $B_{2}$ we have $\operatorname{dist}\left(B_{1}, B_{2}\right) \geq \varepsilon \Lambda_{2}$ (because $c_{1}<\varepsilon / 2$ ). 
Taking (48) into account, we obtain $\varepsilon \Lambda_{2} \leq c_{1}\left(\Lambda_{1}+\Lambda_{2}\right)$, so that

$$
\Lambda_{1} \geq \frac{\varepsilon-c_{1}}{c_{1}} \Lambda_{2}
$$

If $B_{2} \nprec B_{1}$ then, by the third assertion of the lemma, $B_{2} \not \subset U_{t_{1}}\left(B_{1}\right)$. The distance between the sets $U_{t_{1}}\left(B_{1}\right)$ and $\mathbb{R}^{2} \backslash U_{s_{1}}\left(B_{1}\right)$ is positive, whereas $B_{2}$ is connected and does not intersect $A_{1}=U_{s_{1}}\left(B_{1}\right) \backslash U_{t_{1}}\left(B_{1}\right)$. Therefore, $\operatorname{dist}\left(B_{2}, B_{1}\right) \geq s_{1} \geq t_{1}+c_{2} \Lambda_{1}$ by the choice of $t_{1}$ and $s_{1}$. Comparing this with (48), we have $c_{2} \Lambda_{1} \leq t_{2} \leq c_{1} \Lambda_{2}$ (the last inequality is true by the choice of $t_{2}$ ), whence $\Lambda_{1} \leq c_{1} \Lambda_{2} / c_{2}$. This inequality and (49) imply

$$
\frac{\varepsilon-c_{1}}{c_{1}} \leq \frac{c_{1}}{c_{2}},
$$

or $\varepsilon \leq c_{1}^{2} / c_{2}+c_{1}$. But this contradicts inequality (47) established in the course of the choice of $c_{1}$. Thus, we have checked all the properties necessary for our construction. Lemma 5.3 is proved.

\section{A.5. Proofs from Subsection 6.2,}

Proof of Lemma 6.1. The monotonicity required in all the three assertions is immediate from Corollary 2.11.

For any nicely increasing sequence of domains, we want to justify the limit passage from the third assertion. For this, we take any sequence $a \in \ell^{2}$ and observe that, for each $m=1,2, \ldots$, there exists a form $\omega_{m} \in L_{c}^{2,1}\left(\Omega_{m}\right)$ with $\operatorname{Per}^{\left(\Omega_{m}\right)} \omega_{m}=a,\left\|\omega_{m}\right\|_{L_{c}^{2,1}\left(\Omega_{m}\right)} \leq$ $C_{I}\left(\Omega_{m}\right) \cdot\|a\|_{\ell^{2}}$. Define $\omega_{m}$ to be zero in $\mathbb{D} \backslash \Omega_{m}$. In $\left\{\omega_{m}\right\}_{m=1}^{\infty}$ we can select a subsequence weakly* convergent in $L^{2}(\mathbb{D})$, obtaining a form $\omega \in L_{c}^{2,1}(\Omega)$ as a weak limit, for which $\operatorname{Per}^{(\Omega)} \omega=a,\|\omega\|_{L_{c}^{2,1}(\Omega)} \leq\|a\|_{\ell^{2}} \cdot \lim \sup _{m \rightarrow \infty} C_{I}\left(\Omega_{m}\right)$. This implies that $C_{I}(\Omega) \leq$ $\limsup _{m \rightarrow \infty} C_{I}\left(\Omega_{m}\right)$, and the monotonicity of the interpolation constants gives us the third assertion.

The limit passage for the Bessel and the weak Bessel constants requires additional considerations.

Lemma A.2. Let $\Omega$ be a domain with arbitrary holes, let $B_{1}, B_{2}, \ldots$ be bounded connected components of $\Omega^{(c)}$, and let $\lambda>1$. There exist sets $B_{1}^{\prime}, B_{1}^{\prime \prime}, B_{2}^{\prime}, B_{2}^{\prime \prime}, \cdots \subset \mathbb{D}$ such that:

(1) for any $j=1,2, \ldots$ the sets $B_{j}^{\prime}, B_{j}^{\prime \prime}$ are simply connected closures of domains with smooth boundaries; $B_{j} \subset \operatorname{Int} B_{j}^{\prime} \subset B_{j}^{\prime} \subset \operatorname{Int} B_{j}^{\prime \prime} \subset B_{j}^{\prime \prime}$; the sets $B_{j}^{\prime \prime}$ are pairwise disjoint and accumulate only to $\partial \mathbb{D}$ as $j \rightarrow \infty$;

(2) there exists a quasiconformal diffeomorphism $\varphi$ of $\Omega$ onto the domain $\Omega^{\prime}:=$ $\mathbb{D} \backslash \bigcup_{j=1}^{\infty} B_{j}^{\prime}$ for which $K(\varphi) \leq \lambda^{2}$; moreover, $\varphi(z)=z$ if $z \in \Omega \backslash \bigcup_{j=1}^{\infty} B_{j}^{\prime \prime}$.

Proof. We do this by a step-by-step process. Suppose that, at the $(j-1)$ st step $(j=$ $1,2, \ldots)$, we have already constructed sets $B_{1}^{\prime}, B_{1}^{\prime \prime}, B_{2}^{\prime}, B_{2}^{\prime \prime}, \ldots, B_{j-1}^{\prime}, B_{j-1}^{\prime \prime}$, each not intersecting $B_{j}$. Let us construct sets $B_{j}^{\prime}$ and $B_{j}^{\prime \prime}$. Let $\psi_{j}$ be a conformal bijection of the set $(\mathbb{C} \cup\{\infty\}) \backslash B_{j}$ onto $(\mathbb{C} \cup\{\infty\}) \backslash$ clos $\mathbb{D}$. Such a mapping exists because $B_{j}$ is connected, does not separate the plane and consists of more than one point (see [3]). We may assume that $\psi_{j}(\infty)=\infty$.

The holes $B_{j+1}, B_{j+2}, \ldots$ do not accumulate to $B_{1}$. Since the mapping $\psi_{j}$ is open, we deduce easily that the set $\psi_{j}\left(\mathbb{D} \backslash\left(B_{1}^{\prime \prime} \cup B_{2}^{\prime \prime} \cup \cdots \cup B_{j-1}^{\prime \prime} \cup B_{j} \cup B_{j+1} \cup \ldots\right)\right)$ contains an annulus of the form $\overline{\mathcal{B}}\left(0, r_{j}\right) \backslash \operatorname{clos} \mathbb{D}$ with some $r_{j}>1$.

Pick $\rho_{j} \in\left(1, r_{j}\right)$. Assume that $\rho_{j}$ is so close to 1 that there exists a quasiconformal diffeomorphism $\widetilde{\varphi}_{j}$ from $(\operatorname{clos} \mathbb{D})^{(c)}$ onto $\mathbb{C} \backslash \overline{\mathcal{B}}\left(0, \rho_{j}\right)$ such that $K\left(\widetilde{\varphi}_{j}\right) \leq \lambda^{2}$ and $\widetilde{\varphi}_{j}(z)=z$ for $|z| \geq r_{j}$. 
The sets $\psi_{j}^{-1}\left(\partial \mathcal{B}\left(0, \rho_{j}\right)\right)$ and $\psi_{j}^{-1}\left(\partial \mathcal{B}\left(0, r_{j}\right)\right)$ are smooth Jordan curves on the plane and even in the disk $\mathbb{D}$. By the Jordan theorem, each of them encloses a closed bounded set; we take the first of these sets as $B_{j}^{\prime}$, and the second as $B_{j}^{\prime \prime}$. Since $\psi_{j}(\infty)=\infty$ and the circle $\partial \mathcal{B}\left(0, r_{j}\right)$ separates $\infty$ from $\partial \mathcal{B}\left(0, \rho_{j}\right)$, we have $B_{j}^{\prime} \subset B_{j}^{\prime \prime}$ (and, thus, $\left.B_{j}^{\prime} \subset \operatorname{Int} B_{j}^{\prime \prime}\right)$. The circle $\partial \mathcal{B}\left(0, r_{j}\right)$ does not separate the sets $\psi_{j}\left(B_{k}^{\prime \prime}\right)$ for $k=1,2, \ldots, j-1$ and the sets $\psi_{j}\left(B_{l}\right)$ for $l=j+1, j+2, \ldots$ from $\infty$; hence, $B_{j}^{\prime \prime}$ does not intersect $B_{k}^{\prime \prime}$ and $B_{l}$ for such $k$ and $l$. The last fact allows us to continue the process of constructing the sets $B_{l}^{\prime \prime}$. The set $\psi_{j}^{-1}\left(\mathcal{B}\left(0, \rho_{j}\right) \backslash\right.$ clos $\left.\mathbb{D}\right)$ cannot be simply connected; this implies that $B_{j} \subset \operatorname{Int} B_{j}^{\prime}$.

We also need to provide the last assertion of the first statement of the lemma, i.e., to act in such a way that the sets $B_{j}^{\prime \prime}$ do accumulate only to $\partial \mathbb{D}$. For that, it suffices to perform our construction so that the set $B_{j}^{\prime \prime}$ lie in the 1-neighborhood of the set $B_{j}$ in the hyperbolic metric. Since $B_{j} \subset \mathbb{D}$, it suffices to make $B_{j}^{\prime \prime}$ lie in the $\varepsilon$-neighborhood of $B_{j}$ in the Euclidean metric for some $\varepsilon>0$ depending on $B_{j}$; it suffices to check the last condition only for the curve $\psi_{j}^{-1}\left(\partial \mathcal{B}\left(0, r_{j}\right)\right)$. Since the mapping $\psi_{j}$ is open, it is easily seen that this can be done by shrinking, if necessary, the radius $r_{j}$ (before choosing $\rho_{j}$ ).

Performing such steps for all $j=1,2, \ldots$ gives us sets required in the first assertion of the lemma. Let us construct the mapping $\varphi$ occurring in the second assertion. For this, put $\varphi(z)=z$ if $z$ does not belong to the union of the constructed sets $B_{j}^{\prime \prime}, j=1,2, \ldots$ If $z \in B_{j}^{\prime \prime} \backslash B_{j}$ for some $j$, then we put $\varphi(z):=\left(\psi_{j}^{-1} \circ \tilde{\varphi}_{j} \circ \psi_{j}\right)(z)$. Then $\varphi$ is a smooth mapping without critical points in the entire set $\Omega$ by the construction of the mappings $\tilde{\varphi}_{j}$. From this and the fact that the mappings $\psi_{j}$ is conformal we have the estimate $K(\varphi) \leq \lambda^{2}$. Furthermore, the annulus $\mathcal{B}\left(0, r_{j}\right) \backslash \overline{\mathcal{B}}\left(0, \rho_{j}\right)$ consists of points separated from $\infty$ by the curve $\partial \mathcal{B}\left(0, r_{j}\right)$, but not by the curve $\partial \overline{\mathcal{B}}\left(0, \rho_{j}\right)$. Therefore, $\psi^{-1}\left(\mathcal{B}\left(0, r_{j}\right) \backslash \overline{\mathcal{B}}\left(0, \rho_{j}\right)\right)=\left(\operatorname{Int} B_{j}^{\prime \prime}\right) \backslash B_{j}^{\prime}$. We see that $\varphi$ is a diffeomorphism of $\Omega$ onto $\mathbb{D} \backslash \bigcup_{j=1}^{\infty} B_{j}^{\prime}$. The second assertion of the lemma is proved. The proof is complete.

Now we finish the proof of Lemma 6.1 For $m=1,2, \ldots$ we apply Lemma A.2 to the domain $\Omega$ and $\lambda=1+1 / m$. Let $B_{j, m}^{\prime}, B_{j, m}^{\prime \prime}, j=1,2, \ldots$, be the holes with smooth boundaries constructed in that lemma, and let $\Omega_{m}^{\prime}=\mathbb{D} \backslash \bigcup_{j=1}^{\infty} B_{j, m}^{\prime}$. Denote by $\varphi_{m}$ the corresponding mappings from the second assertion of the same lemma. Then $\varphi_{m}$ is a diffeomorphism of $\Omega$ onto $\Omega_{m}^{\prime}$. For any $j=1,2, \ldots$, the mapping $\varphi_{m}$ carries a curve in $\Omega$ winding around the hole $B_{j}$ onto a curve in the domain $\Omega_{m}^{\prime}$ winding around $B_{j, m}^{\prime}$. Indeed, by the first assertion of Lemma A.2 we may take a contour lying in $\mathbb{D} \backslash \bigcup_{j=1}^{\infty} B_{j, m}^{\prime \prime}$ as such a curve, but on this contour the mapping $\varphi_{m}$ is identical. It has been mentioned that Proposition 1.3 stays true for domains with arbitrary holes. Therefore, $C_{B}\left(\Omega_{m}^{\prime}\right) \leq$ $(1+1 / m) \cdot C_{B}(\Omega), \widetilde{C}_{B}\left(\Omega_{m}^{\prime}\right) \leq(1+1 / m) \cdot \widetilde{C}_{B}(\Omega)$. So, $\lim \sup _{m \rightarrow \infty} C_{B}\left(\Omega_{m}^{\prime}\right) \leq C_{B}(\Omega)$, $\lim \sup _{m \rightarrow \infty} \widetilde{C}_{B}\left(\Omega_{m}^{\prime}\right) \leq \widetilde{C}_{B}(\Omega)$.

It remains to provide the monotonicity of the resulting sequence of domains. By the monotonicity of the Bessel, weak Bessel, and interpolation constants with respect to domains, this can be done by shrinking the domains $\Omega_{m}^{\prime}$ in such an way that the sequence of domains obtained will decrease. But this condition can be ensured by a consecutive choice of domains $\Omega_{1}^{\prime \prime}, \Omega_{2}^{\prime \prime}, \ldots$ so that for every $m=1,2, \ldots$ the domain $\Omega_{m}$ stay regular, lie inside $\Omega_{m}^{\prime}$ and inside the already constructed $\Omega_{1}^{\prime \prime}, \Omega_{2}^{\prime \prime}, \ldots, \Omega_{m-1}^{\prime \prime}$, and still contain $\Omega$. Lemma 6.1 is proved.

Proof of Proposition 6.6. The holes in $\Omega$ accumulate only to $\partial \mathbb{D}$. Therefore, for each $j=1,2, \ldots$ there exists $\delta>0$ such that the $\delta$-neighborhood of the hole $B_{j}$ in the Euclidean metric intersects only finitely many other holes. This observation allows us to construct a sequence of open sets $U_{j}, j=1,2, \ldots$, such that $B_{j} \subset U_{j}$ for all $j$ 
and the sets $\operatorname{clos} U_{j}$ are $\varepsilon / 2$-weakly separated. Also, it is easy to ensure the estimate $\sup _{j \in \mathbb{N}} \operatorname{diam}_{H}\left(U_{j}\right)<\sup _{j \in \mathbb{N}} \operatorname{diam}_{H}\left(B_{j}\right)+1$ for all $j=1,2, \ldots$

For each $j=1,2, \ldots$, choose a set $B_{j}^{\prime}$ with smooth boundary such that $B_{j} \subset B_{j}^{\prime} \subset$ $U_{j}$. The domain $\Omega^{\prime}:=\mathbb{D} \backslash \bigcup_{j=1}^{\infty} B_{j}^{\prime}$ is regular. By construction, the holes in $\Omega_{m}^{\prime}$ are $\varepsilon / 2$-weakly separated, and $\sup _{j \in \mathbb{N}} \operatorname{diam}_{H}\left(B_{j, m}\right) \leq \sup _{j \in \mathbb{N}} \operatorname{diam}_{H}\left(B_{j}\right)+1$. We may also assume that $B_{j}^{\prime}$ lies in the 1-neighborhood of the set $B_{j}$ in the hyperbolic metric. Then $N\left(\Omega_{m}\right) \leq N(\Omega, 2)$. Theorem 5.1 implies that the constant $C_{B}\left(\Omega^{\prime}\right)$ is finite and can be estimated from above in terms of $\varepsilon, \sup _{j \in \mathbb{N}} \operatorname{diam}_{H}\left(B_{j}\right)$, and $N(\Omega)$ only. But $C_{B}(\Omega)$ does not exceed $C_{B}\left(\Omega^{\prime}\right)$ and, thus, is finite and admits an estimate in terms of the constants from the hypothesis.

Proof of Proposition 6.7. Let $S^{\prime}$ and $M^{\prime}$ be the constants whose existence is provided by Theorem 5.4 for domains $\Omega^{\prime}$ such that $C_{I}\left(\Omega^{\prime}\right) \leq C_{I}(\Omega)$ and $N\left(\Omega^{\prime}\right) \leq N(\Omega, 2)$.

Let $\alpha>0$ be such that if $E \subset \mathbb{D}$ and $\operatorname{diam}_{H} E \leq \alpha$, then we have the inequality $\operatorname{dist}(E, \partial \mathbb{D}) \geq 2 S^{\prime} \cdot \operatorname{diam} E$. Denote by $J$ the set of indices of the holes $B_{j}$ in the domain $\Omega$ for which $\operatorname{diam}_{H}\left(B_{j}\right) \leq \alpha / 2$. Choose a number $S \geq S^{\prime}$ such that if $E \subset \mathbb{D}$ and $\operatorname{diam}_{H} E \geq \alpha / 2$, then $\operatorname{dist}(E, \partial \mathbb{D}) \leq S \cdot \operatorname{diam} E$. Put $M=M^{\prime}+1$. The numbers $S$ and $M$ depend only on the constants from the hypothesis of our proposition. We prove our claim with such $S$ and $M$.

Choose a sequence of regular domains $\Omega_{m}, m=1,2, \ldots$, nicely increasing to $\Omega$, for which the estimates of Lemma 6.1 are true. These domains can be chosen so that if $B_{j, m}$ is one of the holes in $\Omega_{m}$ and $B_{j}$ is the hole in $\Omega$ corresponding to $B_{j, m}$, then $\operatorname{diam}_{H}\left(B_{j, m}\right) \leq 2 \operatorname{diam}_{H}\left(B_{j}\right)$ and $B_{j, m}$ lies in the 1-neighborhood of $B_{j}$ in the hyperbolic metric. Then $N\left(\Omega_{m}\right) \leq N(\Omega, 2)$. Moreover, $C_{I}\left(\Omega_{m}\right) \leq C_{I}(\Omega)$ by the monotonicity of the interpolation constant. Therefore, for each $m=1,2, \ldots$ the graph $G\left(\Omega_{m}, S^{\prime}\right)$ is connected, and $\operatorname{dist}_{G\left(\Omega_{m}, S^{\prime}\right)}\left(B_{j, m}, \mathbb{D}^{(c)}\right) \leq M$ for all $j \in \mathbb{N}$. We identify the corresponding vertices of these graphs as this was done before Proposition 6.7 if some edge occurs in all the graphs $G\left(\Omega_{m}, S^{\prime}\right)$, then it occurs also in the graph $G\left(\Omega, S^{\prime}\right)$.

If $j_{0} \in J$, then the degree of the vertex $B_{j_{0}, 1}$ in the graph $G\left(\Omega_{1}, S^{\prime}\right)$ is finite. Indeed, for any such $j_{0}$ we have $\operatorname{diam}_{H}\left(B_{j_{0}, 1}\right) \leq \alpha$ by construction. If a vertex $B_{j, 1}$ is adjacent in the graph $G\left(\Omega_{1}, S^{\prime}\right)$ to the vertex $B_{j_{0}, 1}$, then

$$
\operatorname{dist}\left(B_{j_{0}, 1}, B_{j, 1}\right) \leq S^{\prime} \cdot \operatorname{diam} B_{j_{0}, 1} \leq \operatorname{dist}\left(B_{j_{0}, 1}, \partial \mathbb{D}\right) / 2 .
$$

But the Euclidean $\left(\operatorname{dist}\left(B_{j_{0}, 1}, \partial \mathbb{D}\right) / 2\right)$-neighborhood of the set $B_{j_{0}, 1}$ can intersect only a finite number of other holes in $\Omega_{1}$, because the closure of that neighborhood lies strictly inside the disk $\mathbb{D}$, whereas the domain $\Omega_{1}$ is regular.

Let $j_{0} \in J$. Let $\mathcal{T}_{m}$ denote the set of all paths $\tau$ in the graph $G\left(\Omega_{m}, S^{\prime}\right)$ such that:

(1) $\tau$ has edge length at most $M^{\prime}$;

(2) $\tau$ starts at the vertex $B_{j_{0}, m}$;

(3) all the vertices of the path $\tau$, except for its end, are of the form $B_{j, m}$ with some $j \in J$

(4) the end of the path $\tau$ is either $\mathbb{D}^{(c)}$, or one of the holes $B_{j^{\prime}, m}$ with $j^{\prime} \notin J$.

By the monotonicity of the sequence $\left\{\Omega_{m}\right\}_{m=1}^{\infty}$, the set of edges of the graph $G\left(\Omega_{m}, S^{\prime}\right)$ decreases if $m$ increases. Then $\mathcal{T}_{m}$ decreases with the growth of $m$. Next, the degrees of the vertices $B_{j, 1}$ in the graph $G\left(\Omega_{1}, S\right)$ are finite for $j \in J$; then the set $\mathcal{T}_{1}$ is finite. Since the distance from $B_{j_{0}, m}$ to $\mathbb{D}^{(c)}$ in the graph $G\left(\Omega_{m}, S^{\prime}\right)$ does not exceed $M^{\prime}$, the sets $\mathcal{T}_{m}$ are nonempty for all $m=1,2, \ldots$ Therefore, there exists a path $\tau$ belonging to $\mathcal{T}_{m}$ for all $m=1,2, \ldots$. From the definition of the graphs $G\left(\Omega_{m}, S^{\prime}\right)$ and $G\left(\Omega, S^{\prime}\right)$, it follows that all edges of the path $\tau$ occur also in the graph $G\left(\Omega, S^{\prime}\right)$, whereas the length of $\tau$ does not exceed $M^{\prime}$. The last vertex of the path $\tau$ in the graph $G\left(\Omega, S^{\prime}\right)$ is either 
$\mathbb{D}^{(c)}$ or a hole $B_{j}$ such that $\operatorname{diam}_{H} B_{j} \geq \alpha / 2$. By the choice of $S$, in the second case such a vertex is adjacent to $\mathbb{D}^{(c)}$ in the graph $G(\Omega, S)$. But $\tau$ is a path also in the graph $G(\Omega, S)$. Therefore, the vertex $B_{j_{0}}$ can be joined in the graph $G(\Omega, S)$ with the vertex $\mathbb{D}^{(c)}$ by a path of edge length not exceeding $M^{\prime}+1$. The same is true also for the vertices of the form $B_{j^{\prime}}$ with $j^{\prime} \notin J^{\prime}$, because these vertices are adjacent in $G(\Omega, S)$ to $\mathbb{D}^{(c)}$ by the choice of $S$. The proof is complete.

\section{REFERENCES}

[1] L. V. Ahlfors, Lectures on quasiconformal mappings, D. Van Nostrend Co., Princeton, NJ, 1966. MR.0200442

[2] N. K. Bari, Biorthogonal systems and basis in Hilbert space, Moskov. Gos. Univ. Uchen. Zap. Mat. 148 (1951), 69-107. (Russian) MR0050171

[3] G. Goluzin, Geometric theory of functions of a complex variable, Nauka, Moscow, 1966; English transl., Transl. Math. Monogr., vol. 26, Amer. Math. Soc., Providence, RI, 1969. MR0247039

[4] G. de Rham, Varietes differentiables. Formes, courants, formes harmoniques, Herman, Paris, 1955. MR 0068889

[5] S. Katok, Fuchsian groups, Chicago Lectures Math., Univ. Chicago Press, Chicago, IL, 1992. MR.1177168

[6] G. H. Hardy, J. E. Littlewood, and G. Pólya, Inequalities, 2nd ed., Cambridge Univ. Press, Cambridge, 1952. MR 0046395

[7] O. A. Ladyzhenskaya and N. N. Ural'tseva, Linear and quasilinear elliptic equations, Nauka, Moscow, 1964; English transl., Acad. Press, New York-London, 1968. MR0244627

[8] V. G. Maz'ya, Sobolev spaces, Leningrad. Univ., Leningrad, 1985; English transl., Springer Ser. Soviet Math., Springer-Verlag, Berlin, 1985. MR.817985

[9] E. V. Malinnikova and V. P. Khavin, Uniform approximation by harmonic differential forms. A constructive approach, Algebra i Analiz 9 (1997), no. 6, 156-196; English transl., St. Petersburg Math. J. 9 (1998), no. 6, 1149-1180. MR1610176

[10] N. K. Nikol'skiü, Treatise on the shift operator, Nauka, Moscow, 1980; English transl., Grundlehren Math. Wiss., Bd. 273, Springer-Verlag, Berlin, 1986. MR827223

[11] A. Presa Sage and V. P. Khavin, Uniform approximation by harmonic differential forms in Euclidean space, Algebra i Analiz 7 (1995), no. 6, 104-152; English transl., St. Petersburg Math. J. 7 (1996), no. 6, 943-977. MR 1381980

[12] R. D. M. Accola, Differentials and extremal length on Riemann surfaces, Proc. Nat. Acad. Sci. USA 46 (1960), no. 4, 540-543. MR0118829

[13] H. Yamaguchi, Equilibrium vector potentials in $\mathbb{R}^{3}$, Hokkaido Math. J. 25 (1996), no. 1, 1-53. MR 1376490

[14] B. Fuglede, Extremal length and functional completion, Acta Math. 98 (1957), no. 3, $171-218$. MR 0097720

[15] , Extremal length and closed extensions of partial differential operators, Jul. Giellerups Boghandel, Copenhagen, 1960. MR0168887

[16] K. Seip, Interpolation and sampling in spaces of analytic functions, Univ. Lecture Ser., vol. 33, Amer. Math. Soc., Providence, RI, 2004. MR2040080

[17] G. Schwarz, Hodge decomposition - a method for solving boundary value problems, Lecture Notes in Math., vol. 1607, Springer-Verlag, Berlin, 1995. MR.1367287

Chebyshev Laboratory, St. Petersburg State University, 14th Line 29B, Vasilyevsky Island, SAint Petersburg 199178, Russia

E-mail address: mikhail.dubashinskiy@gmail.com

Received 27/NOV/2015

Translated by THE AUTHOR 